\title{
The symplectic geometry of higher Auslander algebras: Symmetric products of disks
}

\author{
Tobias Dyckerhoff ${ }^{1}$, Gustavo Jasso ${ }^{2}$ and Yank $\iota$ Lekili $^{3}$ \\ ${ }^{1}$ Fachbereich Mathematik, Universität Hamburg, Bundesstraße 55, 20146 Hamburg, Germany; \\ E-mail: tobias.dyckerhoff@uni-hamburg.de. \\ ${ }^{2}$ Mathematisches Institut, Rheinische Friedrich-Wilhelms-Universität Bonn, Endenicher Allee 60, 53115 Bonn, Germany; \\ E-mail: gjasso@math.uni-bonn.de. \\ ${ }^{3}$ Department of Mathematics, King's College London, Strand, London WC2R 2LS, United Kingdom; \\ E-mail: yanki.lekili@kcl.ac.uk.
}

Received: 28 June 2020; Revised: 29 November 2020; Accepted: 17 December 2020

2020 Mathematics subject classification: Primary - 16G70; Secondary - 19D99; 53D37

\begin{abstract}
We show that the perfect derived categories of Iyama's $d$-dimensional Auslander algebras of type $\mathbb{A}$ are equivalent to the partially wrapped Fukaya categories of the $d$-fold symmetric product of the 2-dimensional unit disk with finitely many stops on its boundary. Furthermore, we observe that Koszul duality provides an equivalence between the partially wrapped Fukaya categories associated to the $d$-fold symmetric product of the disk and those of its $(n-d)$-fold symmetric product; this observation leads to a symplectic proof of a theorem of Beckert concerning the derived Morita equivalence between the corresponding higher Auslander algebras of type $\mathbb{A}$. As a by-product of our results, we deduce that the partially wrapped Fukaya categories associated to the $d$-fold symmetric product of the disk organise into a paracyclic object equivalent to the $d$-dimensional Waldhausen $\mathrm{S}_{\bullet}$-construction, a simplicial space whose geometric realisation provides the $d$-fold delooping of the connective algebraic $K$-theory space of the ring of coefficients.
\end{abstract}

\section{Introduction}

Let $n$ and $d$ be natural numbers and consider the poset

$$
\left\{\begin{array}{l}
\mathbf{n} \\
d
\end{array}\right\}=\left\{I \in \mathbb{N}^{d} \mid 1 \leq i_{1} \leq i_{2} \leq \cdots \leq i_{d} \leq n\right\}
$$

of $d$-element multisubsets of $\mathbf{n}=\{1, \ldots, n\}$, where $I \leq J$ if for each $1 \leq a \leq d$ the inequality $i_{a} \leq j_{a}$ is satisfied. Further, introduce the subset $\left\{\begin{array}{l}\mathbf{n} \\ d\end{array}\right\}^{b} \subseteq\left\{\begin{array}{l}\mathbf{n} \\ d\end{array}\right\}$ consisting of those $I \in\left\{\begin{array}{l}\mathbf{n} \\ d\end{array}\right\}$ such that there exists an index $1 \leq a<d$ with $i_{a}=i_{a+1}$. Thus, the complement $\left(\begin{array}{l}\mathbf{n} \\ d\end{array}\right)=\left\{\begin{array}{l}\mathbf{n} \\ d\end{array}\right\} \backslash\left\{\begin{array}{l}\mathbf{n} \\ d\end{array}\right\}^{b}$ can be identified with the set of $d$-element subsets of $\mathbf{n}$ equipped with the natural product order. Equivalently, $\left\{\begin{array}{l}\mathbf{n} \\ d\end{array}\right\}$ is described as the poset of monotone functions

$$
\{1<2<\cdots<d\} \longrightarrow\{1<2<\cdots<n\}
$$

equipped with the usual partial order; under this identification, $\left(\begin{array}{l}\mathbf{n} \\ d\end{array}\right) \subset\left\{\begin{array}{l}\mathbf{n} \\ d\end{array}\right\}$ is the subposet spanned by the injective monotone functions. 
Let $\mathbf{k}$ be a field and define the finite-dimensional $\mathbf{k}$-algebra

$$
A_{n, d}:=\left(\bigoplus_{I \leq J} \mathbf{k} f_{J I}\right) /\left\langle f_{K K} \mid K \in\left\{\begin{array}{l}
\mathbf{n} \\
d
\end{array}\right\}^{b}\right\rangle
$$

equipped with the multiplication law

$$
f_{K J^{\prime}} \cdot f_{J I}= \begin{cases}f_{K I} & \text { if } J=J^{\prime} \\ 0 & \text { otherwise }\end{cases}
$$

Equivalently, $A_{n, d}$ is the quotient of the incidence k-algebra of the poset $\left\{\begin{array}{l}\mathbf{n} \\ d\end{array}\right\}$ by its two-sided ideal generated by the idempotents $f_{K K}, K \in\left\{\begin{array}{l}\mathbf{n} \\ d\end{array}\right\}^{\text {b }}$ (note that the $\mathbf{k}$-algebra $A_{n, d}$ vanishes if $n<d$ and is isomorphic to the base field $\mathbf{k}$ if $n=d$ ). For example, an $A_{n, 1}$-module corresponds to a $\mathbf{k}$-vector-spacevalued representation

$$
V_{1} \rightarrow V_{2} \rightarrow \cdots \rightarrow V_{n}
$$

of the linearly oriented $A_{n}$-quiver, while an $A_{n, 2}$-module amounts to a commutative diagram

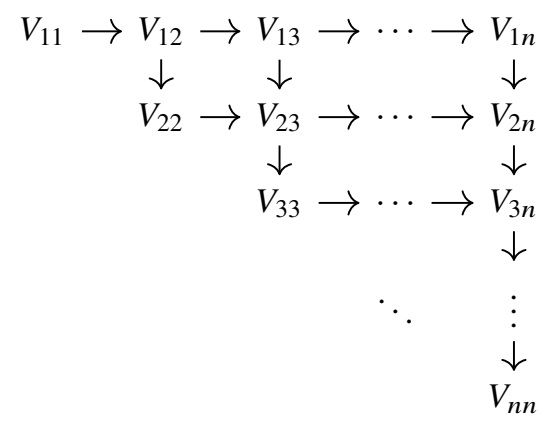

of $\mathbf{k}$-vector spaces with vanishing diagonal terms $V_{a a}, 1 \leq a \leq n$.

Remarkably, the algebras $A_{n, d}$ arise naturally in three a priori unrelated contexts within representation theory, algebraic $K$-theory, and symplectic topology:

(A) Higher Auslander-Reiten theory. The classical Auslander correspondence [Aus71], one of the cornerstones of the representation theory of finite-dimensional algebras, establishes a bijection

$$
\begin{gathered}
\left\{\begin{array}{c}
\text { finite-dimensional k-algebras } \\
\text { of finite representation type }
\end{array}\right\} \\
\uparrow \\
\left\{\begin{array}{c}
\text { finite-dimensional k-algebras } \Gamma \text { with } \\
\text { gl. dim } \Gamma \leq 2 \leq \text { dom. } \operatorname{dim} \Gamma
\end{array}\right\}
\end{gathered}
$$

where both classes of algebras are considered up to Morita equivalence; we remind the reader that dom. $\operatorname{dim} \Gamma$, the dominant dimension in the sense of [Tac64], is the largest number $d$ such that, in a minimal injective coresolution

$$
0 \rightarrow \Gamma_{\Gamma} \rightarrow I^{0} \rightarrow I^{1} \rightarrow \cdots \rightarrow I^{d-1} \rightarrow I^{d} \rightarrow \cdots
$$

of the regular representation $\Gamma_{\Gamma}$, the injective $\Gamma$-modules $I^{0}, I^{1}, \ldots, I^{d-1}$ are also projective. The correspondence is realised by associating to a k-algebra $A$ of finite representation type its Auslander algebra

$$
\Gamma_{A}=\operatorname{End}_{A}\left(\oplus_{[M]} M\right),
$$


where the sum ranges over the isomorphism classes of indecomposable $A$-modules $M$. This correspondence relates representation-theoretic properties of $A$ to homological properties of $\Gamma$.

For example, the k-algebra $A_{n+1,2}$ is the Auslander algebra corresponding to the k-algebra $A_{n, 1}$. As shown in [Iya11], for $n \geq d>2$ the algebra $A_{n, d}$ instead satisfies the inequalities

$$
\text { gl. } \operatorname{dim} \Gamma \leq d \leq \text { dom. } \operatorname{dim} \Gamma \text {. }
$$

Thus, $A_{n, d}$ belongs to the class of $d$-dimensional Auslander algebras introduced by Iyama in [Iya07a] as central objects of study in a higher-dimensional version of Auslander-Reiten theory. From now on we will refer to the k-algebras $A_{n, d}$ as the higher Auslander algebras of Dynkin type $\mathbb{A}$. Due to their rich combinatorial structure, this family of algebras has garnered quite some attention in representation theory - see, for example, [HI11, IO11, OT12, HIO14, GI19, DJW19], where they are mostly referred to as ' $d$-representation finite algebras of type $\mathbb{A}$ ', as well as the closely related [IO13, JK19].

(B) Waldhausen $K$-theory. By a construction of Waldhausen [BGT13, Wal85], the sequence

$$
n \longmapsto \operatorname{perf}\left(A_{n, 1}\right)
$$

of perfect derived (DG-)categories of the k-algebras $A_{n, 1}, n \geq 0$, organises into a simplicial differential graded k-category which provides a model for the Waldhausen $K$-theory [Wal85] of the field $\mathbf{k}$. More precisely, the Waldhausen $K$-theory space

$$
K(\mathbf{k})=\Omega\left|N_{\mathrm{dg}}\left(\operatorname{perf}\left(A_{\bullet, 1}\right)\right)^{\simeq}\right|
$$

of $\mathbf{k}$ is defined to be the 1-fold loop space of the geometric realisation of the simplicial $\infty$ groupoid $N_{\mathrm{dg}}\left(\operatorname{perf}\left(A_{\bullet}, 1\right)\right)^{\simeq}$ (obtained by passing to the largest Kan complex level-wise), leading to the formula $K_{i}(\mathbf{k}) \cong \pi_{i}(K(\mathbf{k}))$ for the higher algebraic $K$-groups of $\mathbf{k}$ previously defined by Quillen [Qui73].

For $d>1$, as discussed for abelian categories in the work of Poguntke [Pog17] and in [Dyc17b, DJW19] in the stable context, the sequence

$$
n \longmapsto \operatorname{perf}\left(A_{n, d}\right)
$$

of perfect derived (DG-)categories also forms a simplicial category. The relation to Waldhausen $K$-theory is then given by the formula

$$
K(\mathbf{k}) \simeq \Omega^{d} \mid N_{\mathrm{dg}}\left(\operatorname{perf}\left(A_{\bullet}, d\right)\right) \simeq
$$

so that for a fixed natural number $d$, the simplicial relations among the algebras $A_{n, d}$ encode the $d$-fold delooping of the $K$-theory space $K(\mathbf{k})$.

(C) Wrapped Floer theory. Let $\mathbb{D} \subset \mathbb{C}$ be the closed unit disk. For definiteness, fix the subset $\Lambda_{n} \subset \partial \mathbb{D}$ of $(n+1)$ th roots of unity. It is well known that there is a quasi-equivalence of $A_{\infty}$-categories

$$
\operatorname{perf}\left(A_{n, 1}\right) \stackrel{\simeq}{\longrightarrow} \mathcal{W}\left(\mathbb{D}, \Lambda_{n}\right)
$$

where $\mathcal{W}\left(\mathbb{D}, \Lambda_{n}\right)$ denotes the partially wrapped Fukaya category [Aur10a, HKK17] of $\mathbb{D}$ with stops in $\Lambda_{n}$. The main result of the present article, stated later, establishes a higher-dimensional version of this equivalence, providing a symplectic interpretation of all higher Auslander algebras of Dynkin type $\mathbb{A}$. 


\subsection{Our results}

Let $\mathbf{k}$ be a commutative ring. The following is the main theorem of this article:

Theorem 1 (Theorem 3.2.3). Let $\mathbb{D} \subset \mathbb{C}$ be the closed unit disk and let $\Lambda_{n} \subset \partial \mathbb{D}$ be the subset of $(n+1)$ th roots of unity. Then there is a quasi-equivalence of triangulated $A_{\infty}$-categories

$$
\operatorname{perf}\left(A_{n, d}\right) \stackrel{\simeq}{\longrightarrow} \mathcal{W}\left(\operatorname{Sym}^{d}(\mathbb{D}), \Lambda_{n}^{(d)}\right)
$$

between the perfect derived $A_{\infty}$-category of the $\mathbf{k}$-algebra $A_{n, d}$ and the $\mathbb{Z}$-graded partially wrapped Fukaya category of the dth symmetric power $\operatorname{Sym}^{d}(\mathbb{D})$ of $\mathbb{D}$ with stops

$$
\Lambda_{n}^{(d)}=\bigcup_{p \in \Lambda_{n}} p+\operatorname{Sym}^{d-1}(\mathbb{D}) .
$$

It is worth noting that when working over a field, the global dimension of the k-algebra $A_{n, d}$, which is $d$ provided that $n>d$, is reflected in the dimension of the symplectic manifold $\operatorname{Sym}^{d}(\mathbb{D})$, which is a real $2 d$-dimensional ball.

The proof of Theorem 1 relies on an explicit computation of the derived endomorphism algebra $\mathcal{A}_{n, d}$ of a specific set of generators of the $A_{\infty}$-category $\mathcal{W}\left(\operatorname{Sym}^{d}(\mathbb{D}), \Lambda_{n}^{(d)}\right)$. As it turns out, $\mathcal{A}_{n, d}$ is in fact a formal differential graded k-algebra whose (degree 0) cohomology is isomorphic to $A_{n, d}$ as an ungraded k-algebra (in the parlance of representation theory, we construct an explicit tilting object in $\mathcal{W}\left(\operatorname{Sym}^{d}(\mathbb{D}), \Lambda_{n}^{(d)}\right)$ whose endomorphism algebra is isomorphic to $\left.A_{n, d}\right)$. The differential graded kalgebra $\mathcal{A}_{n, d}$ was originally introduced in the context of bordered Heegaard Floer homology [LOT18] under the name 'strands algebra with $d$ strands and $n$ places'; its cohomology is computed in [LOT15, Section 4.1]. However, those authors work in characteristic 2. For this reason we follow an approach (hinted at in [LOT18, LOT15]) which relates the strands algebra to the Bruhat order on the symmetric group on $d$ letters.

The connection between (A), (B) and (C) provided by the family of higher Auslander algebras of type $\mathbb{A}$ offers the opportunity to use insights or techniques from one of these subjects to the benefit of another. In this work we give a first illustration of the possibilities that arise from the interaction between higher Auslander-Reiten theory (A) and wrapped Floer theory (C). Namely, we provide a symplectic proof of the derived Morita equivalence between the k-algebras $A_{n, d}$ and $A_{n, n-d}$ obtained by Beckert in [Bec18] by means of a rather involved calculus of derivators.

Theorem 2 (Theorems 3.3.3 and 3.4.3). Let $n \geq d \geq 1$. There is a commutative diagram of quasiequivalences of triangulated $A_{\infty}$-categories

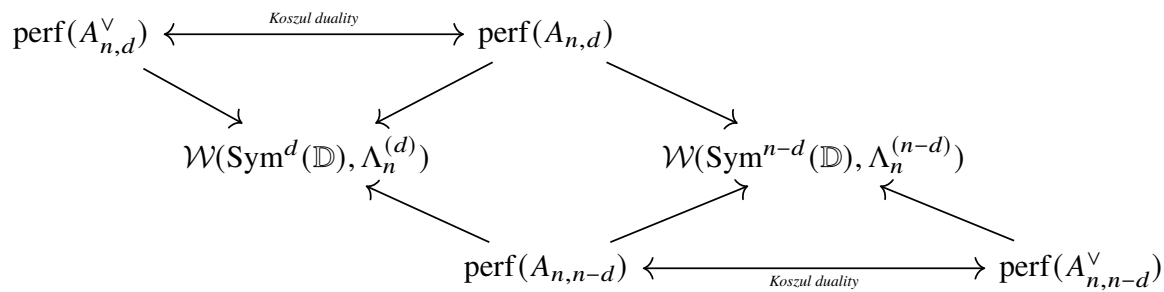

where, by convention, $\mathcal{W}\left(\operatorname{Sym}^{0}(\mathbb{D}), \Lambda_{n}^{(0)}\right)=\operatorname{perf}(\mathbf{k})$, and $A_{n, d}^{\vee}$ and $A_{n, n-d}^{\vee}$ denote the derived Koszul duals of the (augmented) $\mathbf{k}$-algebras $A_{n, d}$ and $A_{n, n-d}$, respectively.

The proofs of Theorem 1 and Theorem 2 exploit results of Auroux [Aur10a] which allow us calculate the cochain complexes of morphisms between objects in a distinguished subcategory of $\mathcal{W}\left(\operatorname{Sym}^{d}(\mathbb{D}), \Lambda_{n}^{(d)}\right)$ and to determine explicit classical generators of this partially wrapped Fukaya category. While the triangles of quasi-equivalences in Theorem 2 are direct consequences of derived Koszul duality for homologically smooth and proper algebras, the diamond of quasi-equivalences in the 
middle is due to an additional symmetry: first, note that the binomial symmetry

$$
\left(\begin{array}{l}
n \\
d
\end{array}\right)=\left(\begin{array}{c}
n \\
n-d
\end{array}\right)
$$

can be concretely realised by the bijection

$$
\left(\begin{array}{l}
\mathbf{n} \\
d
\end{array}\right) \longleftrightarrow\left(\begin{array}{c}
\mathbf{n} \\
n-d
\end{array}\right), \quad I \longmapsto \mathbf{n} \backslash I,
$$

obtained by associating to a subset $I \subseteq \mathbf{n}$ of cardinality $d$ its complement. This bijection further mediates a correspondence of objects

$$
\begin{gathered}
\mathcal{W}\left(\operatorname{Sym}^{d}(\mathbb{D}), \Lambda_{n}^{(d)}\right) \longleftarrow\left(\begin{array}{c}
\mathbf{n} \\
d
\end{array}\right) \stackrel{\cong}{\longleftrightarrow}\left(\begin{array}{c}
\mathbf{n} \\
n-d
\end{array}\right) \longleftrightarrow \mathcal{W}\left(\operatorname{Sym}^{n-d}(\mathbb{D}), \Lambda_{n}^{(n-d)}\right) \\
\prod_{i \in I} L_{0, i} \longleftrightarrow \quad J \longmapsto \prod_{j \in J} L_{j-1, j},
\end{gathered}
$$

where the Lagrangians $L_{i j} \subset \mathbb{D}$ are the ones depicted in Figures 1 and 2, respectively. The proof of Theorem 2 simply amounts to verifying that these collections of Lagrangians generate the corresponding partially wrapped Fukaya categories and that, for a suitable choice of grading structures on them, the derived endomorphism algebras of their direct sums are quasi-isomorphic.

Remark. Let $\overline{\mathbb{H}} \subset \mathbb{C}$ be the closed upper half-plane and $\Lambda_{\mathbb{Z}} \subset \partial \overline{\mathbb{H}}$ the set of integer numbers. The results in this article can be easily extended to prove that there is a quasi-equivalence of triangulated $A_{\infty}$-categories

$$
\mathrm{D}^{\mathrm{fd}}\left(A_{\infty, d}\right) \stackrel{\simeq}{\longrightarrow} \mathcal{W}\left(\operatorname{Sym}^{d}(\overline{\mathbb{H}}), \Lambda_{\mathbb{Z}}^{(d)}\right)
$$

where $A_{\infty, d}$ is the (infinite-dimensional, locally unital) k-algebra defined in complete analogy to the higher Auslander algebras of type $\mathbb{A}$, considering instead the poset

$$
\left\{\begin{array}{l}
\mathbb{Z} \\
d
\end{array}\right\}=\left\{I \in \mathbb{Z}^{d} \mid i_{1} \leq \cdots \leq i_{d}\right\}
$$

of $d$-element multisubsets of $\mathbb{Z}$. When working over a field, the k-algebra $A_{\infty, d}$ was introduced originally in [JK19] as a higher-dimensional analogue of the mesh category of type $\mathbb{Z} A_{\infty}$ which appears prominently in classical Auslander-Reiten theory. Similarly, the partially wrapped Fukaya category $\mathcal{W}\left(\operatorname{Sym}^{d}\left(S^{1} \times\right.\right.$ $\left.\mathbb{R}_{\geq 0}\right), \Lambda_{n}^{(d)}$ ) associated to the $d$-fold symmetric product of a marked half-infinite cylinder is related to the higher-dimensional analogues of the tubes introduced in that same paper.

\subsection{Future work}

It is known [Boc16, HKK17, LP18] that the partially wrapped Fukaya category of a punctured surface is equivalent to the perfect derived category of a homologically smooth (but not necessarily proper) graded gentle algebra associated to a polygonal decomposition of the surface dual to a ribbon graph (Lagrangian skeleton) onto which the surface retracts. Conversely, the perfect derived category of every homologically smooth graded gentle algebra arises this way (see also [LP20a, Section 3.3] for a discussion of the special case of proper gentle algebras concentrated in degree 0). Therefore, the Fukaya categories of punctured Riemann surfaces provide a geometric way of studying the combinatorial structure of derived categories of graded gentle algebras. For example, in [LP20a] new derived equivalences between gentle algebras were discovered using this perspective, which subsequently led to a classification of all proper gentle algebras concentrated in degree 0 up to derived equivalence [APS19, Opp19].

An important feature of partially wrapped Fukaya categories of Weinstein manifolds is that, as envisioned by Kontsevich [Kon09] and further explored in [GPS18], they are expected to satisfy cosheaflike descent conditions. Namely, there should be a constructible cosheaf of $A_{\infty}$-categories defined on 
every Lagrangian skeleton of the Weinstein manifold whose $A_{\infty}$-category of global sections is equivalent to the partially wrapped Fukaya category of the Weinstein manifold. In the case of surfaces, this amounts to the fact that derived categories of gentle algebras can be exhibited as a colimit of a suitable diagram formed by perfect derived categories of $A_{n}$-quivers, which are to be interpreted as the stalks of the corresponding (constructible) cosheaf defined on a ribbon graph of the surface [HKK17, DK18, Dyc17a].

In subsequent work we exploit the interplay between $(B)$ and $(C)$ to further advance this programme: the simplicial combinatorics intrinsically present in (B) provide a systematic approach to local-to-global phenomena for the partially wrapped Fukaya categories of symmetric powers of Riemann surfaces. As a result, we expect to compute these partially wrapped Fukaya categories by gluing together perfect derived categories of higher-dimensional Auslander algebras of type $\mathbb{A}$. The present article thus focuses on the local aspects that enter into this picture. For completeness, we mention that the case of punctured disks is investigated in detail in [LP20b] from the point of view of homological mirror symmetry.

\subsection{Conventions}

We fix an arbitrary commutative ring $\mathbf{k}$. Concerning $A_{\infty}$-categories, we follow the notational conventions in Seidel's book [Sei08]. Given objects $x$ and $y$ in an $A_{\infty}$-category $\mathcal{A}$, we denote the cochain complex of morphisms from $x$ to $y$ by $\operatorname{hom}_{\mathcal{A}}(x, y)$ and write $\operatorname{hom}(x, y)=\operatorname{hom}_{\mathcal{A}}(x, y)$ if the ambient $A_{\infty}$-category is clear from the context. We also write

$$
\operatorname{Hom}_{\mathcal{A}}^{*}(x, y)=H^{*}\left(\operatorname{hom}_{\mathcal{A}}(x, y)\right)
$$

and $\operatorname{Hom}_{\mathcal{A}}(x, y)=\operatorname{Hom}_{\mathcal{A}}^{0}(x, y)$ to denote the graded $\mathbf{k}$-module and the plain $\mathbf{k}$-module of morphisms from $x$ to $y$, respectively, in the graded k-category $H^{*}(\mathcal{A})$ and the ungraded k-category $H^{0}(\mathcal{A})$. For differential graded k-categories we follow the conventions in [Kel06]; in particular, the graded Leibniz rule takes the form

$$
\partial(\psi \circ \varphi)=\partial(\psi) \circ \varphi+(-1)^{|\psi|} \psi \circ \partial(\varphi)
$$

whenever $\psi$ is a homogeneous morphism (we compose morphisms from right to left). Throughout the article we write '(ungraded) k-algebra' to mean an ordinary k-algebra concentrated in degree 0 . We denote the grading shift in the category of graded $\mathbf{k}$-modules by $V \mapsto V(1)$ to distinguish it from the shift of complexes.

\section{Preliminaries}

In this preliminary section we recall general aspects of the theory of Fukaya categories which are relevant to our investigation of the partially wrapped Fukaya categories associated to the symmetric products of the 2-dimensional unit disk.

\subsection{Fukaya categories of symmetric products of Riemann surfaces}

We begin with a brief overview of the different variants of the Fukaya category associated to the symmetric products of an arbitrary Riemann surface.

\subsubsection{Fukaya categories and wrapped Fukaya categories}

Let $\Sigma$ be a compact Riemann surface and $d \geq 1$ an integer. The complex structure $j$ on $\Sigma$ induces a complex structure $\operatorname{Sym}^{d}(j)$ on the $d$-fold symmetric product

$$
\operatorname{Sym}^{d}(\Sigma):=\underbrace{\Sigma \times \cdots \times \Sigma}_{d \text { times }} / \Im_{d}
$$


of $\Sigma$. Take $z \in \Sigma$ and let $D$ be the image of the embedding

$$
\operatorname{Sym}^{d-1}(\Sigma) \longrightarrow \operatorname{Sym}^{d}(\Sigma), \quad \mathbf{x} \longmapsto z+\mathbf{x}
$$

Recall that there is an isomorphism $H^{2}\left(\operatorname{Sym}^{d}(\Sigma)\right) \cong \bigwedge^{2} H^{1}(\Sigma) \oplus \mathbb{Z}$, where the direct summand $\mathbb{Z}$ is represented by the Poincare dual of $D$. We are chiefly interested in open symplectic manifolds of the form

$$
\operatorname{Sym}^{d}(\Sigma) \backslash D=\operatorname{Sym}^{d}(\Sigma \backslash\{z\}) .
$$

The divisor $D$ is ample, thus the complex manifold $\operatorname{Sym}^{d}(\Sigma)$ admits a symplectic form $\omega=\frac{1}{2 \pi} i F_{A}$, where $A$ is a connection on the line bundle $\mathcal{O}(k D)$ for some sufficiently large $k$. The symplectic form $\omega$ tames the complex structure on $\operatorname{Sym}^{d}(\Sigma)$ and lies in the cohomology class Poincaré dual to $k D$. Moreover, the restriction of $\omega$ to $\operatorname{Sym}^{d}(\Sigma)$ gives an exact symplectic form which is convex at infinity.

Let $p: \Sigma^{\times d} \rightarrow \operatorname{Sym}^{d}(\Sigma)$ be the branched covering map, which is ramified along the big diagonal in $\operatorname{Sym}^{d}(\Sigma)$. Let $\omega_{\Sigma}$ be a choice of a symplectic structure on $\Sigma$. In [Per08] Perutz constructs an exact Kähler form on $\operatorname{Sym}^{d}(() \Sigma)$ of the preceding type but with the additional property that $\omega=p_{*}\left(\omega_{\Sigma}^{\times d}\right)$ outside a small neighbourhood of the big diagonal. This additional property allows us to consider exact Lagrangians in $\operatorname{Sym}^{d}(\Sigma)$ of the form $L_{1} \times L_{2} \times \cdots \times L_{d}$, where $\left\{L_{i}\right\}$ is a collection of pairwise disjoint exact Lagrangians on $\Sigma$ (with respect to the Liouville form $\theta_{\Sigma}$ ).

Remark 2.1.1. The identification $\operatorname{Sym}^{d}(\mathbb{C}) \cong \mathbb{C}^{d}$ implies that $\operatorname{Sym}^{d}(\mathbb{D})$ is a ball of (real) dimension $2 d$. Moreover, the symplectic structure on $\operatorname{Sym}^{d}(\mathbb{D})$ is equivalent to the standard symplectic structure on the ball, since both tame the standard complex structure. We prefer to use the symplectic structure constructed via the symmetric product construction, in order to provide a diagrammatic description of the Lagrangians.

For an exact symplectic manifold $M$ with convexity at infinity, such as $\operatorname{Sym}^{d}(\Sigma)$, a rigorous construction of a Fukaya category of compact exact Lagrangians $\mathcal{F}(M)$ appears in Seidel's book [Sei08]. By construction, $\mathcal{F}(M)$ is an idempotent complete triangulated $A_{\infty}$-category which is linear over $\mathbf{k}$. There is also a rigorous construction of a larger $A_{\infty}$-category-due to Abouzaid and Seidel [AS10]that allows for noncompact exact Lagrangians with controlled behaviour at infinity, namely the wrapped Fukaya category $\mathcal{W}(M)$ in which $\mathcal{F}(M)$ embeds as a full subcategory.

\subsubsection{Partially wrapped Fukaya categories}

A fruitful extension of the foregoing considerations consists in equipping the symplectic manifold $M$ with stops and considering the resulting partially wrapped Fukaya categories. We review the technical setup of this construction. Let $\Sigma$ be a surface with nonempty boundary equipped with an exact area form $\omega_{\Sigma}$ and $\Lambda \subset \partial \Sigma$ a nonempty finite subset. Consider the complex manifold $\operatorname{Sym}^{d}(\Sigma)$ equipped with a symplectic form as already outline. Note that $\operatorname{Sym}^{d}(\Sigma)$ is a symplectic manifold with corners which can be considered as a Liouville sector as in [GPS19]. We let

$$
\Lambda^{(d)}=\bigcup_{p \in \Lambda} p+\operatorname{Sym}^{d-1}(\Sigma)
$$

be a union of symplectic hypersurfaces in the contact boundary of $\operatorname{Sym}^{d}(\Sigma)$ which we call stops. To these data Auroux [Aur10b] associates a partially wrapped Fukaya category $\mathcal{W}\left(\operatorname{Sym}^{d}(\Sigma), \Lambda^{(d)}\right)$; the general theory of partially wrapped Fukaya categories has been further developed by Ganatra, Pardon and Shende [GPS18] and Sylvan [Syl19a]. Auroux's construction of partially wrapped Fukaya categories was inspired by the bordered Heegaard Floer theory of Lipshitz, Ozsváth and Thurston [LOT18], as well as by the more familiar special case of the Fukaya-Seidel category [Sei08] associated to a Lefschetz fibration on an exact symplectic manifold. 


\subsubsection{Stop-removal sequences}

The three flavours of Fukaya categories already discussed are related by a commutative diagram of functors

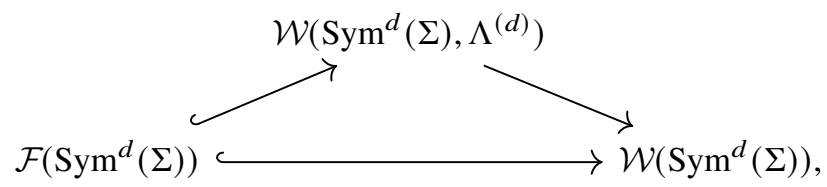

where the functor $\mathcal{F}\left(\operatorname{Sym}^{d}(\Sigma)\right) \hookrightarrow \mathcal{W}\left(\operatorname{Sym}^{d}(\Sigma), \Lambda^{(d)}\right)$ is fully faithful and so is its composite with the functor $\mathcal{W}\left(\operatorname{Sym}^{d}(\Sigma), \Lambda^{(d)}\right) \rightarrow \mathcal{W}\left(\operatorname{Sym}^{d}(\Sigma)\right)$. Moreover, the functor

$$
\mathcal{W}\left(\operatorname{Sym}^{d}(\Sigma), \Lambda^{(d)}\right) \longrightarrow \mathcal{W}\left(\operatorname{Sym}^{d}(\Sigma)\right)
$$

is the localisation at the full subcategory of $\mathcal{W}\left(\operatorname{Sym}^{d}(\Sigma), \Lambda^{(d)}\right)$ given by the union of the essential images of the so-called Orlov functors

$$
\iota_{p}: \mathcal{W}\left(\operatorname{Sym}^{d-1}(\Sigma), \Lambda_{p}^{(d-1)}\right) \longrightarrow \mathcal{W}\left(\operatorname{Sym}^{d}(\Sigma), \Lambda^{(d)}\right), \quad p \in \Lambda
$$

where $\Lambda_{p}=\Lambda \backslash\{p\}$; at the level of objects, the Orlov functor $\iota_{p}$ is obtained by taking the symmetric product with a sufficiently small arc around the point $p$. In this setting of symmetric products of Riemann surfaces, Auroux [Aur10b] proved that the image of the Orlov functors is generated by the objects supported near the stops (see also [Syl19a] for results in a much more general setting and [Syl19b] for further applications of these functors). For a fixed stop $p \in \Lambda$, the Orlov functor $\iota_{p}$ is part of a Drinfeld-Verdier localisation sequence

$$
\mathcal{W}\left(\operatorname{Sym}^{d-1}(\Sigma), \Lambda_{p}^{(d-1)}\right) \longleftrightarrow \mathcal{W}\left(\operatorname{Sym}^{d}(\Sigma), \Lambda^{(d)}\right) \longrightarrow \mathcal{W}\left(\operatorname{Sym}^{d}(\Sigma), \Lambda_{p}^{(d)}\right)
$$

which we refer to as the stop-removal sequence at $p$.

\subsubsection{Grading structures}

In general, the (cochain complexes of morphisms in the) partially wrapped Fukaya category $\mathcal{W}\left(\operatorname{Sym}^{d}(\Sigma), \Lambda^{(d)}\right)$ can only be $\mathbb{Z} / 2$-graded. If the first Chern class of $\operatorname{Sym}^{d}(\Sigma)$ satisfies $2 c_{1}=0$ (that is, if $\Sigma$ has genus 0 or if $d=1$ ), results in [Sei00] imply that $\mathcal{W}\left(\operatorname{Sym}^{d}(\Sigma), \Lambda^{(d)}\right)$ admits a $\mathbb{Z}$ grading; the possible $\mathbb{Z}$-gradings on $\mathcal{W}\left(\operatorname{Sym}^{d}(\Sigma), \Lambda^{(d)}\right)$ form a torsor over the first cohomology group $H^{1}\left(\operatorname{Sym}^{d}(\Sigma)\right) \cong H^{1}(\Sigma)$. Since in this article we only study the case when $\Sigma$ is a disk (which has vanishing first cohomology), the partially wrapped Fukaya categories we consider admit a unique $\mathbb{Z}$-grading.

\subsubsection{Generators of the partially wrapped Fukaya category}

The following result [Aur10a] describes sets of generators of the partially wrapped Fukaya category $\mathcal{W}\left(\operatorname{Sym}^{d}(\Sigma), \Lambda^{(d)}\right)$ in terms of $\operatorname{arcs}$ in $\Sigma \backslash \Lambda$ :

Theorem 2.1.2 (Auroux). Let $\Sigma$ be a compact Riemann surface with nonempty boundary and $\Lambda$ be a finite set of points on its boundary. Let $L_{1}, \ldots, L_{n}$ be a collection of disjoint properly embedded arcs in $\Sigma \backslash \Lambda$ with end points in the boundary of $\Sigma$. Assume that $\Sigma \backslash\left\{L_{1}, \ldots, L_{n}\right\}$ is a disjoint union of disks, each of which contains at most one point of $\Lambda$. Then the partially wrapped Fukaya category $\mathcal{W}\left(\operatorname{Sym}^{d}(\Sigma), \Lambda^{(d)}\right)$ is generated, as an idempotent complete triangulated $A_{\infty}$-category, by the $\left(\begin{array}{l}n \\ d\end{array}\right)$ Lagrangian submanifolds $L_{I}=\prod_{i \in I} L_{i}$, where I ranges over the d-element subsets of $\{1, \ldots, n\}$.

Let us review Auroux's proof of Theorem 2.1.2. Fix $2 g+1$ points $p_{0}, \ldots, p_{2 g} \in \mathbb{C}$ and consider the compact surface $\bar{\Sigma}$ given by a branched double cover of $\mathbb{C} P^{1}$ branched over the $p_{i} \mathrm{~s}$ and $\infty$. Let $\Sigma$ be 
the surface obtained from $\bar{\Sigma}$ by puncturing it at the preimage of $\infty$. The surface $\Sigma$ comes with a 2-fold branched covering $\pi: \Sigma \rightarrow \mathbb{C}$. Consider the map

$$
f: \operatorname{Sym}^{d}(\Sigma) \longrightarrow \mathbb{C}, \quad\left[z_{1}, \ldots, z_{d}\right] \longmapsto \pi\left(z_{1}\right)+\cdots+\pi\left(z_{d}\right)
$$

The map $f$ is a Lefschetz fibration whose critical points are tuples $\left[q_{s_{1}}, \ldots, q_{s_{d}}\right]$, where $s=\left\{s_{1}, \ldots, s_{d}\right\}$ is a $d$-element subset of $\left\{p_{0}, \ldots, p_{2 g}\right\}$ and $\pi\left(q_{s_{i}}\right)=p_{s_{i}}$. That is, the critical points consist of $d$ tuples of distinct critical points of $\pi$. By adding more stops if necessary, the generators specified in Theorem 2.1.2 can be identified with thimbles of the Lefschetz fibration $f$ and, moreover, the resulting partially wrapped Fukaya category is quasi-equivalent to the Fukaya-Seidel category of $f$. A celebrated result of Seidel [Sei08] shows that these thimbles generate the Fukaya-Seidel category of $f$. Removing the additional stops, if there are any, corresponds to a localisation of the Fukaya-Seidel category of $f$. Auroux leverages these considerations to prove that the product Lagrangians listed in Theorem 2.1.2 are indeed sufficient to generate the partially wrapped Fukaya category $\mathcal{W}\left(\operatorname{Sym}^{d}(\Sigma), \Lambda^{(d)}\right)$ as an idempotent complete triangulated $A_{\infty}$-category.

In the particular case of the pair $\left(\operatorname{Sym}^{d}(\mathbb{D}), \Lambda_{n}\right)$, Auroux's Lefschetz fibration can be described concretely as follows: first, consider the $(n+1)$-fold branched covering map

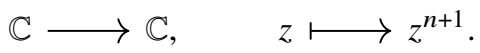

This is chosen so that the preimage of $1 \in \mathbb{C}$ is the set $\Lambda_{n}=\left\{p_{0}, p_{1}, \ldots, p_{n}\right\}$ of stops, which is contained in the boundary of the unit disk. Therefore, the Fukaya-Seidel category [Sei08] of a morsification of this map is quasi-equivalent to the partially wrapped Fukaya category $\mathcal{W}_{n}^{(1)}=\mathcal{W}\left(\mathbb{D}, \Lambda_{n}\right)$. A concrete morsification is given by

$$
f_{n, 1}: \mathbb{C} \longrightarrow \mathbb{C}, \quad z \longmapsto z^{n+1}-\varepsilon(n+1) z
$$

for some $\varepsilon>0$, so that the $n$ distinct critical points of $f_{n, 1}$ lie on the circle of radius $\sqrt[n]{\varepsilon}$. Consider now the Lefschetz fibration

$$
f_{n, d}: \operatorname{Sym}^{d}(\mathbb{C}) \rightarrow \mathbb{C}, \quad\left[z_{1}, \ldots, z_{d}\right] \mapsto \sum_{i=1}^{d} f_{n, 1}\left(z_{i}\right)=\sum_{i=1}^{d} z_{i}^{n+1}-\varepsilon(n+1) z_{i} .
$$

Essentially by construction (see [Aur10a, Sections 3.3.1 and 3.3.2]), the Fukaya-Seidel category of $f_{n, d}$ is quasi-equivalent to the partially wrapped Fukaya category $\mathcal{W}_{n}^{(d)}=\mathcal{W}\left(\operatorname{Sym}^{d}(\mathbb{D}), \Lambda_{n}^{(d)}\right)$. Under the identification

$$
\operatorname{Sym}^{d}(\mathbb{C}) \stackrel{\cong}{\longrightarrow} \mathbb{C}^{d}, \quad\left[z_{1}, \ldots, z_{d}\right] \longmapsto\left(e_{1}\left(z_{1}, \ldots, z_{d}\right), \ldots, e_{d}\left(z_{1}, \ldots, z_{d}\right)\right),
$$

where $e_{i}$ is the $i$ th elementary symmetric polynomial (the sum of all distinct products of $i$ variables), we can express the function $f_{n, d}$ (and its morsification) in terms of the coordinates of $\mathbb{C}^{d}$ using Newton's identities. For example, for $n=d=2$ we obtain the map

$$
\mathbb{C}^{2} \longrightarrow \mathbb{C}, \quad(u, v) \longmapsto u^{3}-3 u v-3 \varepsilon u .
$$

\subsubsection{Derived endomorphism algebras of generators}

Let $L_{1}, \ldots, L_{n}$ be arcs in $\Sigma \backslash \Lambda_{n}$ which satisfy the assumptions in Theorem 2.1.2, with the additional condition that each disk in the decomposition $\Sigma \backslash\left(\cup L_{i}\right)$ contains exactly one stop. Suppose for a moment that the ground ring $\mathbf{k}$ has characteristic 2, so that the results in [Aur10b] apply verbatim. In this context, Auroux provides a differential $\mathbb{Z} / 2$-graded model for the derived endomorphism algebra $\mathcal{A}=\bigoplus_{I, J} \operatorname{hom}\left(L_{I}, L_{J}\right)$ of the associated generator of the partially wrapped Fukaya category 
$\mathcal{W}\left(\operatorname{Sym}^{d}(\Sigma), \Lambda^{(d)}\right)$. By picking a particularly nice perturbation scheme adapted to this setup, he shows that there are no holomorphic $n$-gons for $n \geq 3$ with boundary on these generators, and therefore the higher products in the derived endomorphism algebra $\mathcal{A}$ vanish. Moreover, he determines all the holomorphic bigons and triangles that contribute to the product and the differential. In the end, Auroux establishes a quasi-isomorphism

$$
\mathcal{A}=\bigoplus_{I, J} \operatorname{hom}\left(L_{I}, L_{J}\right) \simeq \bigoplus_{I, J} \bigoplus_{\pi \in \subseteq_{d}} \operatorname{hom}\left(L_{I}, L_{J}\right)^{\pi},
$$

where

$$
\operatorname{hom}\left(L_{I}, L_{J}\right)^{\pi}=\operatorname{hom}\left(L_{i_{1}}, L_{j_{\pi(1)}}\right) \otimes \operatorname{hom}\left(L_{i_{2}}, L_{j_{\pi(2)}}\right) \otimes \cdots \otimes \operatorname{hom}\left(L_{i_{d}}, L_{j_{\pi(d)}}\right)
$$

and $\operatorname{hom}\left(L_{i}, L_{j}\right)$ is the free $\mathbf{k}$-module generated by all Reeb chords $L_{i} \rightarrow L_{j}$ induced by the Reeb flow along the various boundary components of $\Sigma \backslash \Lambda_{n}$. The differential $\mathbb{Z} / 2$-graded $\mathbf{k}$-algebra on the righthand side can be identified with the strands algebra arising in the context of the bordered Heegaard Floer homology of Lipshitz, Ozsváth and Thurston [LOT18]. We emphasise that in general the above direct sum decomposition of the strands algebra is preserved neither by the differential nor the multiplication operation, which is a source of difficulties.

In arbitrary characteristic, Auroux's description is still relevant. Indeed, this counting of holomorphic polygons does not depend on the ground ring nor on a possible choice of grading structure on $\operatorname{Sym}^{d}(\Sigma)$. However, there are signs involved in the differential and the composition of morphisms, which arise from the orientations of the various moduli spaces of holomorphic disks. In the case where $\Sigma=\mathbb{D}$, which is the only concern of this article, we approach this problem of determining signs by providing an explicit characteristic-free lift of the strands algebra from [LOT18] by means of a simple modification of a construction of Khovanov [Kho14] and showing that any other lift gives rise to an isomorphic algebra.

\subsection{The partially wrapped Fukaya categories $\mathcal{W}_{n}^{(d)}$}

Let $\mathbb{D}$ be the 2-dimensional unit disk and $\Lambda_{n}=\left\{p_{0}, p_{1}, \ldots, p_{n}\right\}$ be a set of stops on its boundary, where $n \geq 0$. While from the point of view of symplectic topology the precise position of the stops does not matter, for definiteness we often let $\Lambda_{n}$ be the set of $(n+1)$ th roots of unity. In this section we begin our analysis of the partially wrapped Fukaya categories

$$
\mathcal{W}_{n}^{(d)}:=\mathcal{W}\left(\operatorname{Sym}^{d}(\mathbb{D}), \Lambda_{n}^{(d)}\right), \quad d \geq 1 .
$$

Note that $\operatorname{Sym}^{d}(\mathbb{D})$ has Liouville completion $\operatorname{Sym}^{d}(\mathbb{C}) \cong \mathbb{C}^{n}$, which is an exact symplectic manifold with vanishing first Chern class. As explained in Section 2.1.4, the partially wrapped Fukaya category $\mathcal{W}_{n}^{(d)}$ admits a $\mathbb{Z}$-grading, and the vanishing of the first cohomology group $H^{1}\left(\operatorname{Sym}^{d}(\mathbb{D})\right) \cong H^{1}(\mathbb{D})$ implies that this $\mathbb{Z}$-grading is unique. We equip $\mathcal{W}_{n}^{(d)}$ with this canonical $\mathbb{Z}$-grading throughout the article. In $\operatorname{Sym}^{d}(\mathbb{D})$, we will consider Lagrangians $L$ of the form $L=\prod_{i=1}^{d} L_{i}$, where $L_{i}$ are pairwise disjoint arcs in $\mathbb{D} \backslash \Lambda_{n}$. These are all contractible, and thus the choice of a grading structure on such a Lagrangian is unique up to the natural action of $\mathbb{Z}$ which corresponds to the shift functor in $\mathcal{W}_{n}^{(d)}$. Finally, if $n<d$ it can be shown - by applying the stop-removal sequence (2.1.1) recursively and keeping in mind that the (fully) wrapped Fukaya category $\mathcal{W}^{(d)}=\mathcal{W}\left(\mathbb{C}^{n}\right)$ is trivial - that the partially wrapped Fukaya category $\mathcal{W}_{n}^{(d)}$ is trivial. Thus, our interest mostly lies in the case $n \geq d \geq 1$.

\subsection{The partially wrapped Fukaya category $\mathcal{W}_{n}^{(1)}$}

\subsubsection{Combinatorial coordinates}

We assume that the orientation of $\mathbb{D}$ is such that, if embedded in the plane, its boundary is oriented counterclockwise. For combinatorial reasons, we assume that the cyclic order on the labels of the stops 


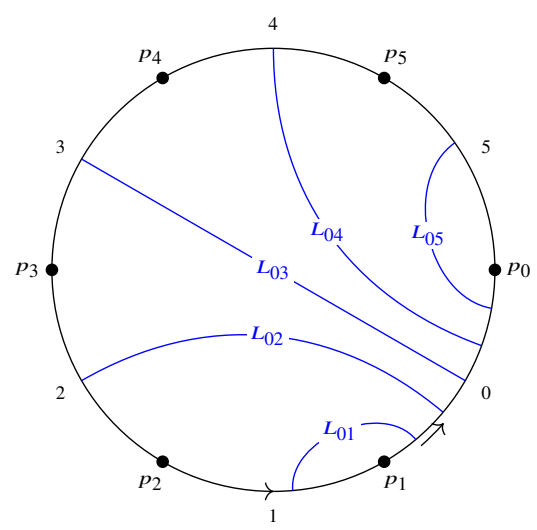

Figure 1. The generators $L_{01}, \ldots, L_{0 n}$ of $\mathcal{W}_{n}^{(1)}=\mathcal{W}\left(\mathbb{D}, \Lambda_{n}\right)$ in the case where $n=5$.

$p_{i} \in \Lambda_{n}$ induced by the orientation of the disk's boundary agrees with the opposite of the natural cyclic order on the set $\{0,1, \ldots, n\}$. We denote by $i$ the boundary component of $\mathbb{D} \backslash \Lambda_{n}$ which lies between the points $p_{i}$ and $p_{i+1}$. In particular, if $n=0$ then there is a single boundary component labelled 0 .

For $0 \leq i<j \leq n$, we let $L_{i j}$ be a properly embedded arc in $\mathbb{D} \backslash \Lambda_{n}$ whose end points lie on the components of $\partial \mathbb{D} \backslash \Lambda_{n}$ labelled $i$ and $j$. With some abuse of notation, we identify these Lagrangians with objects of the partially wrapped Fukaya category $\mathcal{W}_{n}^{(1)}$, keeping in mind that one also has to choose grading structures on them. Objects with the same underlying Lagrangians with different grading structures are related by the shift functor. The morphisms between disjoint Lagrangians are given by (composites of) Reeb chords corresponding to the Reeb flow along the boundary of the disk, which is the rotational flow in the counterclockwise direction. More generally, there are additional morphisms induced by intersection points.

The Lagrangians $L_{01}, \ldots, L_{0 n}$ play a particularly important role in this article. We choose isotopy classes of these Lagrangians so that they are mutually disjoint and partition $\mathbb{D}$ into a disjoint union of (topological) disks, each of which contains exactly one stop in $\Lambda_{n}$. By Theorem 2.1.2, these Lagrangians generate the partially wrapped Fukaya category $\mathcal{W}_{n}^{(1)}$ as an idempotent complete triangulated $A_{\infty}$ category (with respect to arbitrarily chosen grading structures on them). See Figure 1 for an example in the case where $n=5$.

\subsubsection{The quasi-equivalence $\mathcal{W}_{n}^{(1)} \simeq \operatorname{perf}\left(A_{n, 1}\right)$}

Let $n \geq 1$. Recall that $A_{n, 1}$ denotes the path $\mathbf{k}$-algebra of the quiver

$$
1 \rightarrow 2 \rightarrow \cdots \rightarrow n
$$

We view $A_{n, 1}$ as an $A_{\infty}$-algebra concentrated in degree 0 and vanishing higher products. We claim that there is an isomorphism of $A_{\infty}$-algebras

$$
\bigoplus_{i, j=1}^{n} \operatorname{hom}\left(L_{0 i}, L_{0 j}\right) \stackrel{\simeq}{\longrightarrow} A_{n, 1}
$$

and, consequently, a quasi-equivalence of triangulated $A_{\infty}$-categories

$$
\operatorname{perf}\left(A_{n, 1}\right) \stackrel{\simeq}{\longrightarrow} \mathcal{W}_{n}^{(1)}
$$

Indeed, as explained at the beginning of Section 2.3.1, since the Lagrangians $L_{01}, \ldots, L_{0 n}$ do not intersect in the interior of the disk, the only morphisms between them in $\mathcal{W}_{n}^{(1)}$ are given by Reeb chords 
corresponding to the Reeb flow along the boundary of the disk:

$$
L_{01} \rightarrow L_{02} \rightarrow \cdots \rightarrow L_{0 n}
$$

Note that there are no relations between these morphisms. Moreover, since this quiver is a tree, we can choose grading structures on the Lagrangians to ensure that all of the morphisms have degree 0 (for example, by choosing arbitrary grading structures and then shifting them as necessary). Therefore, with respect to the aforementioned choice of grading structures, the natural map

$$
\bigoplus_{i, j=1}^{n} \operatorname{hom}\left(L_{0 i}, L_{0 j}\right) \stackrel{\cong}{\longrightarrow} \bigoplus_{i, j}^{n} H^{0}\left(\operatorname{hom}\left(L_{0 i}, L_{0 j}\right)\right)
$$

is an isomorphism of $A_{\infty}$-algebras, and moreover, there is an isomorphism of (ungraded) k-algebras

$$
\bigoplus_{i, j}^{n} H^{0}\left(\operatorname{hom}\left(L_{0 i}, L_{0 j}\right)\right) \cong A_{n, 1}
$$

in particular, the $A_{\infty}$-algebra $\bigoplus_{i, j=1}^{n} \operatorname{hom}\left(L_{0 i}, L_{0 j}\right)$ is in fact an (ungraded) k-algebra. This proves the claim.

For later use we note that with respect to appropriately chosen grading structures on the Lagrangians $L_{0 i}, 1 \leq i \leq n$, this argument establishes the existence of isomorphisms of graded $\mathbf{k}$-modules

$$
\operatorname{hom}\left(L_{0 i}, L_{0 j}\right) \cong \begin{cases}\mathbf{k}(0) & i \leq j \\ 0 & \text { otherwise }\end{cases}
$$

In particular, the differential graded $\mathbf{k}$-algebra $\bigoplus_{i, j=1}^{n} \operatorname{hom}\left(L_{0, i}, L_{0, j}\right)$ is in fact an (ungraded) $\mathbf{k}$-algebra.

\subsubsection{The quasi-equivalence $\mathcal{W}_{n}^{(1)} \simeq \operatorname{perf}\left(A_{n, n-1}\right)$}

Following [HKK17, Section 3.3] and [LP20b, Section 2.1], consider the Lagrangians $L_{i-1, i}, 1 \leq i \leq n$, in $\mathbb{D} \backslash \Lambda_{n}$, embedded in such a way that they are mutually disjoint and partition $\mathbb{D}$ into a disjoint union of disks, each of which contains exactly one stop in $\Lambda_{n}$; for reference, the situation is depicted in Figure 2 in the case where $n=5$. By Theorem 2.1.2, these Lagrangians generate the partially wrapped Fukaya category $\mathcal{W}_{n}^{(1)}=\mathcal{W}\left(\mathbb{D}, \Lambda_{n}\right)$ as an idempotent complete triangulated $A_{\infty}$-category. Moreover, since the Lagrangians $L_{i-1, i}, 1 \leq i \leq n$, do not intersect in the interior of the disk, the only morphisms in $\mathcal{W}_{n}^{(1)}$

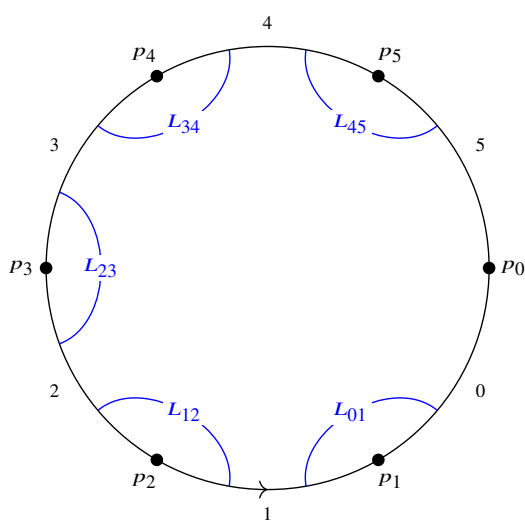

Figure 2. The generators $L_{01}, L_{12}, \ldots, L_{n-1, n}$ of $\mathcal{W}\left(\mathbb{D}, \Lambda_{n}\right)$ in the case where $n=5$. 
between them are given by Reeb chords corresponding to the Reeb flow along the boundary of the disk:

$$
L_{n-1, n} \rightarrow \cdots \rightarrow L_{12} \rightarrow L_{01}
$$

Note that the composite of any two consecutive of these morphisms vanishes, because the corresponding Reeb chords cannot be composed. Also, since the quiver is a tree, we can choose grading structures on the Lagrangians $L_{i-1, i}, 1 \leq i \leq n$, to ensure that all the morphisms between them have degree 0 . The resulting k-algebra is isomorphic to the higher Auslander algebra $A_{n-1, n}$. This establishes the existence of the required quasi-equivalences. For later use, we note that this argument shows that there is an isomorphism of graded $\mathbf{k}$-modules

$$
\operatorname{hom}\left(L_{j-1, j}, L_{i-1, i}\right) \cong \begin{cases}\mathbf{k}(0) & 0 \leq j-i \leq 1 \\ 0 & \text { otherwise }\end{cases}
$$

with respect to appropriate grading structures on the corresponding Lagrangians. In particular, the differential graded k-algebra $\bigoplus_{i, j=1}^{n} \operatorname{hom}\left(L_{j-1, j}, L_{i-1, i}\right)$ is in fact and (ungraded) k-algebra.

\section{Fukaya categories of symmetric products of disks}

We fix natural numbers $n \geq d \geq 1$. We recall from [OT12] an equivalent definition of Iyama's higher Auslander algebras of type $\mathbb{A}$ which is the most convenient for our present purposes. It is straightforward to verify that this definition agrees with that given in the introduction to this article.

Definition. The $d$-dimensional Auslander algebra of type $\mathbb{A}_{n-d+1}$ is the (ungraded) k-algebra with underlying k-module

$$
A_{n, d}:=\bigoplus_{I \leq J} \mathbf{k} \cdot f_{J I} /\left\langle f_{J I} \mid \exists a: j_{a} \geq i_{a+1}\right\rangle,
$$

where $I$ and $J$ range over the poset $\left(\begin{array}{l}\mathbf{n} \\ d\end{array}\right)$, equipped with the apparent multiplication law

$$
f_{K J^{\prime}} \cdot f_{J I}= \begin{cases}f_{K I} & \text { if } J=J^{\prime} \\ 0 & \text { otherwise }\end{cases}
$$

In particular, $A_{n, d}$ is a monomial quotient of the incidence k-algebra of the poset $\left(\begin{array}{c}\mathbf{n} \\ d\end{array}\right)$.

Remark. If $n=d$, then the corresponding higher Auslander algebra $A_{n, n}$ is isomorphic to the ground commutative ring $\mathbf{k}$, since the poset $\left(\begin{array}{c}\mathbf{n} \\ n\end{array}\right)$ consists of a single element.

This section is devoted to the proofs of our main results, which explicitly determine the derived endomorphism algebras

$$
\mathcal{A}_{n, d}:=\bigoplus_{I, J} \operatorname{hom}\left(L_{I}, L_{J}\right), \quad \mathcal{A}_{n, d}^{\vee}:=\bigoplus_{J, I} \operatorname{hom}\left(L_{J}^{\vee}, L_{I}^{\vee}\right)
$$

and

$$
\mathcal{A}_{n, d}^{\sharp}:=\bigoplus_{J, I} \operatorname{hom}\left(L_{J}^{\sharp}, L_{I}^{\sharp}\right)
$$

associated to three different sets of generators $\left\{L_{I}\right\},\left\{L_{I}^{\vee}\right\}$ and $\left\{L_{J}^{\sharp}\right\}$ of the partially wrapped Fukaya category $\mathcal{W}_{n}^{(d)}$. The collections $\left\{L_{I}^{\vee}\right\}$ and $\left\{L_{I}^{\sharp}\right\}$ agree up to shift:

$$
L_{I}^{\sharp}=L_{I}^{\vee}[-\mathrm{rk}(I)],
$$

where $\operatorname{rk}(I)$ is the (normalised) rank of $I \in\left(\begin{array}{l}\mathbf{n} \\ d\end{array}\right)$; in particular, their underlying Lagrangians in $\operatorname{Sym}^{d}(\mathbb{D} \backslash$ $\left.\Lambda_{n}^{(d)}\right)$ are equal. More precisely, we prove the following statements: 
1. In Theorem 3.2.3 we prove that there is a quasi-isomorphism of differential graded $\mathbf{k}$-algebras

$$
\mathcal{A}_{n, d} \stackrel{\simeq}{\longrightarrow} A_{n, d}
$$

which results in a quasi-equivalence of triangulated $A_{\infty}$-categories

$$
\operatorname{perf}\left(A_{n, d}\right) \stackrel{\simeq}{\longrightarrow} \mathcal{W}_{n}^{(d)}, \quad A_{n, d} \longmapsto \bigoplus L_{I}
$$

2. In Theorems 3.3.3 and 3.4.3, respectively, we prove that there are isomorphisms of differential graded k-algebras

$$
\mathcal{A}_{n, d}^{\vee} \cong A_{n, d}^{\vee} \text { and } \quad \mathcal{A}_{n, d}^{\sharp} \cong A_{n, n-d},
$$

where we remind the reader that the Koszul dual algebra $A_{n, d}^{\vee}$ is generated in cohomological degrees 0 and 1 . These result in quasi-equivalences of triangulated $A_{\infty}$-categories

$$
\operatorname{perf}\left(A_{n, d}^{\vee}\right) \stackrel{\simeq}{\longrightarrow} \mathcal{W}_{n}^{(d)}, \quad A_{n, d}^{\vee} \longmapsto \bigoplus L_{I}^{\vee}
$$

and

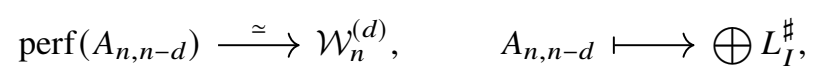

respectively. Moreover, the collections $\left\{L_{I}\right\}$ and $\left\{L_{I}^{\vee}\right\}$ are Koszul dual to one another, and hence the associated quasi-equivalences $\operatorname{perf}\left(A_{n, d}\right) \stackrel{\simeq}{\rightarrow} \mathcal{W}_{n}^{(d)}$ and $\operatorname{perf}\left(A_{n, d}^{\vee}\right) \stackrel{\simeq}{\rightarrow} \mathcal{W}_{n}^{(d)}$ are intertwined by Koszul duality.

\subsection{The strands algebra $\mathcal{B}_{n, d}$}

For the sake of completeness, we provide a lift of the 'strands algebra with $d$ strands $n$ places' introduced in [LOT18] which takes into account the fact that we work in arbitrary characteristic. Our construction can be interpreted as a decorated version of a construction of Khovanov [Kho14]. As we will explain, the strands algebra is a combinatorially defined differential graded $\mathbf{k}$-algebra which we eventually prove to be quasi-isomorphic to the derived endomorphism algebra $\mathcal{A}_{n, d}$ of an appropriate set $\left\{L_{I}\right\}$ of generators of the partially wrapped Fukaya category $\mathcal{W}_{n}^{(d)}$.

\subsubsection{The Bruhat order on $\mathfrak{S}_{d}$}

As originally observed in [LOT18], the strands algebra is closely related to the Bruhat order on the symmetric group $\mathfrak{S}_{d}$. We recall from [BB05] the relevant terminology. By a word we mean a (finite) word in the alphabet $\left\{s_{1}, \ldots, s_{d-1}\right\} \subset \mathfrak{S}_{d}$ given by the simple transpositions $s_{a}=(a+1, a)$ (note that we read words from right to left, since we want to relate these to the composition of morphisms in the Fukaya category). A word $w^{\prime}$ is a subword of a word $w$ if $w^{\prime}$ is obtained from $w$ by deleting some of its letters; in particular, the empty word is a subword of every word. A reduced expression for a permutation $\pi \in \mathfrak{S}_{d}$ is factorisation of $\pi$ as a word of minimal length; a word is reduced if it is a reduced expression for some permutation. The length of a permutation is the length of any of its reduced expressions. Equivalently, the length of $\pi \in \mathfrak{S}_{d}$ is the number

$$
\operatorname{inv}(\pi)=\#\{1 \leq a<b \leq d \mid \pi(b)<\pi(a)\}
$$

of inversions in $\pi$ (see [BB05]). The relation $\pi^{\prime} \leq \pi$ holds in the Bruhat order on $\mathfrak{S}_{d}$ if and only if there exists a reduced expression of $\pi$ which contains a reduced expression of $\pi^{\prime}$ as a subword (see [BB05]). Equivalently, $\pi^{\prime} \leq \pi$ if and only if every reduced expression of $\pi$ contains a reduced expression of $\pi^{\prime}$ as 

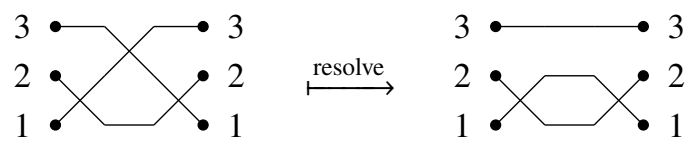

Figure 3. Example of a resolution which results in a strand diagram in which two strands cross more than once. On the left, the reduced word (21)(32)(21); on the right, the nonreduced word (21)e(21).

a subword (see [BB05, Corollary 2.2.3]. The function $\pi \mapsto \operatorname{inv}(\pi)$ endows the Bruhat order on $\mathfrak{S}_{d}$ with the structure of a graded poset (see [BB05, Theorem 2.2.6]); the elements of degree 1 are precisely the simple transpositions. As is customary, we write $\pi^{\prime} \triangleleft \pi$ to denote a covering relation in the Bruhat order on $\mathfrak{\Im}_{d}$; equivalently, we write $\pi^{\prime} \triangleleft \pi$ if $\pi^{\prime}<\pi$ and $\operatorname{inv}(\pi)=\operatorname{inv}\left(\pi^{\prime}\right)+1$.

The Bruhat order on $\mathfrak{S}_{d}$ can be visualised diagrammatically as follows: a simple transposition can be depicted as a planar strand diagram; for example, the simple transpositions (21) and (32) in $\mathfrak{\Xi}_{d}$ correspond respectively to the diagrams

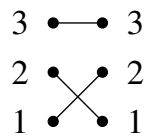

and

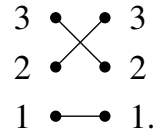

Each reduced word can be visualised as a strand diagram (obtained by concatenating the strands associated to simple transpositions) in which the crossings that appear are linearly ordered; the fact that the word is reduced corresponds to the fact that no two strands cross more than once. Deleting a letter from a word corresponds to resolving a crossing; note, however, that resolving a crossing may result in a strand diagram in which two strands cross more than once - see Figure 3 for an example. In these terms, the covering relation $\pi^{\prime} \triangleleft \pi$ holds in the Bruhat order on $\Im_{d}$ if and only if in any stranddiagram representation of $\pi$ in which no two strands cross more than once, we may resolve a single crossing (which is in fact uniquely determined; see [BB05, Corollary 1.4.4]) to obtain a strand-diagram representation of $\pi$ with the same property.

The Bruhat order on $\mathfrak{S}_{d}$ is an interval: the minimum element is the trivial permutation $e \in \mathfrak{S}_{d}$ and the maximum element is the permutation $a \mapsto d+1-a$. We see from [BB05, Proposition 2.3.4] shows that multiplication by the longest element induces an antiautomorphism of the Bruhat order on $\mathfrak{S}_{d}$; in particular, the duality principle applies in this context. More generally, a Bruhat interval is an interval

$$
\left[\pi^{\prime}, \pi\right]=\left\{\pi^{\prime \prime} \in \mathcal{S}_{d} \mid \pi^{\prime} \leq \pi^{\prime \prime} \leq \pi\right\}
$$

in the Bruhat order on $\mathfrak{S}_{d}$. It is a remarkable fact that provided that $\operatorname{inv}(\pi)-\operatorname{inv}\left(\pi^{\prime}\right) \geq 2$, there exists a regular cell complex, uniquely determined up to cellular homeomorphism, whose poset of cells is isomorphic to $\left[\pi^{\prime}, \pi\right]$ (by convention, $\pi^{\prime}$ corresponds to an empty cell); moreover, this regular cell complex is homeomorphic to a ball of dimension $\operatorname{inv}(\pi)-1$ (see [BB05, Theorem 2.7.12] for details).

Let $\pi \in \mathfrak{S}_{d}$ be a nontrivial permutation; following [BB05, Section 2.7], we associate a cochain complex to the Bruhat interval $[e, \pi]$ as follows. A balanced signature on the Hasse diagram of the Bruhat interval $[e, \pi]$ is an assignment $\pi^{\prime} \triangleleft \pi \mapsto \varepsilon\left(\pi^{\prime} \triangleleft \pi\right)$ of signs \pm 1 to the edges of the Hasse diagram of $[e, \pi]$ such that for every square

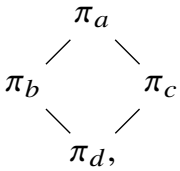

the equality

$$
\varepsilon\left(\pi_{b} \triangleleft \pi_{a}\right) \varepsilon\left(\pi_{d} \triangleleft \pi_{b}\right)+\varepsilon\left(\pi_{c} \triangleleft \pi_{a}\right) \varepsilon\left(\pi_{d} \triangleleft \pi_{c}\right)=0
$$

is satisfied (every Bruhat interval of length 2 is of this form; see in [BB05, Lemma 2.7.3]). 
Given a balanced signature $\varepsilon$ on the Hasse quiver of a Bruhat interval $[e, \pi]$, we define the free graded k-module $C[e, \pi]=\mathbf{k} \cdot[e, \pi]$, where $\pi \in[e, \pi]$ lies in degree $-\operatorname{inv}(\pi)$, and endow it with the differential

$$
\partial_{\varepsilon}\left(\pi^{\prime}\right)=\sum_{\pi^{\prime \prime} \triangleleft \pi^{\prime}} \varepsilon\left(\pi^{\prime \prime} \triangleleft \pi^{\prime}\right) \cdot \pi^{\prime \prime}
$$

for $\pi^{\prime} \in[e, \pi]$. It is straightforward to verify that $\partial_{\varepsilon}^{2}=0$. In terms of strand diagrams, this differential is given by the signed sum of all the permutations obtained from $\pi^{\prime}$ by resolving a single crossing in any of its strand-diagram representations, excluding resolutions which result in a diagram in which two strands cross more than once. Note that we can obtain a new balanced signature $\varepsilon^{\prime}$ from $\varepsilon$ by choosing an arbitrary permutation $\pi^{\prime} \in[e, \pi]$ and multiplying by -1 the signs of all the edges incident to $\pi^{\prime}$ in the Hasse quiver of $[e, \pi]$. In terms of the associated cochain complexes, this corresponds to the isomorphism

$$
\left(C[e, \pi], \partial_{\varepsilon}\right) \stackrel{\cong}{\longrightarrow}\left(C[e, \pi], \partial_{\varepsilon^{\prime}}\right), \quad \pi^{\prime} \longmapsto-\pi^{\prime},
$$

which fixes all the other generators of $C[e, \pi]$. In particular, this operation does not change the isomorphism type of the associated chain complex.

Suppose that $\operatorname{inv}(\pi) \geq 2$ and let $X=X[e, \pi]$ be the regular cell complex whose poset of cells is isomorphic to the Bruhat interval $[e, \pi]$. An arbitrary choice of orientation of the cells of $X$ allows us to identify $C[e, \pi]$ with the cellular chain complex $C(X ; \mathbf{k})$. In particular, the cochain complex $C[e, \pi]$ (which computes the reduced cellular homology of the topological ball $X$ ) is acyclic. The signs involved in the differential on $C[e, \pi]$ which arise via this procedure yield a balanced signature on the Hasse diagram of the Bruhat interval $[e, \pi]$. This is essentially the given proof of [BB05], where general Bruhat intervals are considered. We need the following slight refinement of this result:

Proposition 3.1.1. Let $\pi \in \mathfrak{S}_{d}$ be a nontrivial permutation. The following statements hold:

1. There exists a balanced signature $\varepsilon$ on the Hasse quiver of the Bruhat interval $[e, \pi]$ such that the corresponding cochain complex $\left(C[e, \pi], \partial_{\varepsilon}\right)$ is acyclic.

2. Let $\varepsilon$ and $\varepsilon^{\prime}$ be balanced signatures on the Hasse quiver of the Bruhat interval $[e, \pi]$. Then the corresponding chain complexes $\left(C[e, \pi], \partial_{\varepsilon}\right)$ and $\left(C[e, \pi], \partial_{\varepsilon^{\prime}}\right)$ are isomorphic.

In particular, for any choice of balanced signature $\varepsilon$ on the Hasse quiver of the Bruhat interval [e, $\pi]$, the cochain complex $\left(C[e, \pi], \partial_{\varepsilon}\right)$ is acyclic.

Proof. Statement 1 is an immediate consequence of [BB05, Corollary 2.7.14], which establishes the result in the case of integer coefficients (the general case follows by extending the coefficients to $\mathbf{k}$ ).

To prove Statement 2, we argue inductively. Let $\varepsilon$ and $\varepsilon^{\prime}$ be two different balanced signatures on the Hasse diagram of the Bruhat interval $[e, \pi]$. Recall that multiplying by -1 the signs associated to all the edges in the Hasse diagram which are incident to a given permutation does not change the isomorphism type of the corresponding cochain complex. We consider first the following special case: suppose that $\varepsilon$ and $\varepsilon^{\prime}$ agree on the half-open interval $[e, \pi)$. We claim that $\varepsilon^{\prime}$ is obtained from $\varepsilon$ by multiplying by -1 all the signs associated to all the edges incident to $\pi$ in $[e, \pi]$. If the Bruhat interval $[e, \pi]$ has length 1 - that is, if $\pi$ is a simple transposition - the claim is obvious. Suppose then that $\pi$ has length at least 2. The dual of [BB05, Corollary 2.2.8] implies that every pair of distinct edges $\pi_{a} \triangleleft \pi$ and $\pi_{b} \triangleleft \pi$ is part of some square

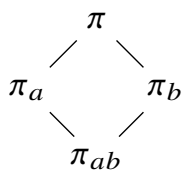

in the poset $[e, \pi]$. Clearly, once the signs at the edges $\pi_{a b} \triangleleft \pi_{a}$ and $\pi_{a b} \triangleleft \pi_{b}$ are fixed, there are exactly two balanced signatures on the Hasse diagram, and they are related to each other by multiplying by -1 the signs at the edges $\pi_{a} \triangleleft \pi$ and $\pi_{b} \triangleleft \pi$. Thus, it is enough to show that the value of a balanced signature on any such square determines the value on all other squares. If two squares share an edge incident to $\pi$, 
then it is clear that the restriction of the signature to either square determines the value on both squares, keeping in mind that the value at the bottom edges is already fixed. If this is not the case, then the two squares are arranged as follows (depicted by solid edges):

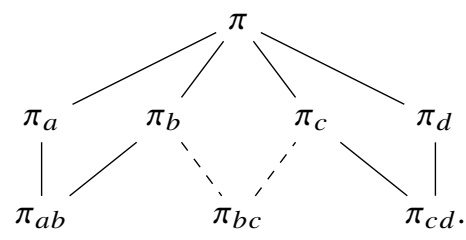

By the observation regarding pairs of edges incident to $\pi$, there are (at least) further covering relations in $[e, \pi]$, as indicated by the dashed edges. It is now clear that a balanced signature on the Hasse diagram in question is completely determined by its values on the edges which are not incident to $\pi$, together with its values at any square with maximum element $\pi$.

We now return to the general case. Inductively, set $1<k \leq \operatorname{inv}(\pi)$ and suppose that $\varepsilon$ and $\varepsilon^{\prime}$ are balanced signatures which agree on all edges in the Hasse diagram in $[e, \pi]$ between permutations of length at most $k-1$. Let $\pi^{\prime} \in[e, \pi]$ be a permutation of length $k$ and consider the Bruhat interval $\left[e, \pi^{\prime}\right]$. The foregoing argument shows that we can replace $\varepsilon^{\prime}$ by a new balanced signature which agrees with $\varepsilon$ on $\left[e, \pi^{\prime}\right]$ without changing the isomorphism type of the associated cochain complex. We can then repeat the argument for all remaining permutations $\pi^{\prime} \leq \pi$ of length $k$. By induction, the claim follows.

Remark 3.1.2. Let $\pi \in \mathfrak{S}_{d}$ be a nontrivial permutation. Combined with the proof of [BB05, Corollary 2.7.4], the proof of Proposition 3.1.1 shows that all possible balanced signatures on the Hasse diagram of the Bruhat order $\mathfrak{S}_{d}$ are obtained by choosing arbitrary orientations for the cells of the regular cell complex with incidence poset $[e, \pi]$.

Example 3.1.3. The Bruhat order on $\mathfrak{S}_{3}$ is isomorphic to the poset of cells associated to the following cell decomposition of the disk:

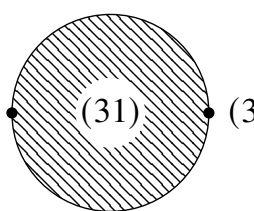

(231).

With respect to the choice of balanced signature in Figure 4, the cochain complex $C[e,(31)]$ is given by

$$
\mathbf{k} \cdot(31) \stackrel{\left(\begin{array}{c}
1 \\
-1
\end{array}\right)}{\longrightarrow} \mathbf{k} \cdot(321) \oplus \mathbf{k} \cdot(231) \stackrel{\left(\begin{array}{cc}
1 & 1 \\
-1 & -1
\end{array}\right)}{\longrightarrow} \mathbf{k} \cdot(21) \oplus \mathbf{k} \cdot(32) \stackrel{(11)}{\longrightarrow} \mathbf{k} \cdot e .
$$

The reader can easily verify that this cochain complex is acyclic.

\subsubsection{The definition of the strands algebra $\mathcal{B}_{n, d}$}

In [Kho14], Khovanov introduced a characteristic-free simplified variant of the strands algebra of [LOT18] which turns out to be contractible (in his definition, the unit is a coboundary). We introduce a decorated version of Khovanov's construction which provides a cohomologically nontrivial lift of the strands algebra of [LOT18] to arbitrary characteristic. We begin with a technical lemma:

Lemma 3.1.4. Let $I, J \in\left(\begin{array}{l}\mathbf{n} \\ d\end{array}\right)$. If nonempty, the subset

$$
\left\{\pi \in \mathfrak{\Im}_{d} \mid \forall 1 \leq a \leq d: i_{a} \leq j_{\pi(a)}\right\}
$$




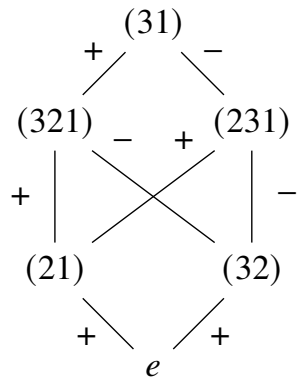

Figure 4. The Hasse diagram of Bruhat order on $\mathfrak{S}_{3}$, together with a choice of balanced signature.

is a Bruhat interval of the form $\left[e, \pi_{0}^{J I}\right]$, where $e \in \mathcal{S}_{d}$ is the trivial permutation. In particular, this subset is nonempty if and only if $I \leq J$.

Proof. Let $X=X_{J I}=\left\{\pi \in \mathfrak{S}_{d} \mid \forall a: i_{a} \leq j_{\pi(a)}\right\}$. Suppose that $X$ is nonempty. We show first that $X$ is downwards-closed. Since the symmetric group is finite, it suffices to show that if $\pi^{\prime} \triangleleft \pi$ is a covering relation in the Bruhat order on $\mathfrak{S}_{d}$ and $\pi \in X$, then $\pi^{\prime} \in X$. By [BB05, Lemma 2.1.4], we can write $\pi(b, a)=\pi^{\prime}$ for some $a<b$ such that $\pi(b)<\pi(a)$ and there does not exist $a<c<b$ such that $\pi(b)<\pi(c)<\pi(a)$. In particular,

$$
i_{a}<i_{b} \leq j_{\pi(b)}=j_{\pi^{\prime}(a)}<j_{\pi(a)}=j_{\pi^{\prime}(b)} .
$$

Therefore $\pi^{\prime} \in X$, as required. Finally, $X$ contains a maximum $\pi_{0}^{J I}$, constructed inductively as follows: if $d=1$, then $I=\{i\}$ and $J=\{j\}$ for some $i \leq j$, and $\pi_{0}^{J I}=e$ is the trivial permutation. For $d>1$, we let $\pi_{0}^{J I}(d)=\min \left\{a \mid i_{d} \leq j_{a}\right\}$ and define $I^{\prime}=I \backslash\left\{i_{d}\right\}$ and $J^{\prime}=J \backslash\left\{j_{\pi_{0}^{J I}(d)}\right\}$. Note that $I^{\prime} \leq J^{\prime}$. Inductively, we define $\pi_{0}^{J I}$ on $\{1, \ldots, d-1\}$ to be the unique maximal element of the poset

$$
\left\{\pi \in \mathfrak{S}_{d-1} \mid \forall 1 \leq a<d: i_{a} \leq j_{\pi(a)}\right\},
$$

which is nonempty since it contains the trivial permutation.

Notation 3.1.5. Let $I, J \in\left(\begin{array}{c}\mathbf{n} \\ d\end{array}\right)$. With some abuse of notation, we let $\left[e, \pi_{0}^{J I}\right]:=\emptyset$ whenever $I \not J J$.

Before giving the definition of the strands algebra we introduce a convenient diagrammatic language for visualising its generators.

Definition 3.1.6. Let $I, J \in\left(\begin{array}{l}\mathbf{n} \\ d\end{array}\right)$ be such that $I \leq J$. We view $I$ and $J$ as subsets of the real interval $[0, n+1]$. A strand diagram from $I$ to $J$ consists of a collection $\varphi: I \rightarrow J$ of $d$ properly embedded arcs in the rectangle $[0,1] \times[0, n+1]$, called strands, subject to the following restrictions:

1. Each strand is the graph of a monotonically increasing function

$$
[0,1] \longrightarrow[0, n+1]
$$

whose value at time 0 lies in $I$ and whose value at time 1 lies in $J$.

2. No two strands intersect at their endpoints.

3. No two strands intersect more than once. Moreover, all intersections are transversals.

4. At most two strands cross at any given time $t \in[0,1]$.

We write $\varphi(i)=j$ to indicate that $\varphi$ contains a strand connecting $i \in\{0\} \times I$ with $j \in\{1\} \times J$.

Remark 3.1.7. Let $I, J \in\left(\begin{array}{l}\mathbf{n} \\ d\end{array}\right)$ be such that $I \leq J$. More informally, a strand diagram from $I$ to $J$ consists of $d$ distinct curves in the rectangle $[0,1] \times[0, n+1]$ connecting exactly one point of $\{0\} \times I$ with exactly 
one point of $\{1\} \times J$. Moreover, strands are required to move strictly to the right and weakly upwards as time flows from 0 to 1 . Finally, we require that the number of crossings between any two strands be minimal, and that the crossings be positioned in such a way that the projection onto the time coordinate induces a linear order on the set of crossings in the strand diagram. When working in characteristic 2 , the last condition on the linear order of the crossings does not play any role in the definition of the strands algebra; we can then drop it and recover the strand diagrams from [LOT18].

Notation 3.1.8. Let $I, J \in\left(\begin{array}{l}\mathbf{n} \\ d\end{array}\right)$ be such that $I \leq J$. A strand diagram $\varphi: I \rightarrow J$ defines a permutation $\pi_{\varphi} \in\left[e, \pi_{0}^{J I}\right]$ given by $a \mapsto b$ if $\varphi\left(i_{a}\right)=j_{b}$; the linear order on the crossings of $\varphi$ (which correspond to inversions in $\pi_{\varphi}$ ) encodes the datum of a reduced expression of $\pi_{\varphi}$. In particular, $\operatorname{inv}\left(\pi_{\varphi}\right)$ is precisely the number of crossings between strands in $\varphi$. Conversely, for every reduced expression of a permutation $\pi \in\left[e, \pi_{0}^{J I}\right]$ there exists a (nonunique) strand diagram $\varphi=\varphi_{\pi}$ from $I$ to $J$ such that $\pi_{\varphi}=\pi$ and which encodes the given reduced expression of $\pi$.

Notation 3.1.9. Let $I, J \in\left(\begin{array}{c}\mathbf{n} \\ d\end{array}\right)$ and $\varphi: I \rightarrow J$ be a strand $\operatorname{diagram}$. We write $\operatorname{inv}(\varphi)=\operatorname{inv}\left(\pi_{\varphi}\right)$ for the number of crossings in $\varphi$.

Example 3.1.10. Let $n=5$ and $d=3$. Let $I=\{1,2,3\}$ and $J=\{3,4,5\}$. Consider the strand diagrams
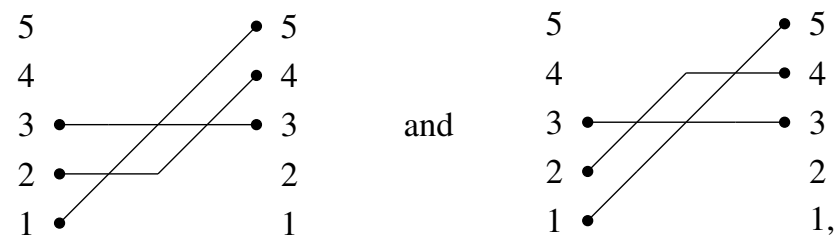

whose associated permutation is (31). From left to right, these strand diagrams encode the reduced expressions $(21)(32)(21)$ and (32)(21)(32), respectively.

Definition 3.1.11. Let $I, J \in\left(\begin{array}{l}\mathbf{n} \\ d\end{array}\right)$ such that $I \leq J$. Two strand diagrams $\varphi, \psi: I \rightarrow J$ are equivalent if $\pi_{\varphi}=\pi_{\psi}$ and the reduced expressions of this permutation induced by $\varphi$ and $\psi$ are equal.

Remark 3.1.12. The equivalence relation between strand diagrams can be phrased in terms of a natural notion of isotopy. Namely, two strand diagrams are equivalent if they are isotopic via an isotopy through strand diagrams in which the linear order induced on the crossings by their time coordinates remains unchanged.

Remark 3.1.13. Let $I, J \in\left(\begin{array}{c}\mathbf{n} \\ d\end{array}\right)$. By construction, equivalence classes of strand diagrams $I \rightarrow J$ are in bijection with reduced words in $\left[e, \pi_{0}^{J I}\right]$.

Notation 3.1.14. Let $I, J \in\left(\begin{array}{l}\mathbf{n} \\ d\end{array}\right)$ be such that $I \leq J$. We denote the equivalence class of strand diagrams $\varphi: I \rightarrow J$ such that $\pi_{\varphi}=e$ by $e_{J I}$.

We define a differential graded k-category with set of objects $\left(\begin{array}{l}\mathbf{n} \\ d\end{array}\right)$ as follows:

○ For $I, J \in\left(\begin{array}{l}\mathbf{n} \\ d\end{array}\right)$, we let $\operatorname{hom}(I, J)$ be the $\mathbf{k}$-module generated by equivalence classes of strand diagrams from $I$ to $J$, subject to the following relation: if $\varphi, \psi: I \rightarrow J$ are strand diagrams such that the linear orders induced on the respective crossings differ by a single transposition, then $\psi=-\varphi$.

$\circ$ Let $\varphi: I \rightarrow J$ and $\psi: J \rightarrow K$ be strand diagrams. We define

$$
\psi \circ \varphi= \begin{cases}\psi \varphi & \text { if } \operatorname{inv}\left(\pi_{\psi} \pi_{\varphi}\right)=\operatorname{inv}\left(\pi_{\psi}\right)+\operatorname{inv}\left(\pi_{\varphi}\right) \\ 0 & \text { otherwise }\end{cases}
$$

where the strand diagram $\psi \varphi$ is obtained by horizontal concatenation with the implicit reparametrisation of the time coordinate (recall that strands flow from left to right). More informally, the natural 
composition law has the following important caveat: if after concatenation of two strand diagrams the resulting strand diagram contains two strands which cross more than once, their composite vanishes.

- We endow hom $(I, J)$ with a grading by declaring the degree $|\varphi|$ of a strand diagram $\varphi$ to be $-\operatorname{inv}(\varphi)=-\operatorname{inv}\left(\pi_{\varphi}\right)-$ that is, minus its number of crossings. Since the composition operation is by design compatible with the grading, this yields a graded $\mathbf{k}$-category.

○ Finally, we endow the graded k-module hom $(I, J)$ with the differential

$$
\partial(\varphi)=\sum_{\psi \triangleleft \varphi} \varepsilon(\psi \triangleleft \varphi) \psi,
$$

where the sum ranges over all strand diagrams $\psi$ obtained from $\varphi$ by resolving a single crossing, excluding resolutions which result in a strand diagram in which two strands cross more than once, and $\varepsilon(\psi \triangleleft \varphi)$ is given by -1 to the number of crossings in $\varphi$ which happen after the crossing being resolved.

Definition-Proposition 3.1.15. The strands algebra with $d$ strands and $n$ places is the differential graded k-algebra

$$
\mathcal{B}_{n, d}:=\bigoplus_{I, J} \operatorname{hom}(I, J)
$$

Proof. It is straightforward to verify that the differential on $\operatorname{hom}(I, J)$ squares to 0 . To prove that the differential satisfies the graded Leibniz rule, we can proceed exactly as in the proof of [LOT18, Lemma 3.1], with a small modification. We include the argument for the sake of completeness. We define a larger graded k-algebra $\widetilde{\mathcal{B}}_{n, d}$ where we allow all generalised strand diagrams obtained by concatenating strand diagrams in which no two strands cross more than once. The degree of a generalised strand diagram is defined to be minus the number of crossings in the diagram (which may be larger than the number of inversions in the corresponding permutation). The product is given by horizontal concatenation of generalised strand diagrams (with no caveats) and the differential is defined exactly as for the strands algebra $\mathcal{B}_{n, d}$. Clearly, as a graded $\mathbf{k}$-algebra, $\mathcal{B}_{n, d}$ is a quotient of $\widetilde{\mathcal{B}}_{n, d}$. We verify that the differential on $\widetilde{\mathcal{B}}_{n, d}$ satisfies the graded Leibniz rule. Let $\varphi: I \rightarrow J$ and $\psi: J \rightarrow K$ be generalised strand diagrams. We analyse the expression

$$
\partial(\psi) \varphi+(-1)^{|\psi|} \psi \partial(\varphi)
$$

The term $\partial(\psi) \varphi$ corresponds precisely to the summand of $\partial(\psi \varphi)$ obtained by resolving crossings in $\psi \varphi$ which are crossings in $\psi$. Similarly, the remaining term $(-1)^{|\psi|} \psi \partial(\varphi)$ corresponds precisely to the summand of $\partial(\psi \varphi)$ obtained by resolving crossings in $\psi \varphi$ which are crossings in $\psi$; the sign $(-1)^{|\psi|}$ appears because $\partial(\varphi)$ does not take into account the number $-|\psi|$ of crossings in $\psi$, and all of these crossings happen after every crossing in $\varphi$. This shows that $\widetilde{\mathcal{B}}_{n, d}$ is a differential graded $\mathbf{k}$-algebra. To finish the proof, it remains to show that the graded submodule of $\widetilde{\mathcal{B}}_{n, d}$ generated by the generalised strand diagrams with at least one double crossing is in fact a differential graded ideal. It is clear that this graded k-submodule is a graded ideal. Also, if a strand diagram $\varphi$ has at least two double crossings, then $\partial(\varphi)$ has at least one double crossing and therefore lies in the ideal. Finally, if $\varphi$ has a single double crossing, then there are exactly two strand diagrams $\psi$ and $\psi^{\prime}$ which appear as summands of $\partial(\varphi)$ that do not lie in the ideal; we claim that these terms cancel each other. Indeed, since the permutations $\pi_{\psi}$ and $\pi_{\psi^{\prime}}$ clearly agree, $\psi$ and $\psi^{\prime}$ differ from each other by a sign as generators of $\widetilde{\mathcal{B}}_{n, d}$; this sign is given by -1 to the number of crossings that appear strictly between the two crossings which are part of the unique double crossing in $\varphi$. The claim follows.

Example 3.1.16. Let $n=5$ and $d=4$. Let $I=\{1,2,3,4\}$ and $J=\{2,3,4,5\}$. As generators of the strands algebra $\mathcal{B}_{n, d}$, the strand diagrams 

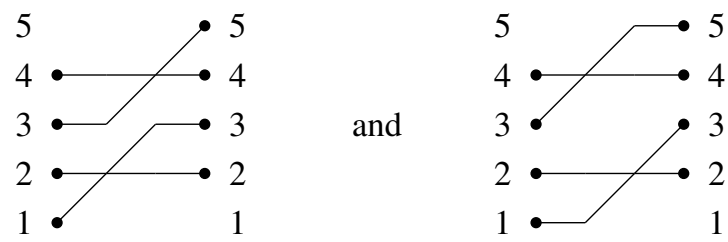

are negatives of each other.

Example 3.1.17. Let $n=5$ and $d=3$. Let $I=\{1,2,3\}, J=\{2,3,4\}$ and $K=\{3,4,5\}$. Consider the following composite of strand diagrams $I \rightarrow J$ and $J \rightarrow K$ :
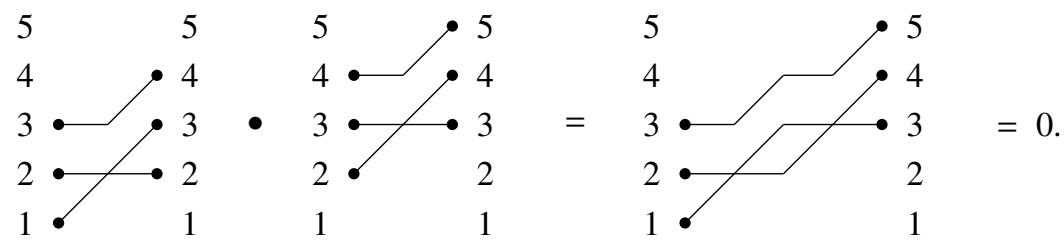

Since concatenating the two strand diagrams results in a strand diagram in which two strands cross more than once, their product vanishes in the strands algebra $\mathcal{B}_{n, d}$.

Example 3.1.18. Let $n=5$ and $d=3$. Let $I=\{1,2,3\}$ and $J=\{3,4,5\}$. We exemplify the action of the differential on a strand diagram from $I$ to $J$ :
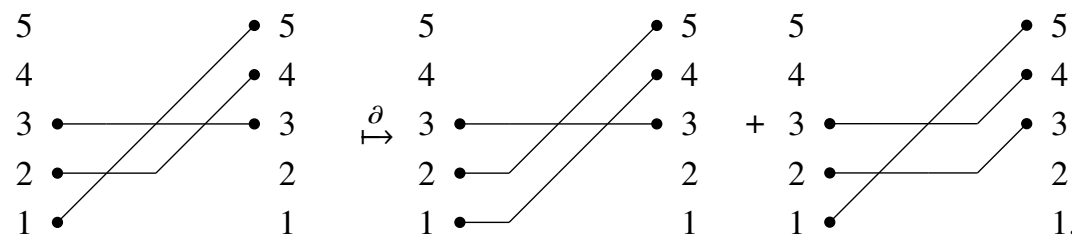

Notice that resolving the second crossing would result in a strand diagram in which two strands cross more than once; therefore, this resolution does not contribute to the differential.

The following observation is crucial for our purposes:

Proposition 3.1.19. Let $I, J \in\left(\begin{array}{l}\mathbf{n} \\ d\end{array}\right)$ be such that $I \leq J$. For each permutation $\pi \in\left[e, \pi_{0}^{J I}\right]$, choose a strand diagram $\varphi=\varphi_{\pi}: I \rightarrow J$ such that $\pi_{\varphi}=\pi$. The set $\left\{\varphi_{\pi} \mid \pi \in\left[e, \pi_{0}^{J I}\right]\right\}$ is a free $\mathbf{k}$-basis of the graded $\mathbf{k}$-module hom $(I, J)$. In particular, $\operatorname{hom}(I, J)$ is isomorphic to the cochain complex $C\left[e, \pi_{0}^{J I}\right]$.

Proof. The proof of [Kho14, Lemma 1] applies verbatim. The second claim follows from Proposition 3.1.23, taking into account that the signs in the definition of the differential on $\operatorname{hom}(I, J)$ yield a balanced signature on the Hasse quiver of the Bruhat interval $\left[e, \pi_{0}^{J I}\right]$.

We also record the following elementary observation:

Lemma 3.1.20. There is a unique differential on the underlying graded $\mathbf{k}$-algebra of the strands algebra $\mathcal{B}_{n, d}$ which endows it with the structure of a differential graded $\mathbf{k}$-algebra, subject to the additional restriction that $\partial(\varphi)=e_{J I}$ for every strand diagram $\varphi: I \rightarrow J$ with a single crossing.

Proof. By construction, the strands algebra $\mathcal{B}_{n, d}$ is generated as a graded $\mathbf{k}$-algebra in cohomological degrees 0 and -1 (since every reduced word is, by definition, a product of simple transpositions). The graded Leibniz rule implies that the differential on $\mathcal{B}_{n, d}$ is completely determined by its action on the generators of these cohomological degrees. The claim follows. 
3.1.3. The quasi-isomorphism $\mathcal{B}_{n, d} \simeq A_{n, d}$

The following theorem is the first step in establishing the quasi-equivalence between the partially wrapped Fukaya category $\mathcal{W}_{n}^{(d)}$ and the perfect derived category $\operatorname{perf}\left(A_{n, d}\right)$ :

Theorem 3.1.21. There is a quasi-isomorphism of differential graded $\mathbf{k}$-algebras

$$
\mathcal{B}_{n, d} \stackrel{\simeq}{\longrightarrow} H^{0}\left(\mathcal{B}_{n, d}\right)
$$

and an isomorphism of (ungraded) $\mathbf{k}$-algebras $H^{0}\left(\mathcal{B}_{n, d}\right) \cong A_{n, d}$.

Remark 3.1.22. When working in characteristic 2 , the cohomology of the strands algebra $\mathcal{B}_{n, d}$ is computed additively in [LOT15, Section 4.1]. However, the authors do not relate the cohomology algebra of the strands algebra with the higher Auslander algebra $A_{n, d}$.

Theorem 3.1.21 is a consequence of the following results:

Proposition 3.1.23. Let $I, J \in\left(\begin{array}{l}\mathbf{n} \\ d\end{array}\right)$ be such that $I \leq J$ and let $\left[e, \pi_{0}^{J I}\right]$ be the corresponding Bruhat interval. There are isomorphisms of graded $\mathbf{k}$-modules

$$
H^{*}(\operatorname{hom}(I, J)) \cong \begin{cases}\mathbf{k}(0) & \text { if } \pi_{0}^{J I}=e, \\ 0 & \text { otherwise. }\end{cases}
$$

In particular, the differential graded $\mathbf{k}$-algebra $\mathcal{B}_{n, d}$ has its cohomology concentrated in degree 0 :

$$
H^{*}\left(\mathcal{B}_{n, d}\right)=H^{0}\left(\mathcal{B}_{n, d}\right)
$$

Proof. Let $I, J \in\left(\begin{array}{l}\mathbf{n} \\ d\end{array}\right)$. By Proposition 3.1.19 the cochain complex $\operatorname{hom}(I, J)$ is isomorphic to $C\left[e, \pi_{0}^{J I}\right]$. By Proposition 3.1.23 the latter complex is acyclic if the permutation $\pi_{0}^{J I}$ is nontrivial and is otherwise isomorphic to the ground ring $\mathbf{k}$ placed in cohomological degree 0 . The claim follows.

Remark 3.1.24. The analogue in characteristic 2 of Proposition 3.1.23 is proven in [LOT15, Proposition 4.2], where, in the case $\pi_{0}^{J I} \neq e$, the authors construct an explicit null-homotopy of the identity morphism of the cochain complex hom $(I, J)$. In order to avoid the sign considerations involved in constructing an explicit null-homotopy in arbitrary characteristic, we have opted for the alternative approach hinted at in [LOT15, Remark 4.4].

Proposition 3.1.25. There is an isomorphism of (ungraded) $\mathbf{k}$-algebras

$$
H^{0}\left(\mathcal{B}_{n, d}\right) \cong A_{n, d}
$$

Proof. Let $I, J \in\left(\begin{array}{l}\mathbf{n} \\ d\end{array}\right)$. By construction, there is an isomorphism of graded $\mathbf{k}$-modules

$$
\mathcal{B}_{n, d}^{0}=\bigoplus_{I, J} \operatorname{hom}^{0}(I, J) \cong \bigoplus_{I \leq J} \mathbf{k} \cdot e_{J I},
$$

where $e_{J I} \in\left[e, \pi_{0}^{J I}\right]$ is the trivial permutation. Notice that the right-hand side can be identified with the underlying k-module of the incidence k-algebra of the poset $\left(\begin{array}{c}\mathbf{n} \\ d\end{array}\right)$. Comparing the multiplication laws on both sides, we conclude that the isomorphism is in fact an isomorphism of (ungraded) k-algebras.

Let $I, J \in\left(\begin{array}{l}\mathbf{n} \\ d\end{array}\right)$ be such that $I \leq J$ and let $e_{J I} \in\left[e, \pi_{0}^{J I}\right]$ be the trivial permutation (which we identify with the unique equivalence class of strand diagrams $I \rightarrow J$ with no crossings). Suppose that there exists an index $1 \leq a<d$ such that $j_{a} \geq i_{a+1}$. We claim that $e_{J I}=0$ in $H^{0}\left(\mathcal{B}_{n, d}\right)$ in this case. Indeed, the simple transposition $s_{a}=(a+1, a)$ lies in $\left[e, \pi_{0}^{J I}\right]$, since

$$
i_{a} \leq j_{a}<j_{a+1}=j_{s_{a}(a)} \quad \text { and } \quad i_{a+1} \leq j_{a}=j_{s_{a}(a+1)},
$$


where the inequalities on the left-hand side hold by definition and the inequality on the right-hand side holds by assumption. Clearly, $\partial(\varphi)=e_{J I}$ for any strand diagram $\varphi$ such that $\pi_{\varphi}=s_{a}$. The claim follows.

This argument shows that the given isomorphism of (ungraded) k-algebras between $\mathcal{B}_{n, d}^{0}$ and $\bigoplus_{I \leq J} \mathbf{k} \cdot e_{J I}$ maps the image of the differential on the left-hand side to the submodule

$$
\left\langle e_{J I} \mid \exists a: j_{a} \geq i_{a+1}\right\rangle
$$

on the right-hand side. Comparing with the definition of the higher Auslander algebra $A_{n, d}$, the claim follows.

We now give the proof of Theorem 3.1.21:

Proof of Theorem 3.1.21. According to Proposition 3.1.23, the differential graded algebra $\mathcal{B}_{n, d}$ has its cohomology concentrated in degree 0 . This immediately implies that the canonical morphism

$$
\mathcal{B}_{n, d} \stackrel{\simeq}{\longrightarrow} H^{0}\left(\mathcal{B}_{n, d}\right)
$$

is a quasi-isomorphism. Finally, Proposition 3.1.25 shows that there is an isomorphism of (ungraded) k-algebras $H^{0}\left(\mathcal{B}_{n, d}\right) \cong A_{n, d}$.

\subsection{The quasi-equivalence $\mathcal{W}_{n}^{(d)} \simeq \operatorname{perf}\left(A_{n, d}\right)$}

Notation 3.2.1. For $1 \leq i \leq n$, we introduce the notation

$$
L_{i}:=L_{0 i} .
$$

More generally, for $I \in\left(\begin{array}{l}\mathbf{n} \\ d\end{array}\right)$ we introduce the Lagrangian

$$
L_{I}:=\prod_{a=1}^{d} L_{i_{a}}=\prod_{a=1}^{d} L_{0 i_{a}}
$$

in $\operatorname{Sym}^{d}\left(\mathbb{D} \backslash \Lambda_{n}\right)$ and define

$$
\mathcal{A}_{n, d}:=\bigoplus_{I, J} \operatorname{hom}\left(L_{I}, L_{J}\right)
$$

(the precise grading structures on these Lagrangians are determined in Proposition 3.2.6); we remind the reader that $\mathcal{A}_{n, d}$ is a differential graded k-algebra - that is, the higher products vanish (see Section 2.1.6).

The following proposition is an immediate consequence of Theorem 2.1.2:

Proposition 3.2.2. The collection $\left\{L_{I} \mid I \in\left(\begin{array}{c}\mathbf{n} \\ d\end{array}\right)\right\}$ generates the partially wrapped Fukaya category $\mathcal{W}_{n}^{(d)}$ as an idempotent-complete triangulated $A_{\infty}$-category. In particular, there is a quasi-equivalence of triangulated $A_{\infty}$-categories

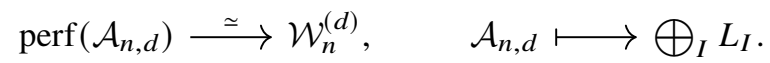

In this section we establish the following quasi-equivalence:

Theorem 3.2.3. Let $n \geq d \geq 1$. There is a quasi-isomorphism of differential graded $\mathbf{k}$-algebras

$$
\mathcal{A}_{n, d} \stackrel{\simeq}{\longrightarrow} A_{n, d} \text {. }
$$

Thus, there exists a quasi-equivalence of triangulated $A_{\infty}$-categories

$$
\operatorname{perf}\left(A_{n, d}\right) \stackrel{\simeq}{\longrightarrow} \mathcal{W}_{n}^{(d)}, \quad A_{n, d} \longmapsto \bigoplus_{I} L_{I} .
$$

Corollary 3.2.4. The differential graded $\mathbf{k}$-algebras and $\mathcal{A}_{n, d}$ and $\mathcal{B}_{n, d}$ are related by a zigzag of quasi-isomorphisms

$$
\mathcal{A}_{n, d} \stackrel{\simeq}{\longrightarrow} A_{n, d} \stackrel{\simeq}{\simeq} \mathcal{B}_{n, d} .
$$


Proof. This is an immediate consequence of Theorems 3.1.21 and 3.2.3.

\subsubsection{Grading structures}

Our first task toward the proof of Theorem 3.2.3 consists of constructing suitable grading structures on the Lagrangians $\left\{L_{I} \mid I \in\left(\begin{array}{l}\mathbf{n} \\ d\end{array}\right)\right\}$.

Proposition 3.2.5. Let $e \in \mathfrak{S}_{d}$ be the trivial permutation. The following statements hold:

1. Let $I, J \in\left(\begin{array}{l}\mathbf{n} \\ d\end{array}\right)$. There are isomorphisms of (ungraded) $\mathbf{k}$-modules

$$
\operatorname{hom}\left(L_{I}, L_{J}\right)^{e} \cong \begin{cases}\mathbf{k} \cdot f_{J I} & \text { if } I \leq J \\ 0 & \text { otherwise. }\end{cases}
$$

2. There is an isomorphism of (ungraded) $\mathbf{k}$-algebras between

$$
\mathcal{A}_{n, d}^{0}=\bigoplus_{I, J} \operatorname{hom}\left(L_{I}, L_{J}\right)^{e}
$$

and the incidence $\mathbf{k}$-algebra of the poset $\left(\begin{array}{l}\mathbf{n} \\ d\end{array}\right)$.

Proof. Recall that

$$
\operatorname{hom}\left(L_{I}, L_{J}\right)^{e}=\operatorname{hom}\left(L_{i_{1}}, L_{j_{1}}\right) \otimes \cdots \otimes \operatorname{hom}\left(L_{i_{d}}, L_{j_{d}}\right)
$$

Statement 1 follows immediately from formula (2.3.1), since the condition that $i_{a} \leq j_{a}$ for all $1 \leq a \leq d$ is precisely the condition that $I \leq J$ in the poset $\left(\begin{array}{l}\mathbf{n} \\ d\end{array}\right)$.

We now prove statement 2 . The isomorphism of (ungraded) $\mathbf{k}$-modules

$$
\mathcal{A}_{n, d}^{0} \cong \bigoplus_{I \leq J} \mathbf{k} \cdot f_{J I}
$$

induces a product operation on the right-hand side of the form

$$
f_{K J} \circ f_{J I}=\varepsilon_{K I}^{J} f_{K I},
$$

where the sign $\varepsilon_{K I}^{J}= \pm 1$ is in general difficult to determine, as it is induced by the orientation of certain moduli spaces of holomorphic disks. We claim that by possibly replacing some of the generators with their negatives, we can assume that all these signs are positive (note that this immediately proves statement 2). We proceed inductively. For $d=1$ and $n \geq d$, the poset $\left(\begin{array}{l}\mathbf{n} \\ d\end{array}\right)$ is isomorphic to a chain with $n$ elements. It is clear that we can inductively choose signs on the generators $f_{i+1, i}:\{i\} \rightarrow\{i+1\}$ to ensure that all signs are positive in this case. Let now $d>1$ and suppose that we have proven the claim for all $d-1$ and $n \geq d-1$. Observe first that $\left(\begin{array}{c}\mathbf{n}-\mathbf{1} \\ d\end{array}\right)$ is a convex subset of $\left(\begin{array}{c}\mathbf{n} \\ d\end{array}\right)$ - that is, a subset closed under the passage to closed intervals. Similarly, the map $I^{\prime} \mapsto I^{\prime} \cup\{n\}$ induces an isomorphism between $\left(\begin{array}{c}\mathbf{n}-\mathbf{1} \\ d-1\end{array}\right)$ and the convex subset

$$
\left(\begin{array}{l}
\mathbf{n} \\
d
\end{array}\right)_{n}=\left\{I \in\left(\begin{array}{l}
\mathbf{n} \\
d
\end{array}\right) \mid n \in I\right\}
$$

of $\left(\begin{array}{l}\mathbf{n} \\ d\end{array}\right)$. Thus, by induction we can assume that the product of generators indexed by elements of either of these subsets involves only positive signs (where we only allow for products of generators indexed by elements of the same convex subset). Let

$$
I_{\min }=\{1, \ldots, d\} \quad \text { and } \quad K_{\max }=\{n-d+1, \ldots, n-1, n\}
$$

be the minimal and the maximal elements in $\left(\begin{array}{l}\mathbf{n} \\ d\end{array}\right)$, respectively. For each pair $J<K$ such that $J \in\left(\begin{array}{c}\mathbf{n}-\mathbf{1} \\ d\end{array}\right)$ and $K \in\left(\begin{array}{c}\mathbf{n} \\ d\end{array}\right)_{n}$, replacing the generator $f_{K J}$ with its negative if necessary, we can assume that

$$
f_{K J} \circ f_{J I_{\min }}=f_{K I_{\min }}
$$

(note that if $J=I_{\min }$ this imposes no condition on the generator $f_{K J}=f_{K I_{\min }}$ ). Let $J<K$ be as before and $I \leq J$ (so in particular $I \in\left(\begin{array}{c}\mathbf{n}-\mathbf{1} \\ d\end{array}\right)$ ); we claim that

$$
f_{K J} \circ f_{J I}=f_{K I} .
$$


Indeed,

$$
\begin{aligned}
f_{K I_{\min }} & =f_{K J} \circ f_{J I_{\min }} \\
& =f_{K J} \circ\left(f_{J I} \circ f_{I I_{\min }}\right) \\
& =\left(f_{K J} \circ f_{J I}\right) \circ f_{I I_{\min }} \\
& =\varepsilon_{K I}^{J} f_{K I} \circ f_{I I_{\min }}=\varepsilon_{K I}^{J} f_{K I_{\min }},
\end{aligned}
$$

and therefore $\varepsilon_{K I}^{J}=1$. Now, for each $K \in\left(\begin{array}{l}\mathbf{n} \\ d\end{array}\right)_{n}$, replacing the generator $f_{K I_{\min }}$ with its negative if necessary, we can assume that

$$
f_{K_{\max } K} \circ f_{K I_{\min }}=f_{K_{\max } I_{\min }} .
$$

Let $K \leq K^{\prime}$ (so in particular $K^{\prime} \in\left(\begin{array}{l}\mathbf{n} \\ d\end{array}\right)_{n}$ ); we claim that

$$
f_{K^{\prime} K} \circ f_{K I_{\min }}=f_{K^{\prime} I_{\min }} .
$$

Indeed,

$$
\begin{aligned}
& f_{K_{\max } I_{\min }}=f_{K_{\max } K} \circ f_{K I_{\min }} \\
& =\left(f_{K_{\max } K^{\prime}} \circ f_{K^{\prime} K}\right) \circ f_{K I_{\min }} \\
& =f_{K_{\max } K^{\prime}} \circ\left(f_{K^{\prime} K} \circ f_{K I_{\min }}\right) \\
& =\varepsilon_{K^{\prime} I_{\min }}^{K} f_{K_{\max } K^{\prime}} \circ f_{K^{\prime} I_{\min }}=\varepsilon_{K^{\prime} I_{\min }}^{K} f_{K_{\max } I_{\min }},
\end{aligned}
$$

and therefore $\varepsilon_{K^{\prime} I_{\min }}^{K}=1$. We can then proceed similarly as before to show that

$$
f_{K^{\prime} K} \circ f_{K J}=f_{K^{\prime} J}
$$

for all $J<K \leq K^{\prime}$ such that $J \in\left(\begin{array}{c}\mathbf{n}-\mathbf{1} \\ d-1\end{array}\right)$ and $K, K^{\prime} \in\left(\begin{array}{l}\mathbf{n} \\ d\end{array}\right)_{n}$, which is what we needed to show. This proves the claim for all $n \geq d$, and by induction on $d$, the general case follows.

Proposition 3.2.6. Let $e \in \mathfrak{S}_{d}$ be the trivial permutation. Up to simultaneous shift, there exist unique grading structures on the Lagrangians $\left\{L_{I} \mid I \in\left(\begin{array}{l}\mathbf{n} \\ d\end{array}\right)\right\}$ such that the graded $\mathbf{k}$-algebra

$$
\bigoplus_{I, J} \operatorname{hom}\left(L_{I}, L_{J}\right)^{e}=\bigoplus_{I \leq J} \operatorname{hom}\left(L_{I}, L_{J}\right)^{e}
$$

is concentrated in degree 0 (for an explanation of the notation $\bigoplus_{I, J} \operatorname{hom}\left(L_{I}, L_{J}\right)^{e}$, see equation (2.1.2)).

Proof. According to Proposition 3.2.5, there is an isomorphism of (ungraded) k-algebras between $\bigoplus_{I, J} \operatorname{hom}\left(L_{I}, L_{J}\right)^{e}$ and the incidence $\mathbf{k}$-algebra of the poset $\left(\begin{array}{c}\mathbf{n} \\ d\end{array}\right)$. In particular, for subsets $I \leq J \leq K$ in $\left(\begin{array}{l}\mathbf{n} \\ d\end{array}\right)$, the composition map

$$
\operatorname{hom}\left(L_{K}, L_{J}\right)^{e} \otimes \operatorname{hom}\left(L_{I}, L_{J}\right)^{e} \stackrel{\simeq}{\longrightarrow} \operatorname{hom}\left(L_{I}, L_{K}\right)^{e}
$$

is an isomorphism of graded k-modules (after choosing arbitrary grading structures on the corresponding Lagrangians).

Fix an arbitrary grading structure on the Lagrangian $L_{1 \ldots d}$. For $I \in\left(\begin{array}{c}\mathbf{n} \\ d\end{array}\right)$ equip the Lagrangian $L_{I}$ with the unique grading structure such that the graded $\mathbf{k}$-module

$$
\operatorname{hom}\left(L_{1 \cdots d}, L_{I}\right) \cong \mathbf{k}
$$

is concentrated in degree 0 (notice that $\{1, \ldots, d\}$ is the smallest element in the poset $\left(\begin{array}{l}\mathbf{n} \\ d\end{array}\right)$ ). We claim that this choice of grading structures has the desired property. Indeed, if $J \in\left(\begin{array}{l}\mathbf{n} \\ d\end{array}\right)$ is such that $J \leq I$, then the composition map

$$
\operatorname{hom}\left(L_{J}, L_{I}\right)^{e} \otimes \operatorname{hom}\left(L_{1 \ldots d}, L_{J}\right)^{e} \stackrel{\simeq}{\longrightarrow} \operatorname{hom}\left(L_{1 \ldots d}, L_{I}\right)^{e}
$$


is an isomorphism of graded k-modules. Since, by construction, the graded k-modules

$$
\operatorname{hom}\left(L_{1 \cdots d}, L_{J}\right)^{e} \quad \text { and } \operatorname{hom}\left(L_{1 \cdots d}, L_{I}\right)^{e}
$$

are concentrated in degree 0 , the graded $\mathbf{k}$-module hom $\left(L_{J}, L_{I}\right)^{e}$ must be concentrated in degree 0 as well. Since the shift functor on $\mathcal{W}_{n}^{(d)}$ induces a free and transitive action on the set of grading structures on the Lagrangian $L_{1 \ldots d}$, the argument also shows that up to global shift, there is a unique choice of grading structures with the required property.

\subsubsection{The proof of Theorem 3.2 .3}

Notation 3.2.7. Once and for all, we fix grading structures on the Lagrangians $\left\{L_{I} \mid I \in\left(\begin{array}{c}\mathbf{n} \\ d\end{array}\right)\right\}$ as in Proposition 3.2.6.

Proposition 3.2.8. Let $I, J \in\left(\begin{array}{l}\mathbf{n} \\ d\end{array}\right)$. There are isomorphisms of graded $\mathbf{k}$-modules

$$
\operatorname{hom}\left(L_{I}, L_{J}\right) \cong \begin{cases}\operatorname{hom}(I, J) & \text { if } I \leq J \\ 0 & \text { otherwise }\end{cases}
$$

In particular, the differential graded $\mathbf{k}$-algebra $\mathcal{A}_{n, d}$ has its cohomology concentrated in degree $0:$

$$
H^{*}\left(\mathcal{A}_{n, d}\right)=H^{0}\left(\mathcal{A}_{n, d}\right)
$$

Proof. By formula (2.3.1) and Proposition 3.1.19, there is an isomorphism of (ungraded) k-modules

$$
\bigoplus_{\pi \in \subseteq_{d}} \operatorname{hom}\left(L_{I}, L_{J}\right)^{\pi}=\bigoplus_{\pi \in\left[e, \pi_{0}^{J I}\right]} \operatorname{hom}\left(L_{I}, L_{J}\right)^{\pi} \cong \bigoplus_{\pi \in\left[e, \pi_{0}^{J I}\right]} \mathbf{k} \cdot \pi
$$

Moreover, since the Lagrangians $\left\{L_{I} \mid I \in\left(\begin{array}{l}\mathbf{n} \\ d\end{array}\right)\right\}$ all have an endpoint in the same boundary component, the results in [Aur10b] show that the differential on a morphism $f \in \operatorname{hom}\left(L_{I}, L_{J}\right)$ lies in the k-module

$$
\bigoplus_{\operatorname{inv}(\pi)=\operatorname{inv}\left(\pi^{\prime}\right)+1} \operatorname{hom}\left(L_{I}, L_{J}\right)^{\pi^{\prime}} .
$$

Since, by Proposition 3.2.6, the graded $\mathbf{k}$-module hom $\left(L_{I}, L_{J}\right)^{e}$ is concentrated in degree 0 , arguing by induction on the number of inversions of $\pi$ (and taking into account that the differential is a morphism of degree -1$)$ we conclude that $\operatorname{hom}\left(L_{I}, L_{J}\right)^{\pi}$ is concentrated in degree $-\operatorname{inv}(\pi)$. Thus, the isomorphism can be promoted to an isomorphism of graded k-modules. The claim then follows, since the Bruhat interval $\left[e, \pi_{0}^{J I}\right]$ is nonempty if and only if $I \leq J$ (see Lemma 3.1.4). The proof of Proposition 3.1.23 applies verbatim to show that $\mathcal{A}_{n, d}$ has its cohomology concentrated in degree 0 .

We now give the proof of Theorem 3.2.3:

Proof of Theorem 3.2.3. By Proposition 3.2.8, the differential graded k-algebra $\mathcal{A}_{n, d}$ has its cohomology concentrated in degree 0 :

$$
H^{*}\left(\mathcal{A}_{n, d}\right) \cong H^{0}\left(\mathcal{A}_{n, d}\right)
$$

In particular, the canonical map

$$
\mathcal{A}_{n, d} \stackrel{\simeq}{\longrightarrow} H^{0}\left(\mathcal{A}_{n, d}\right)
$$

is a quasi-isomorphism. By Proposition 3.2.5 there is an isomorphism of ungraded $\mathbf{k}$-algebras between $\mathcal{A}_{n, d}^{0}$ and the incidence k-algebra of the poset $\left(\begin{array}{l}\mathbf{n} \\ d\end{array}\right)$. The same argument used in the proof of Proposition 3.1.25 then shows that there is an isomorphism of ungraded $\mathbf{k}$-algebras between $H^{0}\left(\mathcal{A}_{n, d}\right)$ and the 
higher Auslander algebra $A_{n, d}$. The existence of the required quasi-equivalence

$$
\operatorname{perf}\left(A_{n, d}\right) \stackrel{\simeq}{\longrightarrow} \mathcal{W}_{n}^{(d)}, \quad A_{n, d} \longmapsto \bigoplus_{I} L_{I},
$$

follows from Proposition 3.2.2.

\subsection{The quasi-equivalence $\mathcal{W}_{n}^{(d)} \simeq \operatorname{perf}\left(A_{n, d}^{\vee}\right)$}

Notation 3.3.1. For $1 \leq i \leq n$ we introduce the notation

$$
L_{i}^{\vee}:=L_{i-1, i}
$$

More generally, for $I \in\left(\begin{array}{c}\mathbf{n} \\ d\end{array}\right)$ we introduce the Lagrangian

$$
L_{I}^{\vee}:=\prod_{a=1}^{d} L_{i_{a}}^{\vee}=\prod_{a=1}^{d} L_{i_{a}-1, i_{a}}
$$

in $\operatorname{Sym}^{d}\left(\mathbb{D} \backslash \Lambda_{n}\right)$. We equip this Lagrangian with the unique grading structure such that the apparent morphism

$$
L_{I}=\prod_{a=1}^{d} L_{0, i_{a}} \longrightarrow \prod_{a=1}^{d} L_{i_{a}-1, i_{a}}=L_{I}^{\vee}
$$

given by the Reeb chords $L_{0, i_{a}} \rightarrow L_{i_{a}-1, i_{a}}$ induced by the Reeb flow along the boundary component of $\mathbb{D} \backslash \Lambda_{n}$ labelled $i_{a}$, is a morphism of degree 0 . Finally, we define the differential graded $\mathbf{k}$-algebra

$$
\mathcal{A}_{n, d}^{\vee}:=\bigoplus_{J, I} \operatorname{hom}\left(L_{J}^{\vee}, L_{I}^{\vee}\right) .
$$

The following proposition is an immediate consequence of Theorem 2.1.2:

Proposition 3.3.2. The collection $\left\{L_{I}^{\vee} \mid I \in\left(\begin{array}{c}\mathbf{n} \\ d\end{array}\right)\right\}$ generates the partially wrapped Fukaya category $\mathcal{W}_{n}^{(d)}$ as an idempotent-complete triangulated $A_{\infty}$-category. Thus, there exists a quasi-equivalence of triangulated $A_{\infty}$-categories

$$
\operatorname{perf}\left(\mathcal{A}_{n, d}^{\vee}\right) \stackrel{\simeq}{\longrightarrow} \mathcal{W}_{n}^{(d)}, \quad \mathcal{A}_{n, d}^{\vee} \longmapsto \bigoplus_{I} L_{I}^{\vee}
$$

In this section we establish the following equivalences:

Theorem 3.3.3. Let $n \geq d \geq 1$. There is an isomorphism of $A_{\infty}$-algebras $\mathcal{A}_{n, d}^{\vee} \cong A_{n, d}^{\vee}$ and, consequently, a quasi-equivalence of triangulated $A_{\infty}$-categories

$$
\operatorname{perf}\left(A_{n, d}^{\vee}\right) \stackrel{\simeq}{\longrightarrow} \mathcal{W}_{n}^{(d)}, \quad A_{n, d}^{\vee} \longmapsto \bigoplus_{I} L_{I}^{\vee},
$$

where $A_{n, d}^{\vee}$ denotes the Koszul dual of the (augmented) $\mathbf{k}$-algebra $A_{n, d}$. Moreover, there is a commutative diagram

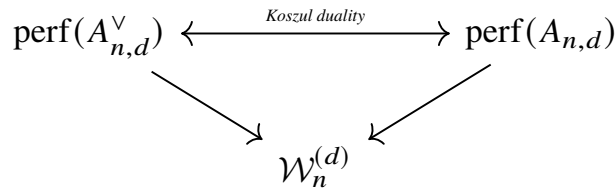

of quasi-equivalences between triangulated $A_{\infty}$-categories. 
Proof. By Theorem 3.2.3 and Propositions 3.3.2 and 3.2.2, it is enough to show that the collections $\left\{L_{I} \mid I \in\left(\begin{array}{c}\mathbf{n} \\ d\end{array}\right)\right\}$ and $\left\{L_{I}^{\vee} \mid I \in\left(\begin{array}{c}\mathbf{n} \\ d\end{array}\right)\right\}$ are Koszul duals of each other; this is the content of Proposition 3.3.10. Indeed, a theorem of Keller concerning the derived Morita equivalence between Koszul dual differential graded $\mathbf{k}$-algebras which are homologically smooth and proper over $\mathbf{k}$ yields the desired quasi-equivalences (see [Ke194, Section 10.5]).

\subsubsection{Standard resolutions in $\mathcal{W}_{n}^{(d)}$}

We recall the existence of certain exact triangles in $\mathcal{W}_{n}^{(d)}$ (see [Aur10b, Lemma 5.2] for details).

Lemma 3.3.4 (Auroux). Let $0 \leq i<j<k \leq n$. The following statements hold (with respect to $a$ suitable choice of grading structures on the corresponding Lagrangians):

1. There is an exact triangle

$$
L_{i j} \rightarrow L_{i k} \rightarrow L_{j k} \rightarrow L_{i j}[1]
$$

in the partially wrapped Fukaya category $\mathcal{W}_{n}^{(1)}$.

2. More generally, let $L$ be the product of $d-1$ pairwise disjoint properly embedded arcs in $\mathbb{D} \backslash \Lambda_{n}$ which are not homotopic to any of the arcs $L_{i j}, L_{i k}, L_{j k}$. There is a nonsplit exact triangle

$$
L \times L_{i j} \rightarrow L \times L_{i k} \rightarrow L \times L_{j k} \rightarrow\left(L \times L_{i j}\right)[1]
$$

in the partially wrapped Fukaya category $\mathcal{W}_{n}^{(d)}$.

3. Suppose that $d \geq 2$. Let $L$ be the product of $d-2$ pairwise disjoint properly embedded arcs in $\mathbb{D} \backslash \Lambda_{n}$ which are not homotopic to any of the $\operatorname{arcs} L_{i j}, L_{i k}, L_{j k}$. There is a quasi-isomorphism

$$
L \times L_{i j} \times L_{i k} \stackrel{\simeq}{\longrightarrow} L \times L_{i j} \times L_{j k}
$$

in the partially wrapped Fukaya category $\mathcal{W}_{n}^{(d)}$.

Lemma 3.3.4 has several useful corollaries. To state them, it is convenient to introduce the following notation:

Notation 3.3.5. Let $\mathbf{n}_{+}=\{0,1, \ldots, n\}$. The map

$$
I \longmapsto I:=\{0\} \cup I
$$

identifies $\left(\begin{array}{l}\mathbf{n} \\ d\end{array}\right)$ with the subset of $\left(\begin{array}{c}\mathbf{n}_{+} \\ d+1\end{array}\right)$ consisting of those $(d+1)$-element subsets $I \subseteq \mathbf{n}_{+}$such that $0 \in I$. For $I=\left\{i_{0}<i_{1}<\cdots<i_{d}\right\}$ in $\left(\begin{array}{c}\mathbf{n}_{+} \\ d+1\end{array}\right)$, we introduce the Lagrangian

$$
L_{I}=\prod_{a=1}^{d} L_{i_{0}, i_{a}}
$$

in $\operatorname{Sym}^{d}\left(\mathbb{D} \backslash \Lambda_{n}^{(d)}\right)$. Thus the set $\left\{L_{I} \mid I \in\left(\begin{array}{l}\mathbf{n} \\ d\end{array}\right)\right\}$ of generators of $\mathcal{W}_{n}^{(d)}$ can be identified with the set

$$
\left\{L_{I} \mid I \in\left(\begin{array}{c}
\mathbf{n}_{+} \\
d+1
\end{array}\right): 0 \in I\right\} .
$$

Finally, for a subset $I \in\left(\begin{array}{c}\mathbf{n}_{+} \\ d+1\end{array}\right)$ such that $0 \notin I$, we introduce the subsets

$$
I_{a}=\{0\} \cup\left(I \backslash\left\{i_{a}\right\}\right), \quad 0 \leq a \leq d,
$$

together with the corresponding Lagrangians $L_{I_{a}}$, which, by construction, belong to the set of generators of $\mathcal{W}_{n}^{(d)}$. 
Corollary 3.3.6. Let $I \in\left(\begin{array}{c}\mathbf{n}_{+} \\ d+1\end{array}\right)$ be such that $0 \notin I$. For each $0 \leq a \leq d$, there is an exact triangle

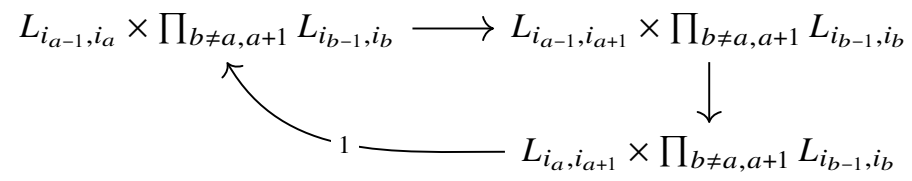

in the partially wrapped Fukaya category $\mathcal{W}_{n}^{(d)}$, where the middle term is quasi-isomorphic to $L_{I_{k}}$.

Proof. This is a special case of Lemma 3.3.4(3.3.4) for the triple $0 \leq i_{a-1}<i_{a}<i_{a+1} \leq n$, where $i_{-1}=0$ by convention.

Corollary 3.3.7. Let $I \in\left(\begin{array}{c}\mathbf{n}_{+} \\ d+1\end{array}\right)$. There is a quasi-isomorphism

$$
L_{I} \stackrel{\simeq}{\longrightarrow} L_{i_{0}, i_{1}} \times L_{i_{1}, i_{2}} \times \cdots \times L_{i_{d-1}, i_{d}}
$$

in the partially wrapped Fukaya category $\mathcal{W}_{n}^{(d)}$. In particular, if $0 \notin I$, there is a quasi-isomorphism

$$
L_{I_{a}} \stackrel{\simeq}{\longrightarrow} L_{i_{a-1}, i_{a+1}} \times \prod_{b \neq k, k+1} L_{i_{b-1}, i_{b}}
$$

in $\mathcal{W}_{n}^{(d)}$ for each $0 \leq a \leq d$.

Proof. The existence of the claimed quasi-isomorphism follows by iterative application of the quasiisomorphisms in Lemma 3.3.4(3.3.4).

Corollary 3.3.8. Fix grading structures on the standard generators of $\mathcal{W}_{n}^{(d)}$ as in Proposition 3.2.6. Let $I \in\left(\begin{array}{c}\mathbf{n}_{+} \\ d+1\end{array}\right)$ be such that $0 \notin I$. Then the object $L_{I}$ of $\mathcal{W}_{n}^{(d)}$ admits a (triangulated) resolution of the form

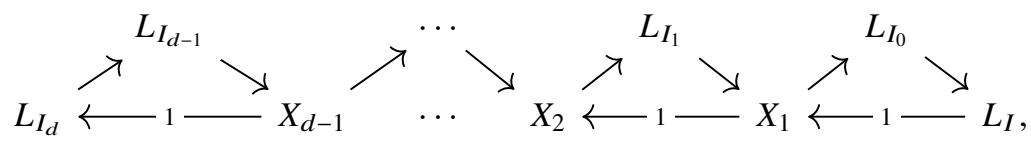

where the grading structure on $L_{I}$ is uniquely determined by the requirement that the morphism $L_{I_{0}} \rightarrow L_{I}$ be of degree 0 .

Proof. The required resolution is obtained by splicing together the exact triangles from Corollary 3.3.6 in the apparent manner. The existence of a unique grading structure on the Lagrangian $L_{I}$ with the desired property is clear because the grading structure on the Lagrangian $L_{I_{0}}$ is already fixed.

\subsubsection{Koszul duality for $\mathcal{W}_{n}^{(d)}$}

Let $\mathcal{A} \rightarrow \bigoplus_{i=1}^{t} \mathbf{k}$ be an augmented differential graded $\mathbf{k}$-algebra. The differential graded $\mathbf{k}$-algebra

$$
\mathcal{A}^{\vee}=\operatorname{hom}_{\mathcal{A}}\left(\bigoplus_{i=1}^{t} \mathbf{k}, \bigoplus_{i=1}^{t} \mathbf{k}\right),
$$

called the Koszul dual of $\mathcal{A}$, admits a canonical augmentation $\mathcal{A}^{\vee} \rightarrow \bigoplus_{i=1}^{t} \mathbf{k}$. Moreover, if $\mathcal{A}$ is proper and homologically smooth over $\mathbf{k}$, then there is an equivalence of differential graded $\mathbf{k}$-categories

$$
\operatorname{perf}(\mathcal{A}) \stackrel{\simeq}{\longrightarrow} \operatorname{perf}\left(\mathcal{A}^{\vee}\right), \quad \bigoplus_{i=1}^{t} \mathbf{k} \longmapsto \mathcal{A}^{\vee},
$$

with quasi-inverse

$$
\operatorname{perf}\left(\mathcal{A}^{\vee}\right) \stackrel{\simeq}{\longrightarrow} \operatorname{perf}(\mathcal{A}), \quad \bigoplus_{i=1}^{t} \mathbf{k} \longmapsto \mathcal{A}
$$

(see [Kel94] for details). 
Lemma 3.3.9. The higher Auslander algebra $A_{n, d}$ is proper and homologically smooth over $\mathbf{k}$.

Proof. The higher Auslander algebra $A_{n, d}$ is clearly proper over $\mathbf{k}$, since its underlying k-module is free of finite rank. To prove that $A_{n, d}$ is homologically smooth over $\mathbf{k}$, one can proceed as follows. First, $A_{n, n} \cong \mathbf{k}$ is certainly homologically smooth over $\mathbf{k}$. Second, we observe that there exists a recollement

$$
\operatorname{perf}\left(A_{n-1, d-1}\right) \underset{i_{R}}{\leftrightarrows} \operatorname{perf}\left(A_{n, d}\right) \stackrel{p_{L}}{\longleftarrow} \operatorname{perf}\left(A_{n-1, d}\right),
$$

which expresses perf $\left(A_{n, d}\right)$ as the upper-triangular gluing of $\operatorname{perf}\left(A_{n-1, d-1}\right)$ and $\operatorname{perf}\left(A_{n-1, d}\right)$ (see, for example, [DJW19, Proposition 2.50]). Inductively, we may assume that the k-algebras $A_{n-1, d-1}$ and $A_{n-1, d}$ are already known to be homologically smooth over $\mathbf{k}$. The claim follows from [LS14], which shows that the gluing of two differential graded $\mathbf{k}$-categories which are homologically smooth over $\mathbf{k}$ is again homologically smooth over $\mathbf{k}$, provided that the gluing bimodule is perfect. To see that the gluing functor

$$
F:=i_{R} \circ p_{L}: \operatorname{perf}\left(A_{n-1, d}\right) \longrightarrow \operatorname{perf}\left(A_{n-1, d-1}\right)
$$

in the recollement is indeed given by a perfect bimodule, consider the induced (homotopy) colimitpreserving functor

$$
\mathbb{L} F_{!}: \mathrm{D}\left(A_{n-1, d}\right) \longrightarrow \mathrm{D}\left(A_{n-1, d-1}\right)
$$

obtained by passing to the Ind-completions of $\operatorname{perf}\left(A_{n-1, d}\right)$ and $\operatorname{perf}\left(A_{n-1, d-1}\right)$, respectively. By construction, the functor $\mathbb{L} F$ ! preserves compact objects, and therefore its underlying bimodule is perfect by [TV07, Lemma 2.8] (keeping in mind that the k-algebra $A_{n-1, d}$ is homologically smooth over $\mathbf{k}$ by the inductive hypothesis).

Theorem 3.3.3 is an immediate consequence of the following statement:

Proposition 3.3.10. The generating collections

$$
\left\{L_{I} \mid I \in\left(\begin{array}{l}
\mathbf{n} \\
d
\end{array}\right)\right\} \quad \text { and } \quad\left\{L_{I}^{\vee} \mid I \in\left(\begin{array}{l}
\mathbf{n} \\
d
\end{array}\right)\right\}
$$

of the partially wrapped Fukaya category $\mathcal{W}_{n}^{(d)}$ are Koszul duals of each other.

Proof. First, note that the k-algebra $A_{n, d}$ has a natural augmentation $A_{n, d} \rightarrow \bigoplus_{I} \mathbf{k} \cdot f_{I I}$ obtained by taking the quotient by its two-sided ideal generated by $\left\{f_{J I}, I<J\right\}$. Lemmas 3.3.11 and 3.3.13 are precisely the homological conditions which characterise the $A_{n, d}$-module $\bigoplus_{I} \mathbf{k} \cdot f_{I I}$ in the perfect derived category perf $\left(A_{n, d}\right)$. Since the higher Auslander algebra $A_{n, d}$ is proper and homologically smooth over $\mathbf{k}$ (see Lemma 3.3.9), the claim follows.

Lemma 3.3.11. Let $I, J \in\left(\begin{array}{l}\mathbf{n} \\ d\end{array}\right)$. There are isomorphisms of graded $\mathbf{k}$-modules

$$
\operatorname{hom}\left(L_{I}, L_{J}^{\vee}\right) \cong \begin{cases}\mathbf{k}(0) & \text { if } I=J \\ 0 & \text { otherwise. }\end{cases}
$$

Proof. Recall that, as (ungraded) k-modules,

$$
\operatorname{hom}\left(L_{I}, L_{J}^{\vee}\right)=\bigoplus_{\pi \in \subseteq_{d}} \operatorname{hom}\left(L_{I}, L_{J}^{\vee}\right)^{\pi},
$$

where

$$
\operatorname{hom}\left(L_{I}, L_{J}^{\vee}\right)^{\pi}=\operatorname{hom}\left(L_{i_{1}}, L_{j_{\pi(1)}}^{\vee}\right) \otimes \operatorname{hom}\left(L_{i_{2}}, L_{j_{\pi(2)}}^{\vee}\right) \otimes \cdots \otimes \operatorname{hom}\left(L_{i_{d}}, L_{j_{\pi(d)}}^{\vee}\right) .
$$




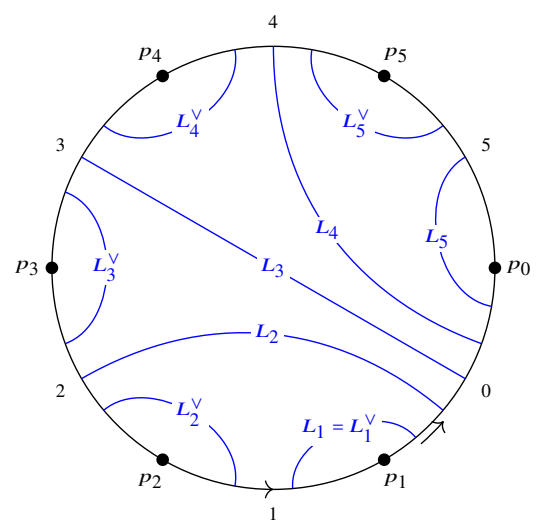

Figure 5. Orthogonality between the generators $\left\{L_{I}\right\}$ and $\left\{L_{J}^{\vee}\right\}$ in the case where $n=5$.

Moreover, there are isomorphisms of (ungraded) k-modules

$$
\operatorname{hom}\left(L_{i}, L_{j}^{\vee}\right) \cong \begin{cases}\mathbf{k} & \text { if } i=j \\ 0 & \text { otherwise }\end{cases}
$$

Indeed, since by definition $L_{i}=L_{0, i}$ and $L_{j}^{\vee}=L_{j-1, j}$, there is an isomorphism $L_{i} \rightarrow L_{j}^{\vee}$ if and only if $i=j$ (note that if $j-1=0$ and $i \leq j$, we must have $i=j=1$ ). It follows readily from the latter isomorphisms that the graded $\mathbf{k}$-module $\operatorname{hom}\left(L_{I}, L_{J}^{\vee}\right)^{\pi}$ is nonzero if and only if $I=J$ and $\pi=e$ is the trivial permutation; see Figure 5 for an illustration in the case where $n=5$. Finally, the grading structure on the Lagrangian $L_{I}^{\vee}$ is, by definition, the unique grading structure such that the graded $\mathbf{k}$-module

$$
\operatorname{hom}\left(L_{I}, L_{I}^{\vee}\right) \cong \mathbf{k}
$$

is concentrated in degree 0 . The claim follows.

We introduce the following auxiliary notation:

Notation 3.3.12. The function $I \mapsto \sum_{a=1}^{d} i_{a}$ endows the poset $\left(\begin{array}{l}\mathbf{n} \\ d\end{array}\right) \subset \mathbb{N}^{d}$ with the structure of a graded poset. For our purposes it is more convenient to consider the (normalised) rank function

$$
\operatorname{rk}(I):=\sum_{a=1}^{d}\left(i_{a}-a\right),
$$

obtained from the usual rank function by subtracting from it the rank of the minimal element $\{1, \ldots, d\}$ of $\left(\begin{array}{l}\mathbf{n} \\ d\end{array}\right)$.

Lemma 3.3.13. Let $I, J \in\left(\begin{array}{l}\mathbf{n} \\ d\end{array}\right)$. There are isomorphisms of graded $\mathbf{k}$-modules

$$
\operatorname{hom}\left(L_{J}^{\vee}, L_{I}^{\vee}\right) \cong \begin{cases}\mathbf{k}(\operatorname{rk}(I)-\operatorname{rk}(J)) & \text { if } \forall a: 0 \leq j_{a}-i_{a} \leq 1, \\ 0 & \text { otherwise. }\end{cases}
$$

In particular, the graded $\mathbf{k}$-algebra

$$
\mathcal{A}_{n, d}^{\vee}=\bigoplus_{J, I} \operatorname{hom}\left(L_{J}^{\vee}, L_{I}^{\vee}\right)
$$

is generated in cohomological degrees 0 and 1 . 


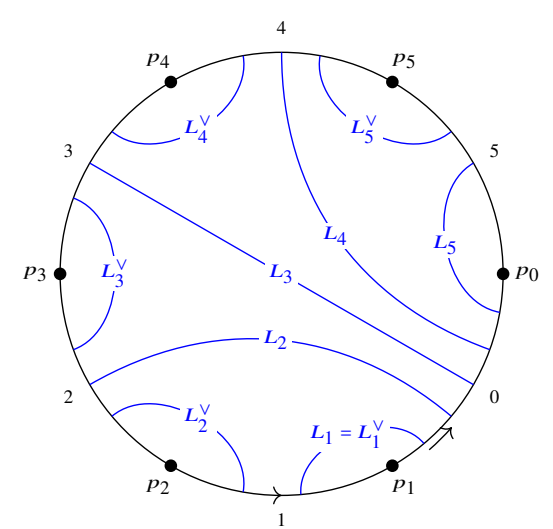

Figure 6. Auxiliary morphisms in the proof of Lemma 3.3.13.

Proof. Recall that, as (ungraded) k-modules,

$$
\operatorname{hom}\left(L_{J}^{\vee}, L_{I}^{\vee}\right)=\bigoplus_{\pi \in \subseteq_{d}} \operatorname{hom}\left(L_{J}^{\vee}, L_{I}^{\vee}\right)^{\pi},
$$

where

$$
\operatorname{hom}\left(L_{J}^{\vee}, L_{I}^{\vee}\right)^{\pi}=\operatorname{hom}\left(L_{j_{1}}^{\vee}, L_{i_{\pi(1)}}^{\vee}\right) \otimes \operatorname{hom}\left(L_{j_{2}}^{\vee}, L_{i_{\pi(2)}}^{\vee}\right) \otimes \cdots \otimes \operatorname{hom}\left(L_{j_{d}}^{\vee}, L_{i_{\pi(d)}}^{\vee}\right) .
$$

Moreover, by formula (2.3.2) there are isomorphisms of (ungraded) k-modules

$$
\operatorname{hom}\left(L_{j}^{\vee}, L_{i}^{\vee}\right) \cong \begin{cases}\mathbf{k} & \text { if } 0 \leq j-i \leq 1, \\ 0 & \text { otherwise. }\end{cases}
$$

It readily follows that there are isomorphisms of (ungraded) k-modules

$$
\operatorname{hom}\left(L_{J}^{\vee}, L_{I}^{\vee}\right) \cong \begin{cases}\mathbf{k} & \text { if } \forall a: 0 \leq j_{a}-i_{a} \leq 1, \\ 0 & \text { otherwise. }\end{cases}
$$

It remains to show that if $0 \leq j_{a}-i_{a} \leq 1$, then the graded k-module hom $\left(L_{J}^{\vee}, L_{I}^{\vee}\right)$ is in fact concentrated in degree $\operatorname{rk}(J)-\operatorname{rk}(I) \geq 0$. We consider first the following special case: there exists an index $i \in I$ such that $J=(I \backslash\{i\}) \cup\{i+1\}$. For simplicity, we set

$$
L=\prod_{k \in I \backslash\{i\}} L_{k}=\prod_{k \in J \backslash\{i+1\}} L_{k}
$$

and

$$
L^{\vee}=\prod_{k \in I \backslash\{i\}} L_{k}^{\vee}=\prod_{k \in J \backslash\{i+1\}} L_{k}^{\vee} .
$$

Consider now the morphism of exact triangles

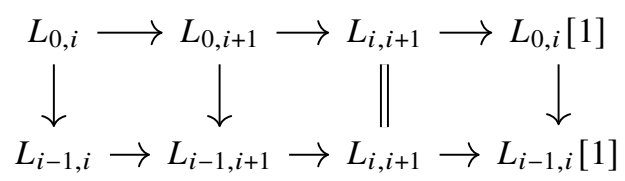

in the partially wrapped Fukaya category $\mathcal{W}_{n}^{(1)}$ (see Figure 6 for an illustration depicting the relevant morphisms). We analyse the induced commutative diagram 


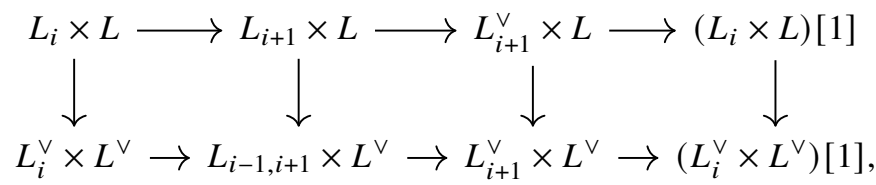

which takes place in the partially wrapped Fukaya category $\mathcal{W}_{n}^{(d)}$. We make the following observations:

- By assumption, the morphism $L_{i} \times L \rightarrow L_{i+1} \times L$ is of degree 0 (see Proposition 3.2.6). In particular, by Lemma 3.3.4 there exists a grading structure on the Lagrangian $L_{i+1}^{\vee} \times L$ such that the top row of the diagram is an exact triangle (with all morphisms of degree 0 ).

- The morphisms

$$
\left(L_{i} \times L\right)[1] \rightarrow\left(L_{i}^{\vee} \times L^{\vee}\right)[1]
$$

and

$$
L_{i+1} \times L \rightarrow L_{i+1}^{\vee} \times L \rightarrow L_{i+1}^{\vee} \times L^{\vee}
$$

are of degree 0 by our choice of grading structures on the Lagrangians

$$
L_{I}^{\vee}=L_{i}^{\vee} \times L^{\vee} \quad \text { and } \quad L_{J}^{\vee}=L_{i+1}^{\vee} \times L^{\vee} .
$$

In particular, the morphism

$$
L_{i+1}^{\vee} \times L \longrightarrow L_{i+1}^{\vee} \times L^{\vee}
$$

is of degree 0 as well.

- We conclude that the bottom row in the commutative square

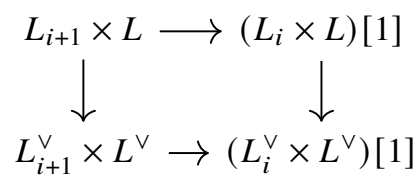

must be given by a morphism of degree 0 , and consequently, the morphism $L_{J}^{\vee} \rightarrow L_{I}^{\vee}$ must be a morphism of degree $1=\operatorname{rk}(J)-\operatorname{rk}(I)$.

This proves the claim in this special case.

We now return to the general case of two subsets $I, J \in\left(\begin{array}{l}\mathbf{n} \\ d\end{array}\right)$ such that the inequality $0 \leq j_{a}-i_{a} \leq 1$ is satisfied for all $1 \leq a \leq d$. Write

$$
J=(I \cap J) \cup\left\{j_{a_{1}}, \ldots, j_{a_{r}}\right\}
$$

where $i_{a_{t}} \in I$ and $j_{a_{t}}-i_{a_{t}}=1$; note that $r=\operatorname{rk}(J)-\operatorname{rk}(I)$. The apparent morphism $L_{J}^{\vee} \rightarrow L_{I}^{\vee}$ factorises as the composite of the $r$ morphisms

$$
\prod_{t=1}^{s} L_{i_{a_{t}}}^{\vee} \times \prod_{t=s+1}^{r} L_{j_{a_{t}}}^{\vee} \times \prod_{k \in I \cap J} L_{k}^{\vee} \longrightarrow \prod_{t=1}^{s-1} L_{i_{a_{t}}}^{\vee} \times \prod_{t=s}^{r} L_{j_{a_{t}}}^{\vee} \times \prod_{k \in I \cap J} L_{k}^{\vee}
$$

indexed by $r \geq s \geq 1$. Since each of these morphisms has degree 1 by the previous argument, the morphism $L_{J}^{\vee} \rightarrow L_{I}^{\vee}$ has degree $r=\operatorname{rk}(J)-\operatorname{rk}(I)$, which is what we needed to prove. 
3.4. The quasi-equivalence $\mathcal{W}_{n}^{(d)} \simeq \operatorname{perf}\left(A_{n, n-d}\right)$

Notation 3.4.1. For $I \in\left(\begin{array}{l}\mathbf{n} \\ d\end{array}\right)$ we introduce the (graded) Lagrangian

$$
L_{I}^{\#}:=L_{I}^{\vee}[-\operatorname{rk}(I)]
$$

in $\operatorname{Sym}^{d}\left(\mathbb{D} \backslash \Lambda_{n}\right)$ and define the differential graded $\mathbf{k}$-algebra

$$
\mathcal{A}_{n, d}^{\#}:=\bigoplus_{J, I} \operatorname{hom}\left(L_{J}^{\sharp}, L_{I}^{\sharp}\right) .
$$

The following proposition is an immediate consequence of Theorem 2.1.2:

Proposition 3.4.2. The collection $\left\{L_{I}^{\sharp} \mid I \in\left(\begin{array}{l}\mathbf{n} \\ d\end{array}\right)\right\}$ generates the partially wrapped Fukaya category $\mathcal{W}_{n}^{(d)}$ as an idempotent-complete triangulated $A_{\infty}$-category. Thus there exists a quasi-equivalence of triangulated $A_{\infty}$-categories

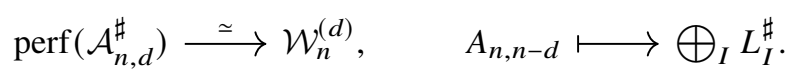

In this section we establish the following quasi-equivalence:

Theorem 3.4.3. Let $n \geq d \geq 1$. There is an isomorphism of differential graded $\mathbf{k}$-algebras $\mathcal{A}_{n, d}^{\sharp} \cong$ $A_{n, n-d}$. Thus there is a quasi-equivalence of triangulated $A_{\infty}$-categories

$$
\operatorname{perf}\left(A_{n, n-d}\right) \stackrel{\simeq}{\longrightarrow} \mathcal{W}_{n}^{(d)}, \quad A_{n, n-d} \longmapsto \bigoplus_{I} L_{I}^{\sharp}
$$

Lemma 3.4.4. The graded $\mathbf{k}$-algebra $\mathcal{A}_{n, d}^{\sharp}$ is concentrated in degree 0 .

Proof. Let $I, J \in\left(\begin{array}{l}\mathbf{n} \\ d\end{array}\right)$. By Lemma 3.3.13, the graded k-module $\operatorname{hom}\left(L_{J}^{\vee}, L_{I}^{\vee}\right)$ either vanishes or is concentrated in degree $\operatorname{rk}(J)-\operatorname{rk}(I)$. Consequently, the graded $\mathbf{k}$-module

$$
\begin{aligned}
\operatorname{hom}\left(L_{J}^{\sharp}, L_{I}^{\sharp}\right) & =\operatorname{hom}\left(L_{J}^{\vee}[-\operatorname{rk}(J)], L_{I}^{\vee}[-\operatorname{rk}(I)]\right) \\
& \cong \operatorname{hom}\left(L_{J}^{\vee}, L_{I}^{\vee}[\operatorname{rk}(J)-\operatorname{rk}(I)]\right) \\
& \cong \operatorname{hom}\left(L_{J}^{\vee}, L_{I}^{\vee}\right)(\operatorname{rk}(J)-\operatorname{rk}(I))
\end{aligned}
$$

is concentrated in degree 0 . The claim follows.

The proof of the following combinatorial statement is straightforward and is left to the reader:

Lemma 3.4.5. Let $n>d \geq 1$ and $I, J \in\left(\begin{array}{l}\mathbf{n} \\ d\end{array}\right)$. We let

$$
I^{\circ}=\mathbf{n} \backslash I=\left\{u_{1}<\cdots<u_{n-d}\right\} \quad \text { and } \quad J^{\circ}=\mathbf{n} \backslash J=\left\{v_{1}<\cdots<v_{n-d}\right\} .
$$

The inequalities $0 \leq j_{a}-i_{a} \leq 1$ are satisfied for all $1 \leq a \leq d$ if and only if $J^{\circ} \leq I^{\circ}$ and $v_{b}<u_{b+1}$ for all $1 \leq b<n-d$.

We are ready to prove Theorem 3.4.3. 
Proof of Theorem 3.4.3. First, by Lemmas 3.4.4 and 3.3.13 there are isomorphisms of k-modules

$$
\operatorname{hom}\left(L_{J}^{\sharp}, L_{I}^{\sharp}\right) \cong \begin{cases}\mathbf{k} \cdot g_{I J} & \text { if } \forall a: 0 \leq j_{a}-i_{a} \leq 1, \\ 0 & \text { otherwise. }\end{cases}
$$

Let

$$
I^{\circ}=\mathbf{n} \backslash I=\left\{u_{1}<\cdots<u_{n-d}\right\} \quad \text { and } \quad J^{\circ}=\mathbf{n} \backslash J=\left\{v_{1}<\cdots<v_{n-d}\right\} .
$$

By Lemma 3.4.5, the condition that $0 \leq j_{a}-i_{a} \leq 1$ for all $1 \leq a \leq d$ is equivalent to the condition that $J^{\circ} \leq I^{\circ}$ and $v_{b}<u_{b+1}$ for all $1 \leq b<n-d$. Keeping in mind the poset anti-isomorphism $\left(\begin{array}{l}\mathbf{n} \\ d\end{array}\right) \rightarrow\left(\begin{array}{c}\mathbf{n} \\ n-d\end{array}\right), I \mapsto I^{\circ}$, we see that the underlying $\mathbf{k}$-modules of the $\mathbf{k}$-algebras $\mathcal{A}_{n, d}^{\sharp}$ and $A_{n, n-d}$ can be identified. Comparing the multiplication laws on both sides, we conclude that there is an isomorphism of k-algebras

$$
\mathcal{A}_{n, d}^{\sharp}=\bigoplus_{J, I} \operatorname{hom}\left(L_{J}^{\sharp}, L_{I}^{\sharp}\right) \cong A_{n, n-d} .
$$

The existence of the required quasi-equivalence follows from Proposition 3.4.2.

\subsection{The Serre functor and Iyama's cluster tilting subcategory of $\mathcal{W}_{n}^{(d)}$}

In this section we give a simple geometric description of the Serre functor on the partially wrapped Fukaya category $\mathcal{W}_{n}^{(d)}$ and use it to describe a distinguished subcategory first investigated by Iyama in the context of higher Auslander-Reiten theory. Throughout this subsection, we assume that $\mathbf{k}$ is a field.

\subsubsection{Geometric description of the Serre functor on $\mathcal{W}_{n}^{(d)}$}

For definiteness, let $\mathbb{D}$ be the 2-dimensional unit disk and $\Lambda_{n}$ be the set of $(n+1)$ th roots of unity. Observe that there is a symplectomorphism $\mathbb{D} \rightarrow \mathbb{D}$, given by counterclockwise rotation by $\frac{2 \pi}{n+1}$, which cyclically permutes the set $\Lambda_{n}$ of stops. Passing to symmetric products, we obtain a symplectomorphism

$$
r: \operatorname{Sym}^{d}(\mathbb{D}) \stackrel{\cong}{\longrightarrow} \operatorname{Sym}^{d}(\mathbb{D})
$$

which preserves $\Lambda_{n}^{(d)}$. To extract an autoequivalence of $\mathcal{W}_{n}^{(d)}$ from $r$ we need to lift it to a graded symplectomorphism; since $H^{1}\left(\operatorname{Sym}^{d}(\mathbb{D})\right)=0$, there is no obstruction to the existence of such a graded lift, and all possible graded lifts of $r$ form a $\mathbb{Z}$-torsor (see [Sei00]). Each graded lift of $r$ induces an autoequivalence

$$
\mathfrak{r}: \mathcal{W}_{n}^{(d)} \simeq \mathcal{W}_{n}^{(d)}
$$

different choices of graded lifts of $r$ induce autoequivalences that differ only by a power of the shift functor on $\mathcal{W}_{n}^{(d)}$. The next proposition shows that a particular graded lift of $r$ gives rise to an autoequivalence $\mathfrak{r}$ of $\mathcal{W}_{n}^{(d)}$ that agrees with its Serre functor on $\mathcal{W}_{n}^{(d)}$ :

Proposition 3.5.1. Let $\mathbf{k}$ be a field. Let $\mathbb{D}$ be the 2-dimensional unit disk and $\Lambda_{n}$ be the set of $(n+1)$ th roots of unity. Let $r: \mathbb{D} \rightarrow \mathbb{D}$ be the symplectomorphism given by counterclockwise rotation by $\frac{2 \pi}{n+1}$. There exists a graded lift of the induced symplectomorphism

$$
r: \operatorname{Sym}^{d}(\mathbb{D}) \stackrel{\cong}{\longrightarrow} \operatorname{Sym}^{d}(\mathbb{D})
$$


such that the induced diagram of $A_{\infty}$-functors

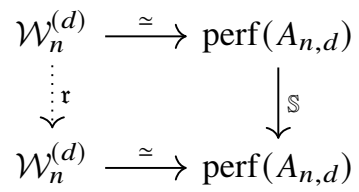

commutes, where

$$
\mathbb{S}=-\otimes_{A_{n, d}}^{\mathbb{L}} \operatorname{Hom}_{\mathbf{k}}\left(A_{n, d}, \mathbf{k}\right): \operatorname{perf}\left(A_{n, d}\right) \stackrel{\simeq}{\longrightarrow} \operatorname{perf}\left(A_{n, d}\right)
$$

is the Serre functor on $\operatorname{perf}\left(A_{n, d}\right)$ and both horizontal quasi-equivalences are the quasi-equivalence from Theorem 3.2.3.

Proof. Let $I \in\left(\begin{array}{c}\mathbf{n}+\mathbf{1} \\ d+1\end{array}\right)$ be such that $0 \in I$ and let $f_{I I}$ be the corresponding idempotent in $A_{n, d}$. The image of the right module $f_{I I} A_{n, d}$ under the composite

$$
\operatorname{perf}\left(A_{n, d}\right) \stackrel{\simeq}{\longrightarrow} \mathcal{W}_{n}^{(d)} \stackrel{\mathrm{r}}{\longrightarrow} \mathcal{W}_{n}^{(d)}
$$

is the object

$$
\mathfrak{r} L_{I}=\prod_{a=1}^{d} r L_{0, i_{a}}=\prod_{a=1}^{d} L_{i_{a}-1, n} .
$$

Iterated application of Lemma 3.3.4 yields a quasi-isomorphism $\mathfrak{r} L_{I} \simeq L_{r I}$, where

$$
r I=\left\{i_{1}-1, \ldots, i_{d}-1, n\right\} \in\left(\begin{array}{c}
\mathbf{n}+\mathbf{1} \\
d+1
\end{array}\right) .
$$

It is straightforward to verify that the standard resolution of the object $L_{r I}$ provided by Corollary 3.3.8 corresponds to the minimal projective resolution of the injective right $A_{n, d}$-module $D\left(A_{n, d} f_{I I}\right)$, as described, for example, in [OT12, Proposition 3.17] or [JK19, Proposition 2.7]. It follows that the restriction of the composite

$$
\operatorname{perf}\left(A_{n, d}\right) \stackrel{\simeq}{\longrightarrow} \mathcal{W}_{n}^{(d)} \stackrel{\mathrm{r}}{\longrightarrow} \mathcal{W}_{n}^{(d)} \stackrel{\simeq}{\longrightarrow} \operatorname{perf}\left(A_{n, d}\right)
$$

to the full subcategory of perf $\left(A_{n, d}\right)$ spanned by the regular representation $A_{n, d}$ agrees with the Serre functor on perf $\left(A_{n, d}\right)$ up to a power of the shift, corresponding to a choice of a graded lift of the symplectomorphism $r: \operatorname{Sym}^{d}(\mathbb{D}) \rightarrow \operatorname{Sym}^{d}(\mathbb{D})$. The claim follows.

Remark 3.5.2. The geometric description of the Serre functor on $\mathcal{W}_{n}^{(d)}$ given in Proposition 3.5 .1 makes it apparent that its $(n+1)$ th iteration must be a power of the shift functor; indeed, the symplectomorphism $r^{n+1}: \mathbb{D} \rightarrow \mathbb{D}$ is the identity. More precisely, there is a quasi-isomorphism

$$
\mathbb{S}^{n+1} \simeq[d(n-d)]
$$

of functors $\mathcal{W}_{n}^{(d)} \rightarrow \mathcal{W}_{n}^{(d)}$ expressing the known fractionally Calabi-Yau property of the quasiequivalent category perf $\left(A_{n, d}\right)$ (see [HI11] for details). Note that this power of the shift is invariant under the passage $d \mapsto n-d$, in agreement with the quasi-equivalence $\mathcal{W}_{n}^{(d)} \simeq \mathcal{W}_{n}^{(n-d)}$ from Theorem 3.3.3.

3.5.2. Geometric description of Iyama's $d$-cluster-tilting subcategory of $\mathcal{W}_{n}^{(d)}$

Notation 3.5.3. For an idempotent complete $A_{\infty}$-category $\mathcal{A}$ admitting finite direct sums and a collection $\mathcal{X}$ of objects of $\mathcal{A}$, we let add $\mathcal{X}$ be the smallest full subcategory of $\mathcal{A}$ which is idempotent complete, is closed under finite direct sums and contains $\mathcal{X}$. 
As explained in the introduction, [Iya11, Theorem 1.18] - one of the main results of that paper shows that the higher Auslander algebra $A_{n, d}$ is a $d$-Auslander algebra in the sense that it satisfies the inequalities

$$
\text { gl. } \operatorname{dim} A_{n, d} \leq d \leq \text { dom. } \operatorname{dim} A_{n, d} \text {. }
$$

As a consequence of the Auslander-Iyama correspondence [Iya07a] and [Iya11, Theorem 1.23], the subcategory

$$
\mathcal{U}\left(A_{n, d}\right):=\operatorname{add}\left\{\mathbb{S}_{d}^{k}\left(A_{n, d}\right) \mid k \in \mathbb{Z}\right\}
$$

is a so-called $d \mathbb{Z}$-cluster-tilting subcategory [Iya07b, IJ17] of the triangulated category $H^{0}\left(\operatorname{perf}\left(A_{n, d}\right)\right)$, where

$$
\mathbb{S}=-\otimes_{A_{n, d}}^{\mathbb{L}} \operatorname{Hom}_{\mathbf{k}}\left(A_{n, d}, \mathbf{k}\right): \operatorname{perf}\left(A_{n, d}\right) \stackrel{\simeq}{\longrightarrow} \operatorname{perf}\left(A_{n, d}\right)
$$

is the Serre functor and $\mathbb{S}_{d}=\mathbb{S}[-d]$. By definition, this means that a perfect complex $X$ lies in $\mathcal{U}\left(A_{n, d}\right)$ if and only if

$$
\forall k \notin d \mathbb{Z} \text { and } \forall M \in \mathcal{U}\left(A_{n, d}\right): \operatorname{Hom}(X, M[k])=0
$$

if and only if

$$
\forall k \notin d \mathbb{Z} \text { and } \forall M \in \mathcal{U}\left(A_{n, d}\right): \operatorname{Hom}(M, X[k])=0 .
$$

This kind of subcategory plays an important role in higher Auslander-Reiten theory and in a higherdimensional version of homological algebra. For example, as shown by Geiss, Keller and Oppermann [GKO13], the additive category $H^{0}\left(\mathcal{U}\left(A_{n, d}\right)\right)$, equipped with the $d$-fold shift functor [d], has the structure of a $(d+2)$-angulated category. Similarly, the subcategory

$$
\mathcal{M}\left(A_{n, d}\right):=\mathcal{U}\left(A_{n, d}\right) \cap \bmod \left(A_{n, d}\right) \subseteq \bmod \left(A_{n, d}\right)
$$

is a $d \mathbb{Z}$-cluster-tilting subcategory of the abelian category $\bmod \left(A_{n, d}\right)$ of finite-dimensional $A_{n, d^{-}}$ modules, which implies that $\mathcal{M}\left(A_{n, d}\right)$ is a $d$-abelian category in the sense of [Jas16]. In some sense, the homological properties of these subcategories can be thought of as 'witnesses' to the higher-dimensional nature of the k-algebra $A_{n, d}$.

Under the quasi-equivalence $\operatorname{perf}\left(A_{n, d}\right) \simeq \mathcal{W}_{n}^{(d)}$ from Theorem 3.2.3, the subcategory $\mathcal{U}\left(A_{n, d}\right)$ of $\operatorname{perf}\left(A_{n, d}\right)$ induces a $d \mathbb{Z}$-cluster-tilting subcategory of the partially wrapped Fukaya category $\mathcal{W}_{n}^{(d)}$ which we describe later.

Proposition 3.5.4. Let $\mathbf{k}$ be a field. Fix grading structures on the Lagrangians $\left\{L_{I} \mid I \in\left(\begin{array}{c}\mathbf{n} \\ d\end{array}\right)\right\}$ as in Proposition 3.2.6. Fix the grading structure on the symplectomorphism $r: \operatorname{Sym}^{d}(\mathbb{D}) \rightarrow \operatorname{Sym}^{d}(\mathbb{D})$ from Proposition 3.5.1 so that the induced autoequivalence

$$
\mathfrak{r}: \mathcal{W}_{n}^{(d)} \simeq \mathcal{W}_{n}^{(d)}
$$

is the Serre functor, and define $\mathfrak{r}_{d}:=\mathfrak{r}[-d]$. The full subcategory

$$
\mathcal{U}_{n}^{(d)}:=\operatorname{add}\left\{\mathrm{r}_{d}^{k}\left(L_{I}\right) \mid I \in\left(\begin{array}{l}
\mathbf{n} \\
d
\end{array}\right), k \in \mathbb{Z}\right\}
$$

of $\mathcal{W}_{n}^{(d)}$ is a dZZ-cluster-tilting subcategory of $H^{0}\left(\mathcal{W}_{n}^{(d)}\right)$. 
Proof. Note that $A_{n, d} \mapsto \bigoplus_{I} L_{I}$ under the quasi-equivalence perf $\left(A_{n, d}\right) \simeq \mathcal{W}_{n}^{(d)}$, from Theorem 3.2.3. Moreover, Proposition 3.5.1 implies the existence of a commutative diagram

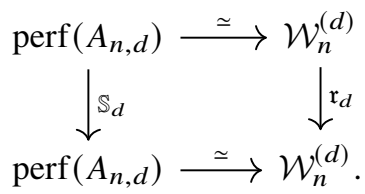

Therefore, $\mathcal{U}\left(A_{d, n}\right)$ corresponds to $\mathcal{U}_{n}^{(d)}$ under the quasi-equivalence $\operatorname{perf}\left(A_{n, d}\right) \simeq \mathcal{W}_{n}^{(d)}$. The claim follows.

Remark 3.5.5. The indecomposable objects in the $d \mathbb{Z}$-cluster-tilting subcategory $\mathcal{U}_{n}^{(d)}$ of $\mathcal{W}_{n}^{(d)}$ are represented by the $d$-fold shifts of the Lagrangians $\left\{L_{I} \mid I \in\left(\begin{array}{c}\mathbf{n}_{+} \\ d+1\end{array}\right)\right\}$ (equipped with grading structures as in Corollary 3.3.8). Since $\mathcal{U}_{n}^{(d)}$ is a $d \mathbb{Z}$-cluster-tilting subcategory of the partially wrapped Fukaya category $\mathcal{W}_{n}^{(d)}$, an object $X$ lies in the $d \mathbb{Z}$-cluster-tilting subcategory $\mathcal{U}_{n}^{(d)}$ if and only if

$$
\forall k \notin d \mathbb{Z} \text { and } \forall L \in \mathcal{U}_{n}^{(d)}: \operatorname{Hom}(X, L[k])=0
$$

if and only if

$$
\forall k \notin d \mathbb{Z} \text { and } \forall L \in \mathcal{U}_{n}^{(d)}: \operatorname{Hom}(L, X[k])=0 .
$$

In particular, given $I, J \in\left(\begin{array}{c}\mathbf{n}+\mathbf{1} \\ d+1\end{array}\right)$, the extension space $\operatorname{Hom}\left(L_{I}, L_{J}[k]\right)$ vanishes for all $k \notin d \mathbb{Z}$.

Remark 3.5.6. Oppermann and Thomas [OT12] provide a beautiful classification of the Miyashita tilting modules [Miy86] which belong to the $d$-cluster-tilting subcategory

$$
\mathcal{M}\left(A_{n, d}\right)=\mathcal{U}\left(A_{n, d}\right) \cap \bmod \left(A_{n, d}\right)
$$

of $\bmod \left(A_{n, d}\right)$ in terms of triangulations of a $2 d$-dimensional cyclic polytope with $n+d+1$ vertices (we remind the reader that $A_{n, d}$ is associated with the Dynkin type $\left.\mathbb{A}_{n-d+1}\right)$; under this bijection, the so-called 'mutation' of tilting modules corresponds to the bistellar flip of triangulations (see [Ram97] or [DLRS10, Section 6.1] for further information on cyclic polytopes and their triangulations). Under the quasiequivalence $\mathcal{W}_{n}^{(d)} \simeq \operatorname{perf}\left(A_{n, d}\right)$ from Theorem 3.2.3, this representation-theoretic procedure provides numerous generators for the partially wrapped Fukaya category $\mathcal{W}_{n}^{(d)}$ whose derived endomorphism algebras have their cohomology concentrated in degree 0 .

Remark 3.5.7. In the foregoing discussion, it is essential that we work over a field rather than over an arbitrary commutative ring. Recall that for each $d \geq 1$, there is a quasi-equivalence

$$
\operatorname{perf}(\mathbf{k}) \simeq \mathcal{W}_{d}^{(d)}
$$

If $\mathbf{k}$ is a field, the triangulated category $H^{0}(\operatorname{perf}(\mathbf{k}))$ has a $d \mathbb{Z}$-cluster-tilting subcategory

$$
\operatorname{add}\{\mathbf{k}[-d k] \mid k \in \mathbb{Z}\} \subseteq \operatorname{perf}(\mathbf{k})
$$

for each $d \geq 1$, where we use the fact that the Serre functor on perf $(\mathbf{k})$ is the identity functor. In contrast, if $\mathbf{k}=\mathbb{Z}$ it is easy to see that $H^{0}(\operatorname{perf}(\mathbb{Z}))$ does not admit a $d \mathbb{Z}$-cluster-tilting subcategory for $d>1$ (a $1 \mathbb{Z}$-cluster-tilting subcategory always exists and coincides with the ambient category).

\subsection{Examples}

We conclude this section with some examples to illustrate our results. 
3.6.1. The quasi-equivalence $\mathcal{W}_{3}^{(1)} \simeq \mathcal{W}_{3}^{(2)}$

For simplicity, we let $\mathbf{k}$ be a field. We illustrate the quasi-equivalence $\mathcal{W}_{n}^{(d)} \simeq \mathcal{W}_{n}^{(n-d)}$ in the simplest nontrivial case: $n=3$ and $d=1$.

The following diagram depicts the so-called Auslander-Reiten quiver [Hap88] of the triangulated category $H^{0}\left(\mathcal{W}_{3}^{(1)}\right)$, where for simplicity we write $i j$ in place of the Lagrangian $L_{i j}$ :

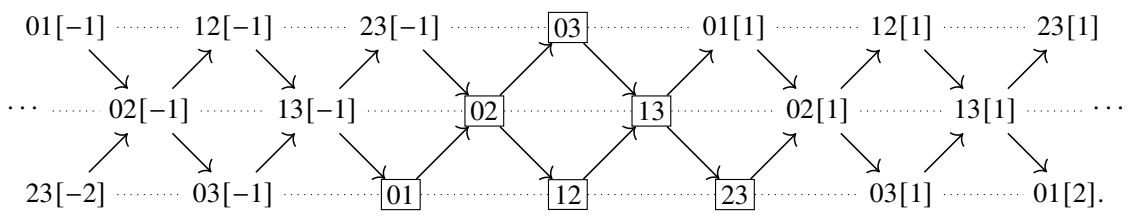

The vertices of the quiver correspond to the indecomposable objects in $H^{0}\left(\mathcal{W}_{3}^{(1)}\right)$; the arrows correspond to a k-basis of the space of irreducible morphisms - that is, the nonisomorphisms which cannot be expressed as a nontrivial composite of nonisomorphisms. The dotted lines indicate the apparent commutativity and zero relations. We have chosen grading structures on the Lagrangians so that all depicted morphisms have degree 0 . The action of the derived Auslander-Reiten translation $\mathbb{S}_{1}=\mathbb{S}[-1]$ is given by left horizontal translation. Finally, we have boxed the six indecomposable objects that belong to the heart of the $t$-structure induced by the quasi-equivalence $\mathcal{W}_{3}^{(1)} \simeq \operatorname{perf}\left(A_{3,1}\right)$; these objects form a complete set of representatives of the isomorphism classes of indecomposable objects in $\mathcal{W}_{3}^{(1)}$ up to the action of the shift functor.

We remind the reader that for $0 \leq i<j<k \leq n$, there is an equivalence $L_{i j} \times L_{i k} \simeq L_{i j} \times L_{j k}$ in $\mathcal{W}_{n}^{(2)}$ induced by the exact triangle

$$
L_{i j} \rightarrow L_{i k} \rightarrow L_{j k} \rightarrow L_{i j}[1]
$$

in the partially wrapped Fukaya category $\mathcal{W}_{n}^{(1)}$ (see Lemma 3.3.4). The following diagram depicts the Auslander-Reiten quiver of the triangulated category $H^{0}\left(\mathcal{W}_{3}^{(2)}\right)$, where for simplicity we write $i j, k \ell$ in place of the Lagrangian $L_{i j} \times L_{k \ell}$ :

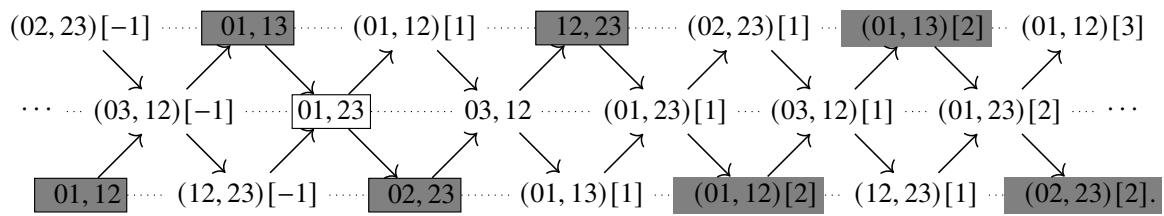

Again, we have chosen grading structures on the Lagrangians so that all depicted morphisms have degree 0 . We have boxed the five indecomposable objects that belong to the heart of the $t$-structure induced by the quasi-equivalence $\mathcal{W}_{3}^{(2)} \simeq \operatorname{perf}\left(A_{3,2}\right)$; together with the Lagrangian $L_{03} \times L_{12}$, these objects form a complete set of representatives of the isomorphism classes of indecomposable objects in $\mathcal{W}_{3}^{(1)}$ up to the action of the shift functor. The indecomposable objects in the $2 \mathbb{Z}$-cluster-tilting subcategory $\mathcal{U}_{3}^{(2)}$ of the triangulated category $H^{0}\left(\mathcal{W}_{3}^{(2)}\right)$ are highlighted; notice that this subcategory is spanned by the (finite direct sums of) even shifts of the four highlighted objects in the heart.

\subsubsection{The $2 \mathbb{Z}$-cluster-tilting subcategory of $H^{0}\left(\mathcal{W}_{4}^{(2)}\right)$}

The following diagram depicts a complete set of representatives of the isomorphism classes of indecomposable objects in the $2 \mathbb{Z}$-cluster-tilting subcategory $\mathcal{U}_{4}^{(2)}$ of the triangulated category $H^{0}\left(\mathcal{W}_{4}^{(2)}\right)$ (see Proposition 3.5.4; all other objects in this subcategory are obtained as even shifts of those depicted): 


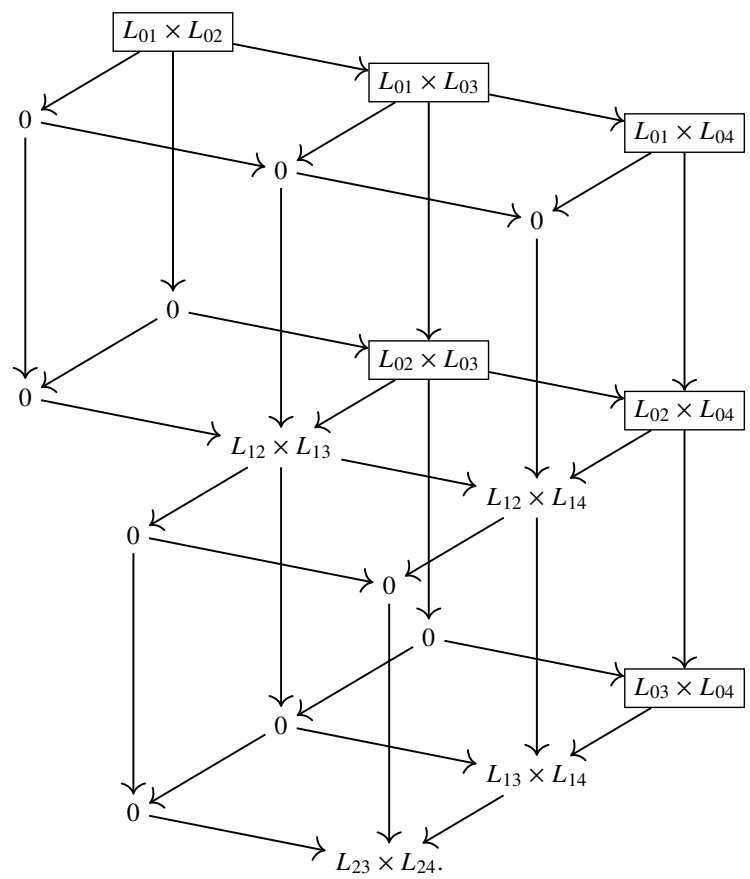

The generators $\left\{L_{I}\right\}$ of the partially wrapped Fukaya category $\mathcal{W}_{4}^{(2)}$ are boxed. In the diagram, all squares commute; note, however, that in the $A_{\infty}$-category $\mathcal{W}_{4}^{(2)}$ there are further higher operations which witness the fact that the apparent rectilinear cubes are bicartesian.

\subsubsection{Explicit examples in the case where $n=5$}

We conclude this section by displaying the endomorphism algebras

$$
H^{*}\left(\mathcal{A}_{n, d}\right) \cong A_{n, d} \quad \text { and } \quad \mathcal{A}_{n, d}^{\#} \cong A_{n, n-d}
$$

in the case where $n=5$ for all $1 \leq d<n$.

We begin with the k-algebras $H^{*}\left(\mathcal{A}_{n, d}\right) \cong A_{n, d}$. For $d=1$ we obtain the k-algebra with generators

$$
L_{1} \rightarrow L_{2} \rightarrow L_{3} \rightarrow L_{4} \rightarrow L_{5}
$$

with no relations between the morphisms. For $d=2,3$, we obtain the k-algebras with generators

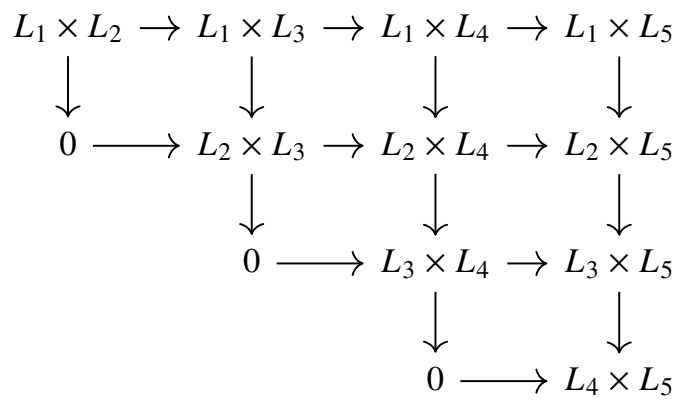

and 


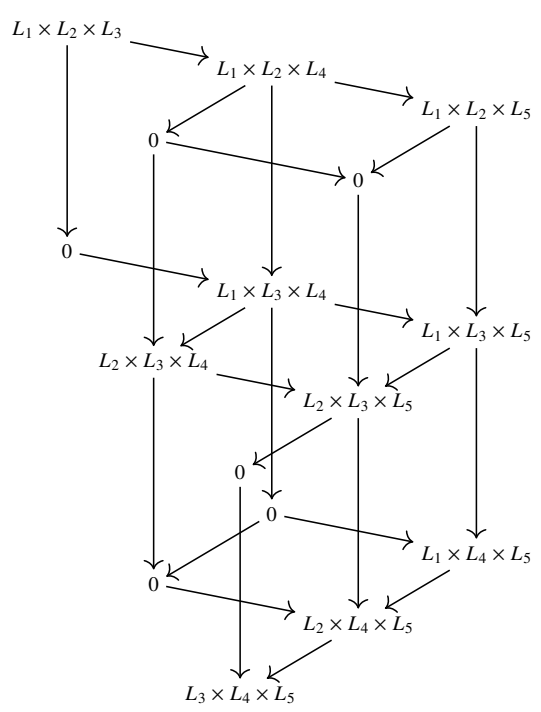

equipped with all possible commutativity relations. Finally, for $d=4$, we obtain the k-algebra with generators

$$
\prod_{i \neq 5} L_{i} \rightarrow \prod_{i \neq 4} L_{i} \rightarrow \prod_{i \neq 3} L_{i} \rightarrow \prod_{i \neq 2} L_{i} \rightarrow \prod_{i \neq 1} L_{i}
$$

and such that all consecutive composites vanish. Note that this last quiver is better drawn as a maximal path in a 4-dimensional hypercube.

We continue with the $\mathbf{k}$-algebras $\mathcal{A}_{n, d}^{\sharp} \cong A_{n, n-d}$; for simplicity, we omit the shifts on the generators $\left\{L_{I}^{\sharp}=L_{I}^{\vee}[-\mathrm{rk}(I)]\right\}$. For $d=4$, we obtain the $\mathbf{k}$-algebra with generators

$$
\prod_{i \neq 1} L_{i-1, i} \rightarrow \prod_{i \neq 2} L_{i-1, i} \rightarrow \prod_{i \neq 3} L_{i-1, i} \rightarrow \prod_{i \neq 4} L_{i-1, i} \rightarrow \prod_{i \neq 5} L_{i-1, i}
$$

and no relations between the morphisms. For $d=3,2$, we obtain the $\mathbf{k}$-algebras with generators

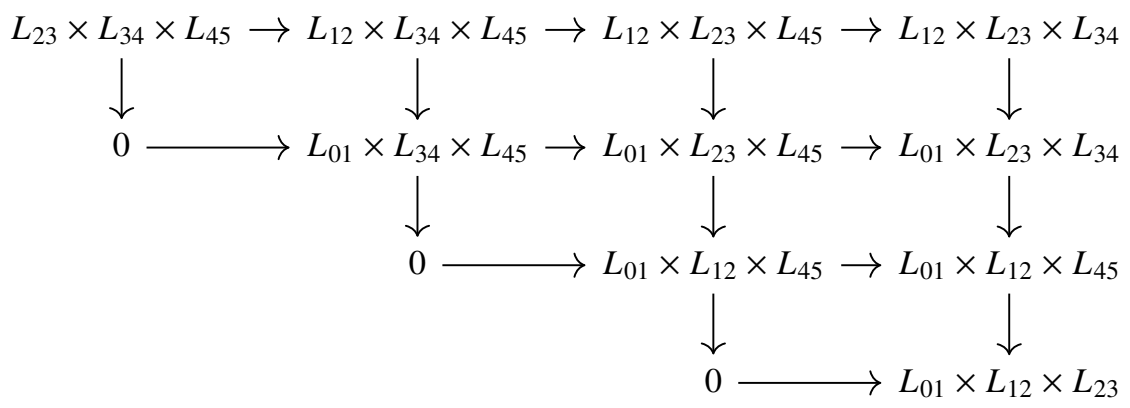

and 


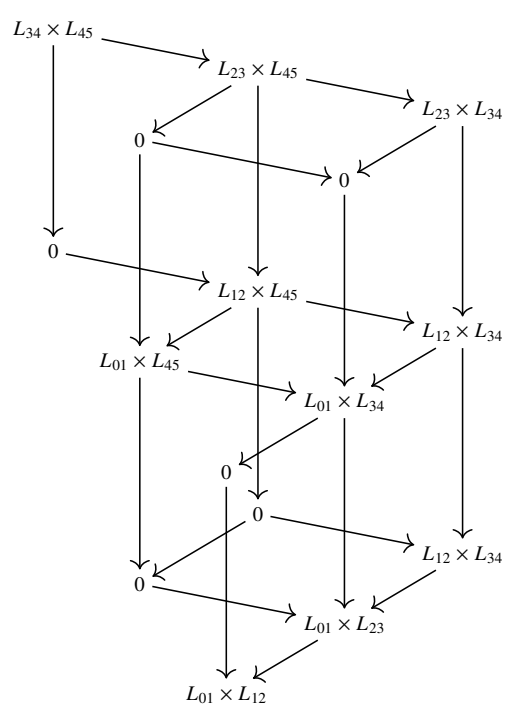

with all possible commutativity relations. Finally, for $d=1$ we obtain the $\mathbf{k}$-algebra with generators

$$
L_{45} \rightarrow L_{34} \rightarrow L_{23} \rightarrow L_{12} \rightarrow L_{01}
$$

and such that all consecutive composites vanish. Again, this last quiver is better drawn as a maximal path in a 4-dimensional hypercube.

\section{Partially wrapped Fukaya categories and models for Waldhausen $K$-theory}

In this section we provide an interpretation of the partially wrapped Fukaya categories

$$
\mathcal{W}_{n}^{(d)}=\mathcal{W}\left(\operatorname{Sym}^{d}(\mathbb{D}), \Lambda_{n}^{(d)}\right), \quad d \geq 1, n \geq 0,
$$

as the cells of a simplicial model for Waldhausen $K$-theory. This interpretation arises as an immediate consequence of the results of this work combined with the results of [DJW19], which provide a relation between the $d$-dimensional $\mathrm{S}$.-construction and the $d$-dimensional Auslander algebras of type $\mathbb{A}$. We freely use the language of $\infty$-categories [Lur09] as well as basic aspects of the theory of stable $\infty$ categories [Lur17].

\subsection{The d-dimensional Waldhausen S.-construction}

\subsubsection{Stable $\infty$-categories versus differential graded $k$-categories}

Recall that a pointed $\infty$-category $\mathcal{A}$ is stable if it admits all finite (homotopy) limits and all finite (homotopy) colimits and the suspension functor

$$
\Sigma: \mathcal{A} \longrightarrow \mathcal{A}, \quad a \longmapsto 0 \amalg_{a} 0,
$$

is an equivalence. If $\mathcal{A}$ is a stable $\infty$-category, then the homotopy category $\operatorname{Ho}(\mathcal{A})$, equipped with the suspension autoequivalence $\operatorname{Ho}(\Sigma)$, is additive and can be endowed with a canonical triangulation. For this reason, stable $\infty$-categories can be regarded as a refinement of Verdier's triangulated categories. We recall from [Coh13] that Lurie's differential graded nerve [Lur17, Construction 1.3.1.6] yields a Quillen equivalence between 
$\circ$ the homotopy theory of (small) idempotent-complete pretriangulated differential graded kcategories (up to quasi-equivalence) and

$\circ$ the homotopy theory of (small) idempotent-complete k-linear stable $\infty$-categories.

Thus, the theory of stable $\infty$-categories can be regarded as an extension of the theory of differential graded categories which encompasses higher categories that are not linear over any commutative ring, such as the stable $\infty$-category of spectra. In addition, the language of $\infty$-categories affords a powerful calculus of (homotopy) Kan extensions which which many of the statements to come are reliant upon.

In the following, we identify differential graded k-categories with their differential graded nerves without further mention. In this process, we implicitly replace $\mathcal{W}_{n}^{(d)}$ with a quasi-equivalent differential graded model (for example, by means of the differential graded Yoneda embedding; see, for example, [Sei08, Section 2(g)]).

\subsubsection{Waldhausen $K$-theory}

Notation 4.1.1. For an $\infty$-category $\mathcal{A}$, we let $\mathcal{A}^{\simeq} \subseteq \mathcal{A}$ be the largest $\infty$-groupoid (=Kan complex) contained in $\mathcal{A}$. The passage $\mathcal{A} \mapsto \mathcal{A}^{\simeq}$ yields a right adjoint to the inclusion of $\infty$-groupoids into $\infty$-categories.

An important invariant associated to a stable $\infty$-category $\mathcal{A}$ is its Waldhausen $K$-theory space $K(\mathcal{A})$. For example, if $R$ is a ring, then the Waldhausen $K$-theory space $K(R)=K(\operatorname{perf}(R))$ of the stable $\infty$-category perf $(R)$ of perfect $R$-modules is homotopy equivalent to the algebraic $K$-theory space of $R$ defined by Quillen [Qui73] in terms of the $Q$-construction of the (split-exact) category of finitely generated projective $R$-modules.

Waldhausen's definition [Wal85, BGT13] of the space $K(\mathcal{A})$ involves the construction of a simplicial $\infty$-groupoid $S_{\bullet}^{\langle 1\rangle}(\mathcal{A})^{\simeq}$ whose geometric realisation is then the delooping of the Waldhausen $K$-theory space of $\mathcal{A}$ - that is,

$$
K(\mathcal{A}):=\Omega^{1}\left|\mathrm{~S}_{\bullet}^{\langle 1\rangle}(\mathcal{A})^{\simeq}\right| .
$$

More generally, for each integer $d \geq 1$, Dyckerhoff [Dyc17b] and Poguntke [Pog17] introduce an analogous simplicial object $\mathrm{S}_{\bullet}^{\langle d\rangle}(\mathcal{A})$ such that

$$
K(\mathcal{A}) \simeq \Omega^{d}\left|\mathrm{~S}_{\bullet}^{\langle d\rangle}(\mathcal{A})^{\simeq}\right| .
$$

For each $d \geq 1$, the $d$-dimensional Waldhausen $\mathrm{S}_{\bullet}$-construction $\mathrm{S}_{\bullet}^{\langle d\rangle}(\mathcal{A})$ of $\mathcal{A}$ exhibits $K(\mathcal{A})$ as the $d$-fold loop space of a $(d-1)$-connected space or, equivalently, as a connective spectrum. We remark that the simplicial object

$$
\mathrm{S}_{\bullet}^{\langle d\rangle}(\mathcal{A}): n \longmapsto \mathrm{S}_{n}^{\langle d\rangle}(\mathcal{A})
$$

takes its values in the $\infty$-category $\mathbf{S t}_{\infty}$ of stable $\infty$-categories and exact functors between them (see [DJW19, Section 1.4] for details).

Remark 4.1.2. The $d$-dimensional Waldhausen $S_{\bullet}$-construction of a stable $\infty$-category is not the $d$-fold iteration of the $\mathrm{S}_{\bullet}$-construction, which is a multisimplicial object rather than a simplicial object.

\subsection{The equivalence $\mathcal{W}_{n}^{(d)} \simeq \mathrm{S}_{n}^{\langle d\rangle}$}

The following theorem is an immediate consequence of [DJW19, Corollary 2.25] in the case $\mathcal{A}=\operatorname{perf}(\mathbf{k})$ and our Theorem 3.2.3:

Theorem 4.2.1. Let $d \geq 1$. For each $n \geq 0$, there are equivalences of $\infty$-categories

$$
\mathrm{S}_{n}^{\langle d\rangle}(\operatorname{perf}(\mathbf{k})) \stackrel{\simeq}{\longleftarrow} \operatorname{perf}\left(A_{n, d}\right) \stackrel{\simeq}{\longrightarrow} \mathcal{W}_{n}^{(d)} .
$$


Remark 4.2.2. Let $d \geq 1$. The equivalent stable $\infty$-categories

$$
\mathrm{S}_{n}^{\langle d\rangle}(\operatorname{perf}(\mathbf{k})) \simeq \operatorname{perf}\left(A_{n, d}\right) \simeq \mathcal{W}_{n}^{(d)}
$$

vanish if $n<d$. This vanishing is closely related to the fact that the space $\left|\mathrm{S}_{\bullet}^{\langle d\rangle}(\operatorname{perf}(\mathbf{k}))^{\simeq}\right|$ is $(d-1)$ connected, which is to say that its homotopy groups vanish in degrees less than or equal to $d$.

In fact, the $d$-dimensional Waldhausen $\mathrm{S}_{\bullet}$-construction of a stable $\infty$-category can be canonically extended from a simplicial object to a paracyclic object (see [DJW19, Proposition 2.47]). In view of Theorem 4.2.1, this implies that the partially wrapped Fukaya categories $\mathcal{W}_{n}^{(d)}, n \geq 0$, themselves arrange into a paracyclic object

$$
\mathcal{W}_{\bullet}^{(d)}: \boldsymbol{\Lambda}^{\mathrm{op}} \longrightarrow \mathbf{S t}_{\infty}^{\mathbf{k}}
$$

with values in the $\infty$-category of $\mathbf{k}$-linear stable $\infty$-categories and exact functors between them, where $\boldsymbol{\Lambda}$ is the paracyclic category [Nis90, FL91, GJ93]. Passing to homotopy categories yields a paracylic object

$$
\operatorname{Ho}\left(\mathcal{W}_{\bullet}^{(d)}\right): \Lambda^{\mathrm{op}} \longrightarrow \operatorname{Ho}\left(\mathbf{S t}_{\infty}^{\mathbf{k}}\right)
$$

with values in the homotopy category of the $\infty$-category $\mathbf{S t}_{\infty}^{\mathbf{k}}$. Such a paracylic object $\operatorname{Ho}\left(\mathcal{W}_{\bullet}^{(d)}\right)$ amounts to the data of exact functors

$$
d_{0}: \mathcal{W}_{n+1}^{(d)} \longrightarrow \mathcal{W}_{n}^{(d)} \quad \text { and } \quad s_{0}: \mathcal{W}_{n}^{(d)} \longrightarrow \mathcal{W}_{n+1}^{(d)}
$$

and an (exact) autoequivalence

$$
t=t_{n+1}: \mathcal{W}_{n}^{(d)} \stackrel{\simeq}{\longrightarrow} \mathcal{W}_{n}^{(d)}
$$

for each $n \geq 0$; these functors induce additional distinguished functors

$$
d_{i}=t^{i} d_{0} t^{-i}: \mathcal{W}_{n+1}^{(d)} \longrightarrow \mathcal{W}_{n}^{(d)} \quad \text { and } \quad s_{i}=t^{i} s_{0} t^{-i}: \mathcal{W}_{n}^{(d)} \longrightarrow \mathcal{W}_{n+1}^{(d)}
$$

for each $n \geq 0$ and each $1 \leq i \leq n$. In the homotopy category $\operatorname{Ho}\left(\mathbf{S t}_{\infty}^{\mathbf{k}}\right)$, these functors must satisfy the simplicial identities

$$
\begin{array}{lll}
d_{0} s_{0}=1, & d_{0} d_{i}=d_{i-1} d_{0}, 1 \leq i \leq n, & s_{0} s_{i}=s_{i+1} s_{0}, 0 \leq i \leq n, \\
d_{1} s_{0}=1, & d_{0} s_{i}=s_{i-1} d_{0}, 1 \leq i<n, & d_{i} s_{0}=s_{0} d_{i-1}, 1<i \leq n,
\end{array}
$$

as well as the paracyclic identities

$$
d_{0} t^{n+1}=t^{n} d_{0} \quad \text { and } \quad s_{0} t^{n+1}=t^{n+2} s_{0} .
$$

By definition, these identities encode the mere existence of natural isomorphism between the corresponding functors. In contrast, the paracyclic object $\mathcal{W}_{\bullet}^{(d)}$ is a functor of $\infty$-categories, and hence it contains an infinite amount of coherence data (including explicit natural isomorphisms witnessing the validity of the identities).

Remark 4.2.3. Let $d \geq 1$. It follows from our previous discussion that there is a homotopy equivalence

$$
K\left(\mathcal{W}_{d}^{(d)}\right) \simeq K(\operatorname{perf}(\mathbf{k})) \simeq \Omega^{d}\left|\mathcal{W}_{\bullet}^{(d)}\right|,
$$


where we remind the reader that $\mathcal{W}_{d}^{(d)}$ is equivalent to perf $(\mathbf{k})$. More generally, if $(M, \Lambda)$ is an arbitrary Weinstein manifold with stops, there are equivalences of stable $\infty$-categories

$$
\begin{aligned}
\mathcal{W}\left(M \times \operatorname{Sym}^{d}(\mathbb{D}),\left(\Lambda \times \operatorname{Sym}^{d}(\mathbb{D})\right) \cup\left(M \times \Lambda_{n}^{(d)}\right)\right) & \simeq \operatorname{Fun}_{\mathbf{k}}\left(\mathcal{W}_{n}^{(d)}, \mathcal{W}(M, \Lambda)\right) \\
& \simeq \operatorname{Fun}_{\mathbf{k}}\left(A_{n, d}, \mathcal{W}(M, \Lambda)\right) \\
& \simeq \mathrm{S}_{n}^{\langle d\rangle}(\mathcal{W}(M, \Lambda)) .
\end{aligned}
$$

Indeed:

$\circ$ the leftmost equivalence is a consequence of

- the Künneth formula [GPS18, Corollary 1.11]

$$
\begin{gathered}
\mathcal{W}(M, \Lambda) \otimes_{\mathbf{k}} \mathcal{W}\left(\operatorname{Sym}^{d}(\mathbb{D}), \Lambda_{n}^{(d)}\right) \\
\downarrow \sim \sim \\
\mathcal{W}\left(M \times \operatorname{Sym}^{d}(\mathbb{D}),\left(\Lambda \times \operatorname{Sym}^{d}(\mathbb{D})\right) \cup\left(M \times \Lambda_{n}^{(d)}\right)\right),
\end{gathered}
$$

- the equivalence

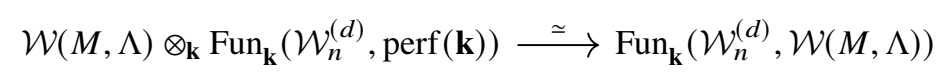

- and the equivalences

$$
\operatorname{Fun}_{\mathbf{k}}\left(\mathcal{W}_{n}^{(d)}, \operatorname{perf}(\mathbf{k})\right) \simeq\left(\mathcal{W}_{n}^{(d)}\right)^{\mathrm{op}} \simeq \mathcal{W}_{n}^{(d)}=\mathcal{W}\left(\operatorname{Sym}^{d}(\mathbb{D}), \Lambda_{n}^{(d)}\right),
$$

where the existence of the second and third equivalences is a consequence of the fact that $\mathcal{W}_{n}^{(d)} \simeq$ $\operatorname{perf}\left(A_{n, d}\right)$ is a dualisable object of the symmetric monoidal $\infty$-category $\left(\mathbf{S t}_{\infty}^{\mathbf{k}}, \otimes_{\mathbf{k}}, \operatorname{perf}(\mathbf{k})\right)$, since the higher Auslander algebra $A_{n, d}$ is proper and homologically smooth over k;

○ the middle equivalence stems from the fact that $\mathcal{W}_{n}^{(d)} \simeq \operatorname{perf}\left(A_{n, d}\right)$ is the $\mathbf{k}$-linear stable hull of the k-algebra $A_{n, d}$; and

$\circ$ the rightmost equivalence is a consequence of [DJW19, Propositions 2.10 and 2.24].

Consequently, we have a paracyclic object

$$
\mathcal{W}\left(M \times \operatorname{Sym}^{d}(\mathbb{D}),\left(\Lambda \times \operatorname{Sym}^{d}(\mathbb{D})\right) \cup\left(M \times \Lambda_{\bullet}^{(d)}\right)\right): \Lambda^{\mathrm{op}} \longrightarrow \mathbf{S t}_{\infty}^{\mathbf{k}}
$$

and homotopy equivalences

$$
\begin{aligned}
K(\mathcal{W}(M, \Lambda)) & \simeq \Omega^{d}\left|\mathrm{~S}_{\bullet}^{\langle d\rangle}(\mathcal{W}(M, \Lambda))^{\simeq}\right| \\
& \simeq \Omega^{d}\left|\mathcal{W}\left(M \times \operatorname{Sym}^{d}(\mathbb{D}),\left(\Lambda \times \operatorname{Sym}^{d}(\mathbb{D})\right) \cup\left(M \times \Lambda_{\bullet}^{(d)}\right)\right)^{\simeq}\right|
\end{aligned}
$$

which describe the $d$-fold delooping of the Waldhausen $K$-theory space of the partially wrapped Fukaya category $\mathcal{W}(M, \Lambda)$ by means of partially wrapped Fukaya categories of symmetric products of marked disks. Compare with [Tan19, Section 1.2], where the case $d=1$ is discussed.

4.2.1. Symplecto-geometric description of the structure maps of $\mathcal{W}_{\bullet}^{(d)}$

The generating structure maps of the paracyclic object $\mathcal{W}_{\bullet}^{(d)}$ admit a natural symplecto-geometric interpretation. Fix $n \geq 0$. First, the paracyclic shift

$$
t: \mathcal{W}_{n}^{(d)} \simeq \mathcal{W}_{n}^{(d)}
$$


can be identified with the autoequivalence $\mathfrak{r}^{-1}[d]$ of $\mathcal{W}_{n}^{(d)}$, which, according to Proposition 3.5.1, is induced by rotating the disk clockwise by an angle of $\frac{2 \pi}{n+1}$ (here $\mathfrak{r}$ denotes the Serre functor of $\left.\mathcal{W}_{n}^{(d)}\right)$.

Second, for $0 \leq i \leq n+1$, the face functor

$$
d_{i}: \mathcal{W}_{n+1}^{(d)} \stackrel{\simeq}{\longrightarrow} \mathcal{W}_{n}^{(d)}
$$

can be identified with the stop-removal functor which removes the stop $\left\{p_{i}\right\} \times \operatorname{Sym}^{d-1}(\mathbb{D})$ from $\Lambda_{n+1}^{(d)}$. These functors are described as follows: consider the smallest idempotent-complete stable subcategory of $\mathcal{W}_{n+1}^{(d)}$ containing the objects of the form

$$
L \times L_{i-1, i}
$$

where $L$ is a product of $d-1$ mutually disjoint $\operatorname{arcs}$ in $\mathbb{D} \backslash \Lambda_{n}^{(d)}$ which are also disjoint from the arc $L_{i-1, i}$; here, we identify $\Lambda_{n}$ with $\Lambda_{n+1} \backslash\left\{p_{i}\right\}$. Equivalently, this subcategory is the essential image of the Orlov functor

$$
\iota_{i}: \mathcal{W}_{n}^{(d-1)} \longrightarrow \mathcal{W}_{n+1}^{(d)}, \quad X \longmapsto X \times L_{i-1, i}
$$

The functor $d_{i}$ is defined in terms of the Verdier quotient ${ }^{1}$

$$
\mathcal{W}_{n}^{(d-1)} \stackrel{\iota_{i}}{\longrightarrow} \mathcal{W}_{n+1}^{(d)} \stackrel{d_{i}}{\longrightarrow} \mathcal{W}_{n+1}^{(d)} / \mathcal{W}_{n}^{(d-1)}
$$

of $\mathcal{W}_{n+1}^{(d)}$ by the essential image of $\iota_{i}$. As a consequence of general stop-removal theorems [GPS18, Syl19a, Syl19b], we can identify the target $\mathcal{W}_{n+1}^{(d)} / \mathcal{W}_{n}^{(d-1)}$ of the localisation functor $d_{i}$ with the partially wrapped Fukaya category $\mathcal{W}_{n}^{(d)}$.

Let $1 \leq i \leq n$. Finally, we describe the degeneracy functor

$$
s_{i}: \mathcal{W}_{n}^{(d)} \longrightarrow \mathcal{W}_{n+1}^{(d)}
$$

For this, we introduce a new stop into $\Lambda_{n}^{(d)}$ by adding positive push-off of the component $\left\{p_{i}\right\} \times$ $\operatorname{Sym}^{d-1}(\mathbb{D})$ in the direction of the Reeb flow on the disk. That is, we consider the new set of stops

$$
\Lambda_{n+1}^{(d)}=\Lambda_{n}^{(d)} \cup\left\{\left\{p_{i}^{\varepsilon}\right\} \times \operatorname{Sym}^{d-1}(\mathbb{D})\right\},
$$

where $p_{i}^{\varepsilon}$ is a point on the boundary of the disk obtained by rotating $p_{i}$ by a small angle $\varepsilon>0$ in the counterclockwise direction. This construction gives rise to a push-forward functor $s_{i}$ with the desired source and target categories. The restriction of the functor $s_{i}$ to the full subcategory of $\mathcal{W}_{n}^{(d)}$ spanned by those objects given by products of disjoint $\operatorname{arcs}$ in $\mathbb{D} \backslash \Lambda_{n}$ is the identity functor, from which it readily follows that $s_{i}$ is fully faithful. This is a consequence of the fact that at the geometric level, a product of $\operatorname{arcs}$ in $\mathbb{D} \backslash \Lambda_{n}$ can also be seen as a product of $\operatorname{arcs}$ in $\mathbb{D} \backslash\left(\Lambda_{n} \cup\left\{p_{i}^{\varepsilon}\right\}\right)$; as can be seen from Auroux's description of morphisms in terms of strand diagrams, the fact that $\varepsilon$ is chosen to be sufficiently small guarantees that $s_{i}$ induces an isomorphism between the cochain complexes of morphisms between such objects. The construction of the functor $s_{i}$ should be compared with the forward stopped inclusions introduced in [GPS18].

\footnotetext{
${ }^{1}$ Under the differential graded nerve, the Verdier quotient of $\mathbf{k}$-linear stable $\infty$-categories corresponds to the Drinfeld quotient [Dri04] of differential graded k-categories. This is a consequence of the fact that both quotients are characterised as homotopy cofibres in the corresponding $\infty$-categories.
} 


\subsubsection{Relation to the categorified Dold-Kan correspondence}

As a consequence of a categorified version of the Dold-Kan correspondence [Dyc17b], the $d$ dimensional Waldhausen $S_{\bullet}$-construction of $\operatorname{perf}(\mathbf{k})$ is characterised, as a 2-simplicial object, by the existence of equivalences of stable $\infty$-categories $\mathrm{S}_{0}^{\langle d\rangle}(\mathcal{A}) \simeq 0$ and

$$
\bigcap_{i=1}^{n} \operatorname{ker}\left(d_{i}: \mathrm{S}_{n}^{\langle d\rangle}(\mathcal{A}) \rightarrow \mathrm{S}_{n-1}^{\langle d\rangle}(\mathcal{A})\right) \simeq \begin{cases}\operatorname{perf}(\mathbf{k}) & \text { if } n=d, \\ 0 & \text { otherwise }\end{cases}
$$

for $n \geq 1$. These vanishing conditions have a natural interpretation in terms of the stop-removal functors already described.

Proposition 4.2.4. Let $d \geq 1$. There are equivalences of stable $\infty$-categories $\mathcal{W}_{d}^{(0)} \simeq 0$ and

$$
\overline{\mathcal{W}}_{n}^{(d)}:=\bigcap_{i=1}^{n} \operatorname{ker}\left(d_{i}: \mathcal{W}_{n}^{(d)} \rightarrow \mathcal{W}_{n-1}^{(d)}\right) \simeq \begin{cases}\operatorname{perf}(\mathbf{k}) & \text { if } n=d, \\ 0 & \text { otherwise }\end{cases}
$$

for $n \geq 1$.

Proof. The claim is obvious for $n<d$, since $\mathcal{W}_{n}^{(d)}$ vanishes in this case. For $n=d$ there is an equivalence

$$
\operatorname{perf}(\mathbf{k}) \simeq \mathcal{W}_{d}^{(d)}=\overline{\mathcal{W}}_{n}^{(d)}=\bigcap_{i=1}^{d} \operatorname{ker}\left(d_{i}: \mathcal{W}_{d}^{(d)} \rightarrow \mathcal{W}_{d-1}^{(d)}\right),
$$

since $\mathcal{W}_{d-1}^{(d)} \simeq 0$ and the higher Auslander algebra $A_{d, d}$ is isomorphic to the base commutative ring $\mathbf{k}$ in this case. It remains to prove that the intersection

$$
\overline{\mathcal{W}}_{n}^{(d)}=\bigcap_{i=1}^{n} \operatorname{ker}\left(d_{i}: \mathcal{W}_{n}^{(d)} \rightarrow \mathcal{W}_{n-1}^{(d)}\right)=\bigcap_{i=1}^{n} \operatorname{im}\left(\iota_{i}: \mathcal{W}_{n-1}^{(d-1)} \hookrightarrow \mathcal{W}_{n}^{(d)}\right)
$$

vanishes for $n>d$, where $\iota_{i}$ is the Orlov functor corresponding to the point $p_{i} \in \Lambda_{n}$. This is clear because $\overline{\mathcal{W}}_{n}^{(d)}$ is generated by Lagrangians of the form $\prod_{i=1}^{d} L_{i}$, where $L_{1}, \ldots, L_{d}$ are pairwise nonintersecting $\operatorname{arcs}$ in $\mathbb{D} \backslash \Lambda_{n}$ that must be jointly supported near all the stops $p_{1}, \ldots, p_{n}$; but the assumption that $n>d$ implies that no such a collection of arcs exists. The claim follows.

Remark 4.2.5. An interesting challenge - which we do not pursue here - is to extend these descriptions to a construction of the paracyclic object

$$
\mathcal{W}_{\bullet}^{(d)}: \Lambda^{\mathrm{op}} \longrightarrow \mathbf{S t}_{\infty}^{\mathbf{k}}
$$

carried entirely within the framework of partially wrapped Fukaya categories (see [Tan19] for a related discussion in the case $d=1$ ). In particular, notice that we have not described the higher-order components of the paracyclic object $\mathcal{W}_{\bullet}^{(d)}$, nor have we given symplectic explanations of the fact that these satisfy the required coherence equations.

Acknowledgments. The authors thank Th. Kragh, N. Williams and an anonymous referee for comments on an earlier version of this article. The third author would like to thank D. Nadler for motivating conversations around this topic.

\section{Conflict of Interest: None.}

Financial support. The first author acknowledges the support of the VolkswagenStiftung through the Lichtenberg Professorship Programme. His research is further supported by the Deutsche Forschungsgemeinschaft (German Research Foundation) under Germany's Excellence Strategy - EXC 2121 'Quantum Universe' - 390833306. The second author was funded by the Deutsche Forschungsgemeinschaft (German Research Foundation) under Germany's Excellence Strategy - GZ 2047/1, ProjektID 390685813 . The third author was partially funded by the Royal Society URF\R\180024. 


\section{References}

[APS19] C. Amiot, P.-G. Plamondon and S. Schroll, 'A complete derived invariant for gentle algebras via winding numbers and arf invariants', Preprint, 2019, arXiv:1904.02555.

[AS10] M. Abouzaid and P. Seidel, 'An open string analogue of Viterbo functoriality', Geom. Topol. 14(2) (2010), 627-718.

[Aur10a] D. Auroux, 'Fukaya categories and bordered Heegaard-Floer homology', in Proceedings of the International Congress of Mathematicians, Vol. II, (Hindustan Book Agency, New Delhi, 2010), 917-941.

[Aur10b] D. Auroux, 'Fukaya categories of symmetric products and bordered Heegaard-Floer homology', J. Gökova Geom. Topol. GGT 4 (2010), 1-54.

[Aus71] M. Auslander, 'Representation Dimension of Artin Algebras' Queen Mary College Math. Notes, Queen Mary College, London (1971) reprinted in: Selected Works of Maurice Auslander, part 1, edited and with a foreword by Idun Reiten, Sverre O. Smalø, and Ø yvind Solberg, Amer. Math. Soc., Providence, 1999

[BB05] A. Björner and F. Brenti, Combinatorics of Coxeter Groups, Graduate Texts in Mathematics, 231 (Springer, New York, 2005).

[Bec18] F. Beckert, The Bivariant Parasimplicial S•-construction, Ph.D. thesis, Bergische Universität Wuppertal, 2018.

[BGT13] A. J. Blumberg, D. Gepner and G. Tabuada, 'A universal characterization of higher algebraic K-theory', Geom. Topol. 17(2) (2013), 733-838.

[Boc16] R. Bocklandt, 'Noncommutative mirror symmetry for punctured surfaces', Trans. Amer. Math. Soc. 368(1) (2016), 429-469. With an appendix by M. Abouzaid.

[Coh13] L. Cohn, 'Differential graded categories are $k$-linear stable infinity categories'. Preprint, 2013, arXiv:1308.2587.

[DJW19] T. Dyckerhoff, G. Jasso and T. Walde, 'Simplicial structures in higher Auslander-Reiten theory', Adv. Math. 355 (2019), 106762.

[DK18] T. Dyckerhoff and M. Kapranov, 'Triangulated surfaces in triangulated categories', J. Eur. Math. Soc. (JEMS) 20(6) (2018), 1473-1524.

[DLRS10] J. A. De Loera, J. Rambau and F. Santos, Triangulations: Structures for Algorithms and Applications, Algorithms and Computation in Mathematics, 25 (Springer-Verlag, Berlin, 2010).

[Dri04] V. Drinfeld, 'DG quotients of DG categories', J. Algebra 272(2) (2004), 643-691.

[Dyc17a] T. Dyckerhoff, ' $A$-homotopy invariants of topological Fukaya categories of surfaces', Compos. Math. 153(8) (2017), 1673-1705.

[Dyc17b] T. Dyckerhoff, 'A categorified Dold-Kan correspondence'. Preprint, 2017, arXiv:1710.08356.

[FL91] Z. Fiedorowicz and J.-L. Loday, 'Crossed simplicial groups and their associated homology', Trans. Amer. Math. Soc. 326(1) (1991), 57-87.

[GI19] J. Grant and O. Iyama, 'Higher preprojective algebras, Koszul algebras, and superpotentials', Compos. Math., to appear. Preprint, 2019, arXiv:1902.07878.

[GJ93] E. Getzler and J. D. S. Jones, 'The cyclic homology of crossed product algebras', J. Reine Angew. Math. 445 (1993), 161-174.

[GKO13] C. Geiss, B. Keller and S. Oppermann, ' $n$-angulated categories', J. Reine Angew. Math. 675 (2013), 101-120.

[GPS18] S. Ganatra, J. Pardon and V. Shende, 'Structural results in wrapped Floer theory', Preprint, 2018, arXiv:1809.03427.

[GPS19] S. Ganatra, J. Pardon and V. Shende, 'Covariantly functorial wrapped Floer theory on Liouville sectors', Publications mathématiques de l'IHÉS 131 (2020), 73-200.

[Hap88] D. Happel, Triangulated Categories in the Representation Theory of Finite-Dimensional Algebras, London Mathematical Society Lecture Note Series, 119 (Cambridge University Press, Cambridge, 1988).

[HI11] M. Herschend and O. Iyama, ' $n$-representation-finite algebras and twisted fractionally Calabi-Yau algebras', Bull. Lond. Math. Soc. 43(3) (2011), 449-466.

[HIO14] M. Herschend, O. Iyama and S. Oppermann, ' $n$-representation infinite algebras', Adv. Math. 252 (2014), $292-342$.

[HKK17] F. Haiden, L. Katzarkov and M. Kontsevich, 'Flat surfaces and stability structures', Publ. Math. Inst. Hautes Études Sci. 126(1) (2017), 247-318.

[IJ17] O. Iyama and G. Jasso, 'Higher Auslander correspondence for dualizing R-varieties', Algebr. Represent. Theory 20(2) (2017), 335-354.

[IO11] O. Iyama and S. Oppermann, ' $n$-representation-finite algebras and $n$-APR tilting', Trans. Amer. Math. Soc. 363(12) (2011), 6575-6614.

[IO13] O. Iyama and S. Oppermann, 'Stable categories of higher preprojective algebras', Adv. Math. 244 (2013), 23-68.

[Iya07a] O. Iyama, 'Auslander correspondence', Adv. Math. 210(1) (2007), 51-82.

[Iya07b] O. Iyama, 'Higher-dimensional Auslander-Reiten theory on maximal orthogonal subcategories', Adv. Math. 210(1) (2007), 22-50.

[Iya11] O. Iyama, 'Cluster tilting for higher Auslander algebras', Adv. Math. 226(1) (2011), 1-61.

[Jas16] G. Jasso, ' $n$-abelian and $n$-exact categories', Math. Z. 283(3-4) (2016), 703-759.

[JK19] G. Jasso and J. Külshammer, 'Higher Nakayama algebras I: Construction', Adv. Math. 351 (2019), 1139-1200. With an appendix by J. Külshammer and Ch. Psaroudakis and an appendix by S. Kvamme.

[Kel94] B. Keller, 'Deriving DG categories', Ann. Sci. Éc. Norm. Supér. (4) 27(1) (1994), 63-102. 
[Kel06] B. Keller, 'On differential graded categories', in International Congress of Mathematicians, Vol. II, (European Mathematical Society, Zürich, 2006), 151-190.

[Kho14] M. Khovanov, 'How to categorify one-half of quantum gl (1|2)', in Knots in Poland III. Part III, Banach Center Publications, 103 (Institute of Mathematics of the Polish Academy of Sciences, Warsaw, 2014), 211-232.

[Kon09] M. Kontsevich, 'Symplectic geometry of homological algebra' (2009). URL: http://www.ihes.fr/ maxim/TEXTS/Symplectic_AT2009.pdf.

[LOT15] R. Lipshitz, P. S. Ozsváth and D. P. Thurston, 'Bimodules in bordered Heegaard Floer homology', Geom. Topol. 19(2) (2015), 525-724.

[LOT18] R. Lipshitz, P. S. Ozsváth and D. P. Thurston, 'Bordered Heegaard Floer homology', Mem. Amer. Math. Soc. 254(1216) (2018), viii+279.

[LP18] Y. Lekili and A. Polishchuk, 'Auslander orders over nodal stacky curves and partially wrapped Fukaya categories', $J$. Topol. 11(3) (2018), 615-644.

[LP20a] Y. Lekili and A. Polishchuk, 'Derived equivalences of gentle algebras via Fukaya categories', Math. Ann. 376(1-2) (2020), 187-225.

[LP20b] Y. Lekili and A. Polishchuk, 'Homological mirror symmetry for higher-dimensional pairs of pants', Compos. Math. 156(7) (2020), 1310-1347.

[LS14] V. A. Lunts and O. M. Schnürer, 'Smoothness of equivariant derived categories', Proc. Lond. Math. Soc. (3) 108(5) (2014), 1226-1276.

[Lur09] J. Lurie, Higher Topos Theory, Annals of Mathematics Studies, 170 (Princeton University Press, Princeton, NJ, 2009).

[Lur17] J. Lurie, 'Higher algebra' (May 2017). URL: http://www.math.harvard.edu/ lurie/.

[Miy86] Y. Miyashita, 'Tilting modules of finite projective dimension', Math. Z. 193(1) (1986), 113-146.

[Nis90] V. Nistor, 'Group cohomology and the cyclic cohomology of crossed products', Invent. Math. 99(2) (1990), 411-424.

[Opp19] S. Opper, 'On auto-equivalences and complete derived invariants of gentle algebras', Preprint, 2019, arXiv:1904.04859.

[OT12] S. Oppermann and H. Thomas, 'Higher-dimensional cluster combinatorics and representation theory', J. Eur. Math. Soc. (JEMS) 14(6) (2012), 1679-1737.

[Per08] T. Perutz, 'Hamiltonian handleslides for Heegaard Floer homology', in Proceedings of Gökova Geometry-Topology Conference 2007 (International Press, Gökova, 2008), 15-35.

[Pog17] T. Poguntke, 'Higher Segal structures in algebraic $K$-theory', Preprint, 2017, arXiv:1709.06510.

[Qui73] D. Quillen, 'Higher algebraic K-theory. I', Lecture Notes in Math. 341 (1973), 85-147.

[Ram97] J. Rambau, 'Triangulations of cyclic polytopes and higher Bruhat orders', Mathematika 44(1) (1997), $162-194$.

[Sei00] P. Seidel, 'Graded Lagrangian submanifolds', Bull. Soc. Math. France 128(1) (2000), 103-149.

[Sei08] P. Seidel, 'Fukaya categories and Picard-Lefschetz theory', Zurich Lectures in Advanced Mathematics (European Mathematical Society, Zürich, 2008).

[Syl19a] Z. Sylvan, 'On partially wrapped Fukaya categories', J. Topol. 12(2) (2019), 372-441.

[Syl19b] Z. Sylvan, 'Orlov and Viterbo functors in partially wrapped Fukaya categories', Preprint, 2019, arXiv:1908.02317.

[Tac64] H. Tachikawa, 'On dominant dimensions of QF-3 algebras', Trans. Amer. Math. Soc. 112 (1964), $249-266$.

[Tan19] H. L. Tanaka, 'Cyclic structures and broken cycles', Preprint, 2019, arXiv:1907.03301.

[TV07] B. Toën and M. Vaquié, 'Moduli of objects in dg-categories', Ann. Sci. Éc. Norm. Supér. (4) 40(3) (2007), $387-444$.

[Wal85] F. Waldhausen, 'Algebraic K-theory of spaces', in Algebraic and Geometric Topology (New Brunswick, N.J., 1983), Lecture Notes in Math., 1126 (Springer, Berlin, 1985), 318-419. 